\title{
Trends in particle and nuclei identification techniques in nuclear physics experiments
}
A. Badalà ${ }^{1}$ - M. La Cognata ${ }^{2} \cdot$ R. Nania ${ }^{3}$ - M. Osipenko ${ }^{4} \cdot$ S. Piantelli ${ }^{5}$.
R. Turrisi ${ }^{6}$. L. Barion ${ }^{7}$. S. Capra ${ }^{8,9}$ - D. Carbone ${ }^{2}$. F. Carnesecchi ${ }^{3,26}$.
E. A. R. Casula ${ }^{11,12}$. C. Chatterjee ${ }^{13}$. G. F. Ciani ${ }^{14,25} \cdot$ R. Depalo ${ }^{6,15}$.

A. Di Nitto ${ }^{16,17}$ - A. Fantini ${ }^{18,27}$ - A. Goasduff ${ }^{19}$ - G. L. Guardo ${ }^{2}$ A. C. Kraan ${ }^{20}$. A. Manna ${ }^{3,10}$ - L. Marsicano ${ }^{4}$. N. S. Martorana 2,21 . L. Morales-Gallegos ${ }^{16}$. E. Naselli, ${ }^{2,21} \cdot$ A. Scordo 22 . S. Valdré5 $\cdot$ G. Volpe 23,24

Received: 1 June 2021 / Accepted: 7 October 2021 / Published online: 8 March 2022

(c) The Author(s) 2022, corrected publication 2022

\begin{abstract}
Particle identification techniques are fundamental tools in nuclear physics experiments. Discriminating particles or nuclei produced in nuclear interactions allows to better understand the underlying physics mechanisms. The energy interval of these reactions is very broad, from sub-eV up to TeV. For this reason, many different identification approaches have been developed, often combining two or more observables. This paper reviews several of these techniques with emphasis on the expertise gained within the current nuclear physics scientific program of the Italian Istituto Nazionale di Fisica Nucleare (INFN).
\end{abstract}

Keywords Nuclear physics experiments $\cdot$ Particle identification $\cdot$ Nuclei identification · Gamma identification · PSA · TOF · Calorimetry · DE-E · RICH

\section{Contents}

1 Introduction . . . . . . . . . . . . . . . . . . . . . . . . . 191

2 Nuclear physics experiments in CSN3 implementing PID techniques . . . . . . . . . . . . . . 192

2.1 Quark and hadron dynamics . . . . . . . . . . . . . . . . . . . . . . 192

2.1.1 EIC: electron ion collider at Brookhaven National Laboratory (BNL) . . . . . . . . . 192

2.1.2 JLAB: studies on hadron structure at Jefferson Lab . . . . . . . . . . . . . . . . . 193

2.1.3 KAONNIS: studies of Kaonic atoms at DAФNE LNF . . . . . . . . . . . . . . . . 193

2.1.4 MAMBO: meson photoproduction on nucleon in the BGOOD experiment . . . . . . . 194

2.2 Phase transition of nuclear and hadronic matter . . . . . . . . . . . . . . . . . . . . . . 194

S. Piantelli

silvia.piantelli@fi.infn.it

$凶 \quad$ R. Turrisi

rosario.turrisi@pd.infn.it

Extended author information available on the last page of the article 
2.2.1 ALICE: QCD studies in extreme conditions with heavy-ion collisions at the CERN LHC . . . . . 194

2.3 Nuclear structure and reaction dynamics . . . . . . . . . . . . . . . . . . . . . . . 195

2.3.1 CHIRONE: heavy-ion nuclear reactions in a wide range of energies . . . . . . . . . 195

2.3.2 FORTE: dynamics of fission and shell effects in super-heavy elements . . . . . . . 196

2.3.3 GAMMA: study of the nuclear structure using $\gamma$ spectroscopy . . . . . . . . . . . . . . 196

2.3.4 NUCLEX: nuclear dynamics and thermodynamics with heavy ions . . . . . . . . . . . 197

2.3.5 NUMEN: nuclear matrix elements of neutrinoless double beta decays . . . . . . . . . 197

2.3.6 PRISMA: heavy-ions dynamics with a large angle magnetic spectrometer at LNL . . . 198

2.4 Nuclear astrophysics . . . . . . . . . . . . . . . . . . . . . . . . . . . . . . . . . . 199

2.4.1 ASFIN: nuclear astrophysics studies at very low energy . . . . . . . . . . . . . . . 199

2.4.2 ERNA: direct measurements for $\mathrm{C}$ burning in stars . . . . . . . . . . . . . . . . . 199

2.4.3 LUNA: study of nuclear reactions for astrophysics at LNGS . . . . . . . . . . . . . 200

2.4 .4 n_TOF: neutron time of flight facility at CERN . . . . . . . . . . . . . . . . 200

2.4.5 PANDORA: plasma for astrophysics, nuclear decays observation and radiation for

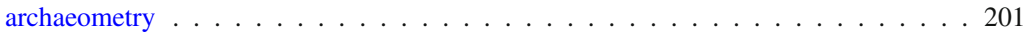

2.5 Applications and societal benefits . . . . . . . . . . . . . . . . . . . 201

2.5.1 FOOT: nuclear cross section measurements for hadron therapy . . . . . . . . . . 201

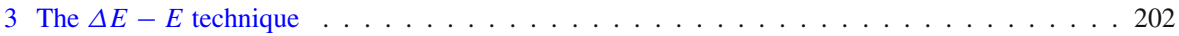

3.1 The low-energy limit: research in nuclear astrophysics . . . . . . . . . . . . . . . . 203

3.1.1 ERNA: advanced ionization chamber as $\Delta E$ detectors . . . . . . . . . . . . . . 203

3.1.2 ASFIN: pushing the low-thickness limit of solid-state devices . . . . . . . . . . . . . 204

3.2 Nuclear structure and dynamics experiments at intermediate energies . . . . . . . . . . . . 206

3.2.1 CHIRONE: energy loss in large acceptance arrays . . . . . . . . . . . . . . . . . 206

3.2 .2 NUCLEX: tailored $\Delta E-E$ solutions . . . . . . . . . . . . . . . . . . . . 207

3.2 .3 NUMEN: combining $\Delta E$ and magnetic rigidity measurement . . . . . . . . . . 209

3.3 ALICE: the high-energy limit for particles and light nuclei at the GeV scale . . . . . . . . . 210

3.4 FOOT: particle identification for light fragment cross section measurements with emulsions . . . . 212

4 Pulse shape analysis (PSA) for charged particles, neutrons and $\gamma$-rays . . . . . . . . . . . . . 212

4.1 PSA technique in $\mathrm{CsI}(\mathrm{Tl})$ scintillators . . . . . . . . . . . . . . . . . . . 213

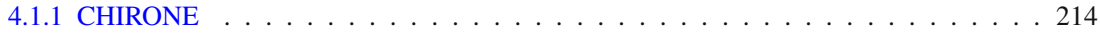

4.1 .2 NUCLEX . . . . . . . . . . . . . . . . . . . . . . . . . 214

4.2 PSA in silicon detectors . . . . . . . . . . . . . . . . . . . . . 216

4.2 .1 CHIRONE . . . . . . . . . . . . . . . . . . . . 216

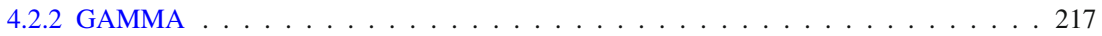

4.2 .3 NUCLEX . . . . . . . . . . . . . . . . . . . . . . . . 218

$4.3 \mathrm{n}-\gamma$-Charged particles and/or $\mathrm{n}-\gamma$ discrimination . . . . . . . . . . . . . . 220

4.3 .1 CHIRONE . . . . . . . . . . . . . . . . . . . 220

4.3 .2 GAMMA . . . . . . . . . . . . . . . . . . . 221

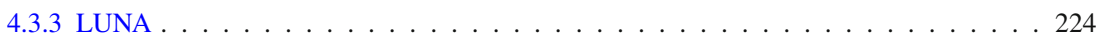

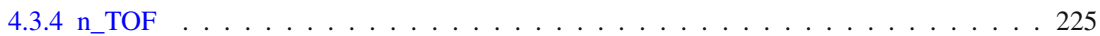

4.3 .5 NUCLEX . . . . . . . . . . . . . . . . . . . . . . . . . . . . . . . . . . . . . . . . . . . . . . . . . . .

$4.4 \gamma$-ray tracking with HPGe detectors . . . . . . . . . . . . . . . . . . . . . 228

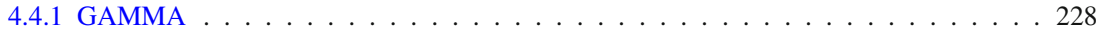

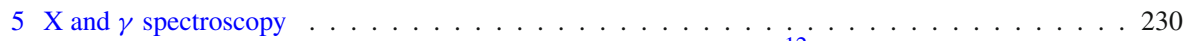

5.1 CHIRONE: identification of Pygmy Dipole Resonance and of ${ }^{12} \mathrm{C}$ excited states with

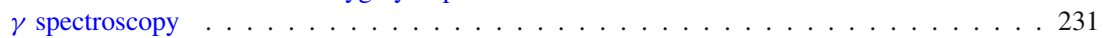

5.2 KAONNIS: kaonic atom identification by X-ray spectroscopy . . . . . . . . . . . . . . . . 232

5.3 PANDORA: ion identification with X-ray spectroscopy and isotope identification with

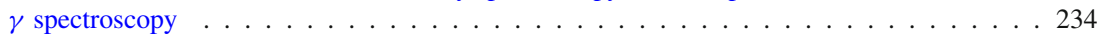

6 Particle identification by means of time of flight combined with energy or magnetic field analysis . . . 235

6.1 Particle and very light nuclei identification in the $\approx 100 \mathrm{MeV}$ to $\mathrm{GeV}$ energy range . . . . . . 235

6.1.1 ALICE TOF: MRPCs for particles up to $5 \mathrm{GeV} / c \ldots \ldots$. . . . . . . . . . . . . . 235

6.1 .2 KAONNIS: kaon identification at $D A \Phi N E$-LNF . . . . . . . . . . . . . . . . 237

6.1 .3 n_TOF: identification of neutron-induced elastic reactions . . . . . . . . . . . . 238

6.2 Nuclei identification in energy range of the order of $10 \mathrm{MeV} /$ nucleon . . . . . . . . . . . 239

6.2.1 ASFIN: $\alpha$ identification to define elastic and inelastic interactions . . . . . . . . . . . 239

6.2.2 CHIRONE: light and heavy nuclei identification with silicon detectors . . . . . . . . . 241 
6.2.3 FOOT: particle identification for heavy fragment cross section measurements with $E$ TOF method . . . . . . . . . . . . . . . . . . . . . 242

6.2.4 FORTE: separation of fusion-fission and quasi-elastic events . . . . . . . . . . . . . . . 244

6.2.5 NUCLEX: identification of charged products fragments with silicon detectors . . . . . 245

6.2.6 PRISMA: heavy nuclei identification with a magnetic spectrometer . . . . . . . . . . 246

7 Calorimeters and muon identification . . . . . . . . . . . . . . . . . . . . . . . . 247

7.1 ALICE: electrons, photons and muons tagging in a high-multiplicity, high-energy environment . . 248

7.1.1 Electromagnetic calorimeter . . . . . . . . . . . . . . . . . . . 248

7.1 .2 Muon spectrometer . . . . . . . . . . . . . . . . . . . . . . . . . . . 249

7.2 JLAB: electron-photon discrimination at an electron beam facility . . . . . . . . . . . . 250

7.3 BGOOD (MAMBO): neutral particles discrimination with BGO . . . . . . . . . . . . 252

8 Ring-imaging Cherenkov detectors . . . . . . . . . . . . . . . . . . . . 253

8.1 ALICE: the largest CsI RICH application for pion, kaon, proton separation . . . . . . . . . . 254

8.2 EIC: perspective of PID techniques at the electron-ion collider . . . . . . . . . . . . 256

8.3 JLAB: advanced compact and optimised design of RICH at CEBAF . . . . . . . . . . . 258

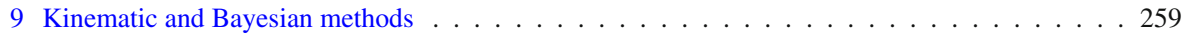

9.1 Kinematic method . . . . . . . . . . . . . . . . . . . . . . . 260

9.1.1 ASFIN: identification of undetected particles through momentum conservation . . . . 260

9.1.2 CHIRONE: background rejection through kinematic coincidences . . . . . . . . . . 261

9.1.3 NUCLEX: correlation analysis for rare event selection . . . . . . . . . . . . . . . . 262

9.2 Bayesian methods . . . . . . . . . . . . . . . . . . . . . . 263

9.2.1 ALICE: combining PID techniques using a Bayesian approach . . . . . . . . . . . 263

10 Summary . . . . . . . . . . . . . . . . . . . . . . . . 265

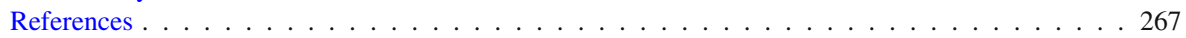

\section{Introduction}

To achieve their physics goals, nuclear physics experiments often need to identify particles or nuclei produced in the studied reactions. To this purpose, several techniques and detectors have been developed and improved over time. In recent years, the classical techniques based on $\Delta E-E$, pulse shape analysis, gamma detection, time of flight, calorimetry and Cherenkov light have been adapted to the new possibilities offered by the use of solid-state sensors, new developments in the detector technologies and new scintillator materials. New readout solutions offer the possibility to extract more information from the signals and at a faster rate. Sophisticated software approaches, based on a combination of different techniques, are demonstrating their validity in several analyses.

The third scientific Committee of the Istituto Nazionale di Fisica Nucleare (INFN), CSN3, deals with experiments covering different aspects of nuclear physics reactions [1]. Twenty five experiments are followed by the Committee and are located in several national and international laboratories. The nuclear reactions studied cover a huge interval of center of mass energies, from sub-eV up to $\mathrm{TeV}$ at high energy colliders. As a consequence, each experiment developed specific and challenging solutions for its particle identification needs, adapting the techniques to the interval of energies to be covered, from $\mathrm{eV}$ particles produced in low energy machines up to $\mathrm{GeV}$ ones produced at colliders. These needs allowed the INFN groups to gain a huge expertise in the development and operation of detectors for Particle IDentification (PID).

The present paper will review the acquired knowledge, explaining the different technical solutions and the achieved performances and results. The main emphasis will be on the techniques used, the specific performances depending on the needs of 
the experiments and being not directly comparable. As it will become clear in the present review, PID is not only a way to identify particles or nuclear states and study their properties, but also offers a strategy to distinguish the type of reaction on the basis of the final products. The review will focus on active projects and will concentrate on the contributions of the Italian groups. A description of the state of the art is particularly timely in view of the new experimental scenarios connected to the upgrade programs of the accelerator facilities in the INFN National Laboratories of Legnaro (LNL) [2], Catania (LNS) [3-5], L'Aquila (LNGS) [6] and the new physics possibilities offered by CERN-LHC and BNL-EIC colliders [7].

The review is organized as follows: after a brief introduction of the different experiments, the various PID analyses are grouped according to the used technique and they are described in detail by means of specific applications.

\section{Nuclear physics experiments in CSN3 implementing PID techniques}

In this section, the experiments contributing to the present review are briefly described with their main physics goals and the developed PID techniques. The experiments in CSN3 are organized into six different research lines that match the NUPECC ones (Nuclear Physics European Collaboration Committee [8]): (i) Quark and hadron dynamics studying the hadron internal structure and the strong force among quarks, (ii) Phase transition of nuclear and hadronic matter concentrating on high energy ion collisions and Quark-Gluon Plasma (QGP) studies, (iii) Nuclear structure and reaction dynamics with a variety of measurements along the chart of nuclides to understand the characteristics of each element and the fundamental laws that govern the chart, (iv) Nuclear astrophysics pointing at low-energy reactions of interest for stellar evolution and nuclear species abundance studies, (v) Symmetries and fundamental interactions studying basic physics quantities like the nucleon radius or matter/antimatter symmetry, (vi) Applications and societal benefits performing measurements useful in hadrotherapy or protection in space or for time metrology.

Although every experiment has its own specific goals and studies various reactions at different energies, there are many cross-connections among them. A clear example of this synergy among the different experiments in CSN3 can be found in the field of Nuclear Astrophysics [9].

In the following, each experiment is identified by the name of the corresponding international collaboration or by the acronym used within the INFN CSN3. The experiments are mostly performed inside INFN National Laboratories in Frascati (LNF), Legnaro (LNL), Catania (LNS) and Gran Sasso (LNGS), but also in several international laboratories like CERN, Jefferson Lab, GANIL, GSI, RIKEN.

\subsection{Quark and hadron dynamics}

\subsubsection{EIC: electron ion collider at Brookhaven National Laboratory (BNL)}

In spite of undoubted successes of QCD and its continuous progress, important aspects are still eluding our understanding. Among them, the emergence of the global nucleon 
properties, like mass and spin, from the dynamics of quarks and gluons inside the finite size nucleons; the full 3-D description of the nucleons; the properties of dense gluonic systems [10-14]. A complete picture can emerge only from a wide and deep systematic exploration, where the use of lepton probes has been demonstrated as the most successful approach. This is the mission of the electron ion collider (EIC), currently an approved project in USA $[7,15,16]$.

The EIC will be hosted at the BNL and will be the first electron-ion collider capable of accelerating a wide variety of ions, from hydrogen to uranium, and providing highly polarized $(\sim 80 \%)$ electron and light nuclei beams. The center-of-mass energy will span a wide range from 20 to $141 \mathrm{GeV}$ with peak luminosity above $10^{34} \mathrm{~cm}^{-2} \mathrm{~s}^{-1}$. The collider opportunities have to be exploited by detectors with full $4 \pi$ coverage, to ensure a complete exploration of the interaction products. The first collider operation is scheduled in 2031 .

Several key elements of the EIC physics program require excellent identification of the produced hadrons [17-21], and demand for improved and novel PID techniques. INFN groups are preparing their participation in the EIC experimental program largely contributing to the R\&D studies in this sector. A particular development is being pursued on Ring Imaging CHerenkov (RICH) detectors, with different technical solutions allowing particle separation in the momentum range from few hundred $\mathrm{MeV}$ up to 60 $\mathrm{GeV} / \mathrm{c}$ (see Sect. 8.2).

\subsubsection{JLAB: studies on hadron structure at Jefferson Lab}

The JLAB Collaboration groups researchers working at Jefferson Lab (USA) at the Continuous Electron Beam Accelerator Facility (CEBAF), recently upgraded to $12 \mathrm{GeV}$ energy. The main physics goal is a deeper understanding of the structure of the hadrons, their parton content and the different contributions to their spin, also by means of polarized beams and targets. The collaboration contributes to several experiments in the two main halls of the facility.

In particular, the CLAS12 detector [22] has been designed to carry an extensive physics research program [23], with two main aims. First, an innovative study of the $3 \mathrm{D}$ nucleon structure and of the underlying parton dynamics is planned in the valence quark region. This requires an excellent hadron identification to access flavor separation, and such a goal is achieved with an Aerogel RICH counter, able to identify pions, kaons and protons in the energy range 3-8 GeV [24] (see Sect. 8.3). Second, search for exotic states of the matter, like rare $q \bar{q}$ states and unconventional mesons with exotic quantum numbers [25]. This is pursued in CLAS12 by tagging events of quasi photo-production with a small-angle lead-tungstate calorimeter, complemented by tracking and pre-shower systems and covering an electron energy interval $0.5-$ 4.5 GeV (see Sect. 7.2).

\subsubsection{KAONNIS: studies of Kaonic atoms at DAФNE LNF}

The KAONNIS collaboration includes a series of projects focusing on the Kaonic atoms that represent a unique opportunity to study low-energy (almost zero) QCD in the strangeness sector [26,27]. The nature of the strong interaction between strange 
mesons and nucleons at threshold (almost zero relative kinetic energy), where a perturbative approach is not anymore valid, is indeed an important brick of the Standard Model to be still fully understood. A kaonic atom is formed when a low momentum $\mathrm{K}^{-}$is stopped in a target and is captured in an atomic orbit, replacing an electron: due to its much heavier mass, the kaonic atom is formed in a highly excited state. The subsequent radiative transitions of the $\mathrm{K}^{-}$towards the fundamental level, where QCD effects overlap with the QED ones, represent a signature of the kaonic atom formation and can be used to retrieve information on the kaon-nucleon $(\mathrm{KN})$ interaction. Typical energies of these transitions lay in the X-ray range: high-resolution spectroscopic detectors, like silicon drift detectors (SDDs) [28,29], represent the ideal tool for their precision measurements.

Within the collaboration, in 2009, the SIDDHARTA experiment at the DA $\Phi$ NE collider at LNF [30,31] performed a data taking campaign resulting in the highest precision measurement of $\mathrm{KH}$, the first measurement of $\mathrm{K}^{4} \mathrm{He}$ in a gaseous target and the first measurement ever of $\mathrm{K}^{3} \mathrm{He}$ [32-35].

To achieve these results, the combined spectroscopic and timing capabilities of a scintillator pair (see Sect. 6.1.2) and a set of $1 \mathrm{~cm}^{2}$ SDDs have been used (see Sect. 5.2). Background reduction is mandatory to perform a successful measurement: this is achieved combining SDDs and time of flight information to trigger the DAQ.

In 2021-2022, the SIDDHARTA-2 experiment should perform the first measurement ever of kaonic deuterium [36].

\subsubsection{MAMBO: meson photoproduction on nucleon in the BGOOD experiment}

The INFN-MAMBO Collaboration studies hadron photoproduction with and without polarization at different accelerators: ELSA (ELectron Stretcher Accelerator), delivering (un)polarized electrons in the $0.5-3.5 \mathrm{GeV}$ energy range, in Bonn and MAMI (the Mainz Microtron). The BGOOD experiment, working at ELSA, is designed to investigate the hadron structure, studying exclusive channels of meson photoproduction on the nucleon [37-39]. It consists of two main parts: (1) a central detector with a Bismuth Germanium Oxide (BGO) crystal calorimeter, a plastic scintillator barrel and two Multi Wire Proportional Chambers (MWPCs); (2) a forward large aperture magnetic spectrometer. The photon beam (with energies in the interval 0.5-3.2 GeV), linearly polarized and tagged in energy, impinges on a liquid $\mathrm{H}_{2} / \mathrm{D}_{2}$ target. The detector is designed to measure charged particles, neutrons and gammas. In Sect.7.3, the identification of neutrons and gammas in the BGO detector, necessary for the reconstruction of neutral meson decay, is described.

\subsection{Phase transition of nuclear and hadronic matter}

\subsubsection{ALICE: QCD studies in extreme conditions with heavy-ion collisions at the CERN LHC}

The ALICE experiment $[40,41]$ is dedicated to the study of $\mathrm{pp}, \mathrm{p}-\mathrm{Pb}$ and $\mathrm{Pb}-\mathrm{Pb}$ collisions provided by CERN LHC and investigates the properties of the strongly 
interacting matter at extremely high temperatures and densities. In such conditions, the nuclear matter undergoes a phase transition, creating a new state of matter where quarks and gluons are not confined into hadrons, known as the quark-gluon plasma (QGP). Moreover the experiment, by studying the dependence of the particle production with respect to the multiplicity of tracks in the event, has demonstrated the presence of QGP-like effects also in small collision systems (pp) and a smooth connection of the production mechanisms in different interactions ( $\mathrm{pp}, \mathrm{p}-\mathrm{Pb}$ and $\mathrm{Pb}-\mathrm{Pb}$ ), opening important windows towards a unified QCD description of these different size colliding systems. In addition, a series of studies on light (anti)nuclei production and annihilation gave precise measurements that allow a better understanding of nuclei formation and constrain the search for dark matter. Results from the RUN1 data taking period are summarized in [42], while more recent results can be found in [43].

To accomplish the physics goals, it is fundamental to have identification of particles produced in a wide momentum range. ALICE has unique particle identification capabilities among the LHC experiments; it exploits different PID techniques in the central barrel region, i.e., energy loss (see Sect. 3.3), time of flight measurements (see Sect. 6.1.1), Cherenkov and transition radiation detection (see Sect. 8.1), calorimetry (see Sect. 7.1.1) and topological ID (see Sect. 9.2.1). At forward rapidity, ALICE is equipped with a dedicated muon spectrometer to accomplish quarkonia states study (see Sect. 7.1.2).

\subsection{Nuclear structure and reaction dynamics}

\subsubsection{CHIRONE: heavy-ion nuclear reactions in a wide range of energies}

The CHIRONE experiment operates in the field of nuclear reactions, covering a wide energy range, from few MeV/nucleon to the Fermi energy (around $40 \mathrm{MeV} /$ nucleon), and using stable and unstable ion beams. In this framework, many physics cases have been studied, such as: isospin effects on formation and decay mechanisms of compound nuclei, dynamical and thermodynamical effects on nuclear reactions, structure of weakly bound light nuclei, collective behaviour of nuclei, symmetry energy term of equation of state (EoS) and clustering of light nuclei with also nuclear astrophysics implications [44-48].

The complete nuclei identification from light to heavy ones and the determination of their direction of emission in momentum space are the basis to construct observables needed for the analyses. The CHIRONE experimental group operates mainly using the multidetector CHIMERA [44] and the FARCOS array [49] at LNS, where various identification techniques are applied. The $\Delta E-E$ method is used to get the charge and mass of ions punching through the first stage of CHIMERA and FARCOS telescopes (see Sect. 3.2.1), the pulse shape analysis (PSA) in silicon detectors is used to obtain the charge of ions stopped in the first stage of CHIMERA telescopes (see Sect. 4.2.1), the PSA in scintillators is used to get charge and mass of Light Charged Particles $(Z<5)$ stopped in the $\mathrm{CsI}(\mathrm{Tl})$ and for the $\gamma$ identification (see Sects. 4.1.1, 4.3.1 and 5.1). Moreover, time of flight is used for measurements of velocity and mass determination of ions stopped in the first stage of telescopes (see Sect. 6.2.2) and, in 
the case of reactions with few emitted particles, the use of kinematic methods allows to extrapolate energy and angular distributions with an improved accuracy, allowing a better identification (see Sect. 9.1.2). Combining these methods, the collaboration gets an almost complete event characterization, i.e., charge/mass, energy and velocity of all the charged particles and $\gamma$-rays.

\subsubsection{FORTE: dynamics of fission and shell effects in super-heavy elements}

The FORTE collaboration aims at studying the role of shell effects in reactions between heavy ions finalized to the search of Super Heavy Elements, and the production of nuclei very rich in neutrons populating the so called Terra Incognita. Shell effects embrace the entire chart of nuclides. To study their importance on the heavy and superheavy mass region, the following processes are the main focus of the collaboration: (1) fission, quasi-fission and ternary fission of intermediate mass, heavy and superheavy nuclei; (2) production mechanisms (multinucleon transfer and surrogate reactions) and properties of exotic neutron-rich species in the unknown nuclear chart regions, including those of astrophysical interest. The collaboration operates in several laboratories like LNL, ISOLDE and GSI.

The research program is implemented mainly using a time of flight spectrometer (TOF) coupled, whenever required by the experimental aim, with charged particles, neutrons and gamma-ray detectors. The TOF spectrometer measures masses and total kinetic energies of fragments in binary reactions (see Sect. 6.2.4). As an example, these quantities, measured in coincidence with the light charged particles detected by the 250 bi-telescopes of $8 \pi \mathrm{LP}$ at LNL in the fusion-evaporation and other channels [5053], allow to estimate the reaction cross sections, to obtain a better understanding of the dynamics of processes occurring in heavy-ion induced reactions. This TOF spectrometer is well suited for both stable beams, presently available at LNL and future radioactive ion beams [54] produced by SPES [55]. Typical studies explore fission-dynamics [56,57] or the separation of the binary products of fast reactions (e.g. deep-inelastic and quasi-fission) from those of fission [58,59], to get insight on the scission point configuration, e.g., deformation, mass asymmetry and dissipated energy. The compact size, light weight and easy-to-handle design of the TOF spectrometer makes it a portable solution and has recently been an important component of the experimental campaign performed at JYFL laboratory (Finland) focusing on the study of the ternary fission in heavy-ion-induced reactions and the fission modes in mercury isotopes.

\subsubsection{GAMMA: study of the nuclear structure using $\gamma$ spectroscopy}

The GAMMA collaboration aims at performing high precision $\gamma$ spectroscopy to make several studies covering, for example, the nuclear structure in regions far from stability, critical points and phase transitions between different nuclear shapes, collective modes of nuclei (vibration and rotation), resonances and particle/phonon coupled states, also of interest for astrophysics. To achieve this rich program the collaboration is involved in several experiments at different laboratories (like LNL, GANIL, GSI, IJC...), with 
specific devices for $\gamma$ and particle measurements, such as high-purity Germanium detectors (HPGe), silicon detectors and organic/inorganic scintillators.

Silicon detectors are widely used to perform X-ray and particle spectrometry. Due to the natural higher defect and contaminants density in silicon with respect to germanium, their thickness is limited to few $\mathrm{mm}$. In such detectors, the position sensitivity is generally given by the electrode segmentation and the eventual evaluation of the charge sharing between neighbouring segments. In GAMMA the application of PSA to signals coming from silicon detectors is used to discriminate the impinging particles in charge and mass (see Sect. 4.2.2).

With the same approach, PSA is applied to organic and inorganic scintillators to discriminate incoming radiation resulting from mixed-field sources. In particular, it is possible to combine different scintillating materials with different signal shapes to obtain advantages in terms of efficiency, resolution and availability (see Sect. 4.3.2 ).

HPGe arrays are characterized by high-energy resolution that is however degraded when measuring in-flight emitted $\gamma$-rays, because of the indetermination in the measurement of the associated recoiling nucleus momentum. Many solutions adopted to overcome this limitation have undesired effects, like reduced acceptance or need for complementary detectors. An optimal strategy has been found in the PSA and $\gamma$-ray tracking techniques (see Sect. 4.4.1), adopted in their most advanced implementation by the AGATA detector array [60].

The upcoming facilities for radioactive beams, like SPES [55] at LNL, will open up new and challenging research lines for the GAMMA collaboration [61] probing clusterization, shape coexistence, high-order deformation and other interesting nuclear structure phenomena far from the valley of stability.

\subsubsection{NUCLEX: nuclear dynamics and thermodynamics with heavy ions}

The NUCLEX collaboration studies the behaviour of nuclear matter in a wide range of beam energies (5-100 MeV/nucleon) using different setups and particle identification techniques. This diversification is needed to better investigate phenomena typical of different energy regimes: compound nucleus formation and decay [62], $\alpha$-clustering [63], charged particle spectroscopy [64], isospin transport mechanisms [65-67] and neck emissions [68]. Various setups have been developed and used at LNS, LNL and GANIL: FAZIA [69,70], GARFIELD [71], OSCAR [72] and the ACTAR demonstrator [73]. PID makes use of detectors based on combinations of sensors into telescopes (see Sect. 3). A particle, depending on its energy, may pass through one or more layers of the telescope: if it stops in the first layer it is possible to use PSA, Bragg spectroscopy (see Sects. 4.1.2, 4.2.3, 4.3.5) or time of flight techniques (see Sect. 6.2.5) to identify it, else the $\Delta E-E$ correlation is adopted (see Sect. 3.2.2). Kinematic methods are also extensively used (see Sect. 9.1.3) to identify nuclei at relevant excitation energies.

\subsubsection{NUMEN: nuclear matrix elements of neutrinoless double beta decays}

The NUMEN project [74] proposes an innovative technique to access the nuclear matrix elements entering the expression of the lifetime of the double beta decay. It 
is based on accurate cross section measurements of heavy-ion induced double charge exchange (DCE) reactions at energies of 15-60 MeV/nucleon [75-78]. The NUMEN experiments are performed at LNS using the K800 Superconducting Cyclotron (SC) and MAGNEX large acceptance magnetic spectrometer to detect the emitted ions [79, 80]. MAGNEX is a powerful and versatile device that has been successfully used in a variety of nuclear physics researches [81-86]. The explored DCE reactions are induced by ${ }^{18} \mathrm{O}$ and ${ }^{20} \mathrm{Ne}$ beams, which imply the identification of ${ }^{18} \mathrm{Ne}$ and ${ }^{20} \mathrm{O}$ ejectiles, respectively. In addition, NUMEN proposes to measure also the complete set of competing reactions, i.e. single charge exchange, multi-nucleon transfers [87], elastic and inelastic scattering [88]. The corresponding ejectiles are typically in the mass region $18 \leq A \leq 22$ and atomic number $8 \leq Z \leq 10$. Different charge states are distributed at the focal plane for each isotope species, due to the interaction with the target [89], making the ion identification more challenging. The MAGNEX PID technique is based on a combination of measurements of energy loss, residual energy and magnetic rigidity (see Sect. 3.2.3) and guarantees a clear selection of the ions of interest among the whole range of $A$ and $Z$ produced in the collision.

\subsubsection{PRISMA: heavy-ions dynamics with a large angle magnetic spectrometer at LNL}

Binary reactions involving heavy ions allow to study the dynamics and the structure of nuclei in a wide range of angular momenta and excitation energies. In particular, with multi-nucleon transfer reactions it is possible to study the residual interaction in nuclei, especially the components responsible for nucleon coupling and correlations. Magnetic spectrometers equipped with proper detectors are well suited to analyze and interpret such reactions because they allow measuring at the same time mass and charge of reaction products, differential and total cross sections, and total kinetic energy.

In the experiment PRISMA at LNL [90], the development of a magnetic spectrometers covering large solid angles, coupled with precise ion tracking and an efficient time of flight measurement (see Sect. 6.2.6), allowed the improvement of the detection efficiency and mass resolution for very heavy ions, with important results in the field of nuclear structure and nuclear reaction dynamics. As an example, measurements of transfer probabilities in multi-nucleon transfer reactions at energies around the Coulomb barrier [91] or far below the Coulomb barrier [92] shed light on the nucleon pairing interaction [93]. When coupled to large $\gamma$ detector array like CLARA, the PRISMA spectrometer has allowed studies of particle-particle and particle-core couplings, thanks to the ability to detect individual transitions and study their population pattern and decay modes [94,95]. For example, the analysis of particle- $\gamma$ coincidences resulted in a substantial suppression of $\gamma$-ray background and therefore provided improved sensitivity [96,97].

The forthcoming new collaboration with the AGATA (see Sect. 2.3.3) array and the availability of radioactive ion beams provided by SPES will allow to unveil the structure and reaction dynamics of exotic nuclei even further away from the stability valley [2]. 


\subsection{Nuclear astrophysics}

\subsubsection{ASFIN: nuclear astrophysics studies at very low energy}

Stellar evolution is strictly related to the energy production due to nuclear reactions, which are also responsible for the synthesis of the elements. Owing to the presence of the Coulomb barrier at kinetic energies relevant for astrophysics, it is very difficult, or sometimes impossible, to measure astrophysical reaction rates in the laboratory. Consequently, trying to avoid extrapolations, alternative methods for determining bare nucleus cross sections of astrophysical interest are necessary [98], such as the TrojanHorse Method (THM).

The method has been applied to nuclear structure and interaction problems, but mostly to hotly debated nuclear astrophysics issues (see [99] for the latest review). Recently, the method has been extended to the indirect study of neutron-induced reactions [100] and to reactions of astrophysical interest induced by radioactive ion beams [101], opening a new field of research overcoming the experimental difficulties related to the direct measurements of neutron-induced reactions with unstable beams [102].

The peculiarity of the method and the typical low energy range of a nuclear astrophysics experiment requires a non-trivial application of the standard particle identification techniques. Indeed, the needs of low-energy threshold detection triggered the realization of monolithic $\Delta E-E$ detectors (see Sect. 3.1.2), while the necessity to measure reactions with three bodies in the final state inspired a kinematic method to determine the mass number of an undetected particle (see Sect. 9.1.1). To identify a specific reaction mechanism, a time of flight measurement can be applied (see Sect. 6.2.1). All these techniques were largely applied in the nuclear astrophysical research of the ASFIN group at the principal Italian national laboratories (LNS and LNL) and international ones (TRIUMF, RIKEN, GANIL...) using advanced experimental setup (CLAD [103], TECSA [104] and many others).

\subsubsection{ERNA: direct measurements for $\mathrm{C}$ burning in stars}

The main goals of the ERNA collaboration are cross section measurements of astrophysical interest with a mass recoil separator, performing charged particles spectroscopy, and the determination in meteoritic samples of the isotopic mass composition through mass spectrometry. For charged particle spectroscopy the collaboration designed a large scattering chamber where either a solid or a gas jet targets can be located, in combination with different detection setups.

A special case to illustrate the experimental challenges is the ${ }^{12} \mathrm{C}+{ }^{12} \mathrm{C}$ reaction, playing a key role in the evolution of massive stars and in explosive scenarios such as type-Ia supernovae and super-bursts in binary stars. Carbon burning takes place at typical temperatures of $5 \times 10^{8} \mathrm{~K}[105,106]$, corresponding to an energy range of interest, the Gamow window [107], equal to $E_{\mathrm{c} . \mathrm{m} .}=1.5 \pm 0.3 \mathrm{MeV}$ [108]. Cross sections are very small $\left(\ll 10^{-9} \mathrm{~b}\right)$ and extremely difficult to measure in the laboratory due to critically low counting rates $[109,110]$, making it necessary to increase as much as possible the signal-to-noise ratio. At these energies, the ${ }^{12} \mathrm{C}+{ }^{12} \mathrm{C}$ reactions proceed 
mainly through the ${ }^{23} \mathrm{Na}+\mathrm{p},{ }^{20} \mathrm{Ne}+\alpha$, so that $\alpha$ and $\mathrm{p}$ channels identification plays a crucial role for channel selection and background suppression.

The ERNA collaboration at CIRCE laboratory in Caserta aims to study these reactions that require: (a) great stability to allow for long measurement campaigns, (b) low intrinsic background and (c) heat resistance to withstand the intense beams necessary to optimise the counting rate. PID at such low energies (few MeV/nucleon) and in such experimental conditions requires challenging detectors and ERNA developed a dedicated $\Delta E-E$ gas-silicon detector array, described in Sect.3.1.1.

\subsubsection{LUNA: study of nuclear reactions for astrophysics at LNGS}

The LUNA collaboration operates inside the LNGS under the Gran Sasso massif between the cities of L'Aquila and Teramo in Italy. This unique facility takes advantage of the environmental background reduction (for instance, a neutron background reduction by three orders of magnitude has been observed [111]) and of the intense beam provided by the LUNA accelerator to measure low-energy nuclear fusion reactions responsible for the synthesis of the elements in the Universe (see [112] and references therein). The main goal is to lower the minimum energy ever achieved with a direct measurement targeting the Gamow windows [107], therefore, reaching typical astrophysical temperatures. As an example, LUNA has measured the neutron source for the main s-process ${ }^{1}$ : the ${ }^{13} \mathrm{C}(\alpha, n){ }^{16} \mathrm{O}$ reaction. In this case a yield of about 1 neutron per Coulomb beam charge is expected. For this reason, the background must be minimized to have a signal-to-background ratio as high as possible and to limit the overall uncertainty to $10 \%$ as requested by model calculations [113].

Besides the naturally low-noise environment, further solutions were used to minimize background, for which PID plays a pivotal role. The background reduction was achieved in three steps:

- the production and characterization of pure $99 \%$ enriched ${ }^{13} \mathrm{C}$ solid targets minimized beam induced background [114]

- the selection of materials and the usage of counters with stainless steel case (instead of typical aluminium case) reduced the intrinsic $\alpha$ background by one order of magnitude [115]

- an improved PSA analysis method allowed to distinguish signals from neutron capture in the ${ }^{3} \mathrm{He}$ gas from residual $\alpha$ signals of the intrinsic background [116] (see Sect. 4.3.3).

\subsection{4 n_TOF: neutron time of flight facility at CERN}

The neutron time of flight facility n_TOF at CERN is a white neutron source based on the CERN Proton Synchrotron (PS). A high-intensity neutron beam is produced every $1.2 \mathrm{~s}$ (or multiples) from a pulse of $7 \times 10^{12}$ protons of $20 \mathrm{GeV} / \mathrm{c}$ momentum accelerated by the PS, and impinging on a massive lead target, surrounded by $5 \mathrm{~cm}$ of water. At a distance of about 185 and $20 \mathrm{~m}$ from the spallation source the two experimental areas,

\footnotetext{
${ }_{1}$ Through this process about half of the elements heavier than iron are synthesized, through a sequence of neutron capture reactions and subsequent $\beta$ decays [107].
} 
EAR-1 and EAR-2 [117,118], are placed. The characteristic experimental conditions of n_TOF ensure a high instantaneous neutron flux, which covers a wide energy range, from thermal energy to a few $\mathrm{GeV}$. The neutron-induced reactions studied so far are radiative capture (n, $\gamma$ ), fission (n, f), charged particle emission (n, c.p.) and inelastic scattering $\left(n, n^{\prime}\right)$, of interest to fundamental nuclear physics, stellar nucleosynthesis or applications in nuclear technology and nuclear medicine [119]. Cross section studies require the simultaneous measurement of the products of the reaction and of the neutron flux impinging on the sample.

PID techniques are mainly applied to experimental data collected for (n, $\gamma$ ) cross section measurements, which typically involve neutrons with energy from thermal to few hundreds keV. The purpose of the applied PID techniques is twofold: to identify $\gamma$ rays from the radiative capture reaction and other background particles, i.e. neutrons or $\alpha$ particles, and to monitor the stability of detector gains (see Sect. 4.3.4). In the near future the collaboration is going to start a measurement campaign of (n, c.p.) reactions of interest to fusion technology, where PID represents a basic requirement. In addition, new counter telescopes developed by the collaboration make extensive use of the $\Delta E-E$ technique combined with time of flight method to measure the neutron flux in the high-energy region, from $10 \mathrm{MeV}$ to approximately $1 \mathrm{GeV}$ (see Sect. 6.1.3).

\subsubsection{PANDORA: plasma for astrophysics, nuclear decays observation and radiation for archaeometry}

The PANDORA project [120,121] proposes a new approach to measure, for the first time, in-plasma nuclear $\beta$-decays of isotopes of astrophysical interest (e.g. relevant for $\mathrm{r}$ - and s-process nucleosynthesis chain), covering different plasma densities $n_{e}$ and temperatures $T_{e}$ [122]. Theoretical predictions and former experiments on fully stripped ions have shown that the ionization state can modify, even of several orders of magnitudes, the isotopes lifetimes [123-126]. In the high-performance PANDORA plasma trap, the radionuclides can be trapped in a dynamic equilibrium, living for days or even weeks at almost constant local density and temperature. The plasma may reach $n_{e} \sim 10^{11} \div 10^{13} \mathrm{~cm}^{-3}, T_{e} \sim 0.1 \div 100 \mathrm{keV}$ and mimic some stellar-like environments concerning, mainly, the charge state distribution (CSD) conditions.

The experiment studies the $\beta$ decays of the radionuclides in the plasma trap via the identification and measurement of the $\gamma$ rays emitted from the excited states of the daughter nuclei and distinguishes the background coming from the plasma itself. This is obtained by means of a multi-diagnostic setup and HPGe detectors, as described in Sect. 5.3.

\subsection{Applications and societal benefits}

\subsubsection{FOOT: nuclear cross section measurements for hadron therapy}

The goal of the FOOT (FragmentatiOn Of Target) experiment [127] is to perform cross section measurements of nuclear fragmentation of projectiles and targets that are rel- 
evant for particle therapy and radiation protection in space. Specifically, FOOT will measure double differential (with respect to fragment kinetic energy and production angle) cross sections, using thin tissue-like $(\mathrm{C}, \mathrm{O}, \mathrm{H})$ targets and light $(\mathrm{Z} \leq 8)$ projectiles with energies in the range 100-800 MeV/nucleon. Data of this kind are scarcely available. New measurements will provide important reference data sets for model benchmark, allowing a better understanding of the physical and biological effects of secondary particles produced in the human body during tumor irradiation in particle therapy and during exposure to space radiation in long-term space missions.

While projectile fragmentation can be studied directly, for target fragmentation studies involving very short range and low-energy fragments, FOOT applies an 'inverse kinematics approach'. To have a clear separation of the isotopes needed for the cross section measurements, the identification capabilities of the fragments in terms of charge $Z$ and mass $A$ should reach a level of precision of about 3 and 5\%, respectively. Other constraints come from the fact that the experiment should be movable since the data taking periods are foreseen at different facilities. Based on the expected kinematic ranges of the fragments to be studied, two distinct experimental setups have been developed for FOOT:

- a setup optimized for fragments with $Z \geq 3$, including a magnetic spectrometer, coupled with a tracking region, time-of-flight and calorimeter, covering up to about $20^{\circ}$ (see Sect. 6.2.3).

- a setup optimized for fragments with $Z \leq 2$, consisting of an emulsion spectrometer placed behind the beam monitor, with acceptance of up to about $70^{\circ}$ (see Sect. 3.4).

\section{The $\Delta E-E$ technique}

In the case of non relativistic charged particles, a commonly adopted approach to their identification is the measurement of their energy loss in a material with a thickness insufficient to stop the particle. Indeed, when a charged particle traverses matter it undergoes a number of interactions, mainly inelastic collisions with the atomic electrons of the material and elastic scattering of atomic nuclei. Most frequently, collisions cause very small energy losses in comparison with the impinging energies (for $90 \%$ of all collisions the energy losses are less than $100 \mathrm{eV}$ [128]). The cumulative effect of the many interactions is a sizable energy loss of the impinging particle and a deflection with respect to the original trajectory. Though the nature of these interactions is definitely stochastic, if the distance across the material is macroscopic then the number of collisions is very high and fluctuations are small, so an average energy loss can be unequivocally determined. The problem was already tackled at the very beginning of nuclear physics (Bohr's formula dates back to 1913 [129]), and the energy loss per unit path (the so-called stopping power) is accurately described by the Bethe-Bloch formula (see [128] for a detailed discussion). The stopping power $\langle-\mathrm{d} E / \mathrm{d} x\rangle$ decreases with $\sim 1 / \beta^{2}$ until about $\beta \gamma \sim 3$, then a broad minimum is reached (minimum ionizing particles-MIPs in the following) and a weak increase is finally observed for relativistic energies. A general feature that makes stopping power very attractive for 
particle identification is the apparent dependence on $Z^{2}$, where $Z$ is the charge of the incident particle, while a minor dependence on the particle mass $M$ is present, which is easily predicted in the non-relativistic limit:

$$
\left\langle-\frac{\mathrm{d} E}{\mathrm{~d} x}\right\rangle \sim \frac{Z^{2} M}{E} .
$$

The linear dependence on the incident particle mass makes it possible to perform identification of different isotopes in the case of heavy ions, provided that high resolution in the measurement of the energy loss is attained. Since sufficient energy resolution to discriminate in mass is seldom reached, the measurement of the energy loss $\Delta E$ is often coupled to other techniques. In the following subsections, we will discuss how the experiments use the $\Delta E-E$ approach for particle identification. Such technique is based on the construction of 2D spectra where the energy loss in a thin detector ( $\Delta E$ stage) is drawn as a function of the residual energy measured in a thicker detector where the particle is stopped (the combination of the two-or more-detectors is often referred to as a telescope).

\subsection{The low-energy limit: research in nuclear astrophysics}

As discussed in 2.4, nuclear astrophysics experiments are characterized by very low (from $\mathrm{keV}$ to $\mathrm{MeV}$ ) beam energies and cross sections. PID is therefore a challenge in terms of energy thresholds in detectors and signal-to-noise ratios.

\subsubsection{ERNA: advanced ionization chamber as $\Delta E$ detectors}

The very low energies involved in the study of nuclear reactions of astrophysical interest require to push the standard detection technique of $\Delta E$ to its limits. On one hand, a detector as thin as possible is necessary to measure the energy loss without introducing a significant threshold; on the other hand, energy loss cannot be too low to maintain good particle separation.

As an example, at low beam energies (6-8 MeV/nucleon), measurements of the ${ }^{12} \mathrm{C}+{ }^{12} \mathrm{C}$ fusion reaction, proceeding through $\mathrm{p}$ and $\alpha$ channels, have proved that the $\Delta E-E$ technique can successfully identify the emitted protons [130]. However, even the most energetic $\alpha$ particles are not visible if a sufficiently thin $\Delta E$ stage is not used. Such phenomenon is especially true when the reaction channels involve excited states of ejectiles.

To overcome the threshold problem, within the ERNA collaboration a two-stage detector array called GASTLY (GAs Silicon Two-Layer sYstem) was specifically designed to fulfill the requirements of charge-particle measurements at low energies. The GASTLY array can accommodate up to eight $\Delta E-E$ modules, each including an ionisation chamber (IC, $\Delta E$ stage) and a silicon strip detector (SSD, $E_{\text {rest }}$ stage) [131], spanning an angular range of about $70^{\circ}$ with a resolution $<1.3^{\circ}$. Each module consists of an aluminium pyramidal structure with square base. The IC is operated with $\mathrm{CF}_{4}$ maintained at a constant pressure that can be adjusted from 0 to 250 mbar (limit 


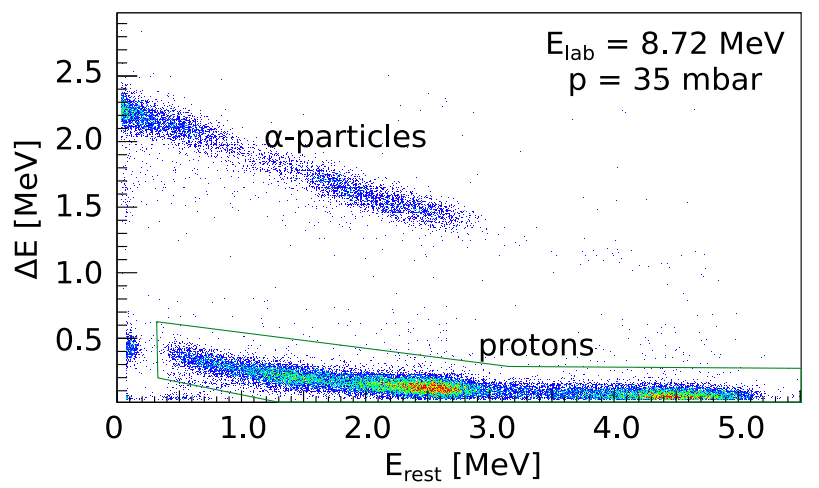

Fig. 1 ERNA. Typical energy calibrated $\Delta E-E_{\text {rest }}$ scatter plot showing the $\alpha$ particles and protons loci. The figure is taken from [133]

imposed by the entrance window) to fit specific needs of different energy ranges or reactions [131], obtaining in this way a $\Delta E$ stage as thin as needed. The second stage of each GASTLY module consists of a large $\left(58 \times 58 \mathrm{~mm}^{2}\right.$ active area, $300 \pm 15 \mu \mathrm{m}$ thick) SSD [132]. A typical $\Delta E-E$ matrix is shown in Fig. 1.

Using four modules of the GASTLY array and the SSD as a whole [133], it was possible to identify the ${ }^{2} \mathrm{H}$ contamination in the target allowing to perform a detailed study for beam-induced background reduction (see Ref. [134]). In addition, it was possible to test the optimum stability of the GASTLY system (2-week-long measurements), its great heat resistance (up to $40 \mu \mathrm{A}$ on target and $1400^{\circ} \mathrm{C}$ ) and extremely low intrinsic background $\left(10^{-2}\right.$ counts/h).

Currently, GASTLY is being tested at the LNGS to further investigate intrinsic background, with the aim of measuring the ${ }^{12} \mathrm{C}+{ }^{12} \mathrm{C}$ reactions directly in the Gamow window.

\subsubsection{ASFIN: pushing the low-thickness limit of solid-state devices}

The use of ionization chambers as $\Delta E$ stage of a conventional $\Delta E-E$ telescope ensures low detection thresholds and has been largely and successfully applied in THM experiments (see Sect. 2.4.1) and in the study of nuclear structure close to the particle emission thresholds [135]. Typical drawbacks are the impossibility to reach compact dimensions and the difficulty of setting up $4 \pi$ array for applications to radioactive ion beams. Si detectors allow one to build compact detection systems but such detectors are commercially available only down to about $5 \mu \mathrm{m}$ thickness, and such thin detectors are fragile and extremely expensive. Moreover, the high capacitance makes their performance unsuitable for nuclear astrophysics experiments.

To overcome these difficulties, some prototypes of monolithic silicon telescopes were tested at LNS, where the $\Delta E$ and $E$ stages are integrated in the same silicon chip, following a fundamental work of Kemmer and other groups [136]. Basically, a monolithic telescope is a $\Delta E-E$ detector with the structure of a double vertical diode built on a unique silicon bulk. A schematic view of the detector is reported in Fig. 2a. 

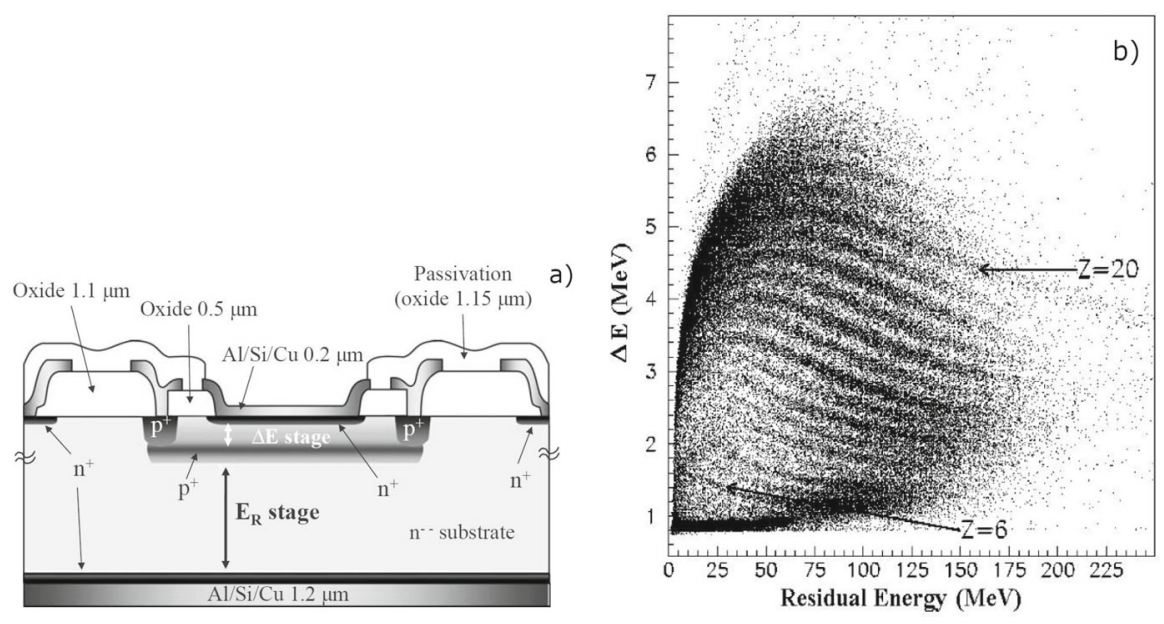

Fig. 2 ASFIN. a Sketch (not to scale) of the monolithic telescope structure. Particles enter the detector from the top. b $\Delta E-E$ spectrum for ${ }^{40} \mathrm{Ca}+{ }^{48} \mathrm{Ca}$ collision at $E=400 \mathrm{MeV}$. Both pictures are from [137]

The $\mathrm{p}+$ region acts as common ground electrode for the $\Delta E$ and residual energy stages. Details on the sensor manufacturing can be found in [138]. Several devices were realized, manufactured by STMicroelectronics (Catania, Italy) in the framework of a collaboration with LNS, by combining photolithographic and ion implantation techniques. In this way, it was possible to obtain telescopes with a very thin (down to $2 \mu \mathrm{m}$ ) first stage, leading to a $\Delta Z / Z \sim 10 \%$.

Monolithic strip detectors were extensively used in several experiments at low and intermediate energies, both with stable and radioactive beams [139], showing their powerful features: modular structure with possibility to build very compact and versatile configurations; charge identification thresholds of the order of hundreds $\mathrm{keV} /$ nucleon for ions up to $Z=20$; good granularity; excellent $\beta$ background suppression in reactions induced by radioactive beams and possibility to enhance the position sensitivity [140]. Figure $2 \mathrm{~b}$ shows a $\Delta E-E$ spectrum obtained in the study of the collision ${ }^{40} \mathrm{Ca}+{ }^{48} \mathrm{Ca}$ at a beam energy of $10 \mathrm{MeV} /$ nucleon.

Recently, a cooperation between LNS and IMM-CNR (Institute for Microelectronic and Microsystems of the Italian National Research Council) has lead to the development of a new generation of detectors made of Silicon Carbide (SiC) [141]. Since $\mathrm{SiC}$ devices present a wider bandgap than traditional silicon detectors, they are very robust against radiation damage and also present a better signal-to-noise ratio at high temperature. In view of those potential applications, the SiCILIA (Silicon Carbide Detectors for Intense Luminosity Investigations and Applications) project established a new era for $\mathrm{SiC}$ devices regarding thicknesses, dimensions (detection area), and manufacturing technologies [141].

Among these advanced attributes, a brand new invention patent was recently deposited showing the possibility of building a monolithic multilayer SiC device [142]. This new telescopic detector will open new perspectives for studying reaction cross 

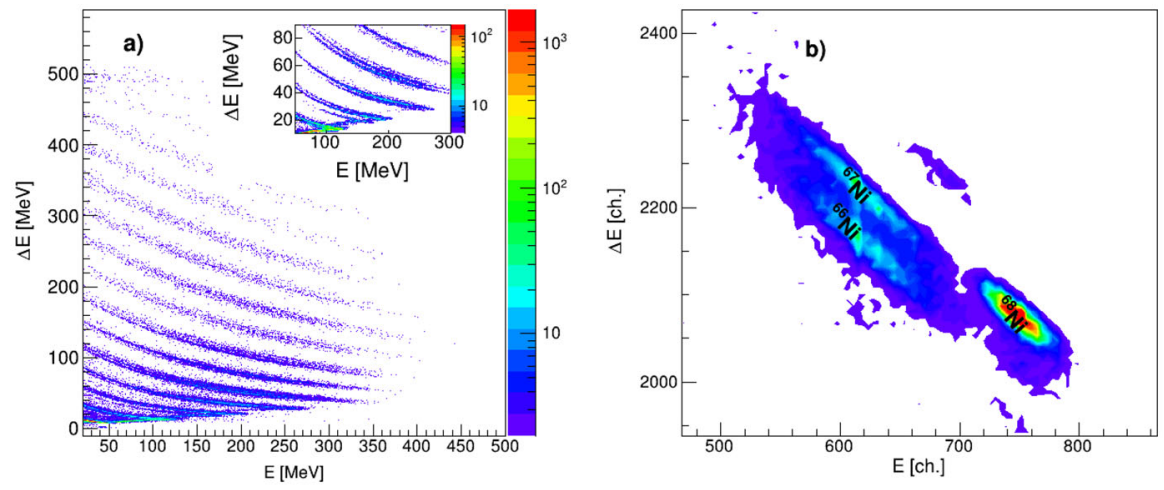

Fig. 3 CHIRONE. a $\Delta E-E$ plot obtained in the reactions ${ }^{124} \mathrm{Xe}+{ }^{64} \mathrm{Ni},{ }^{64} \mathrm{Zn}$ at $20 \mathrm{MeV} /$ nucleon using a FARCOS telescope. The inset shows a zoom of the $\Delta E-E$ plot for small $\Delta E$ values. b Uncalibrated $\Delta E-E$ plot obtained in the reaction ${ }^{68} \mathrm{Ni}+{ }^{12} \mathrm{C}$ at $28 \mathrm{MeV} /$ nucleon considering two subsequently crossed strips of a FARCOS telescope (adapted from [144])

sections under plasma environment of fundamental importance for nuclear astrophysics [143].

\subsection{Nuclear structure and dynamics experiments at intermediate energies}

In heavy-ion collisions in the range of the Fermi energy, new challenges arise for PID due to the large number of emitted particles, making it necessary to develop detectors with high granularity and good resolution in charge and mass.

\subsubsection{CHIRONE: energy loss in large acceptance arrays}

The $\Delta E-E$ method is largely applied, in both CHIMERA multidetector and FARCOS array (see Sect. 2.3.1). CHIMERA consists of 1192 telescopes and covers about the $94 \%$ of $4 \pi$. Each telescope consists of a $300 \mu \mathrm{m}$ Si detector followed by a CsI(Tl) from 3 to $12 \mathrm{~cm}$ thick [44]. The forward part of CHIMERA consists of 688 telescopes, distributed on 18 rings from $1^{\circ}$ to $30^{\circ}$. The backward part consists of 504 telescopes, distributed on a sphere from $30^{\circ}$ to $176^{\circ}$, with the target placed in the center. FARCOS is a modular array of telescopes, each of them consisting of two Double-Sided Silicon Strip Detectors (DSSSD), 300 and $1500 \mu \mathrm{m}$ thick, respectively, followed by a last stage including four CsI(Tl), $6 \mathrm{~cm}$ thick [49]. The final configuration of FARCOS consists of 20 telescopes.

The $\Delta E-E$ method in the CHIMERA multidetector uses the signals coming from Silicon detectors and $\mathrm{CsI}(\mathrm{Tl})$ scintillators to identify in charge all the fragments passing through the Si stage, and in mass the ones with $Z$ up to 10 (since $\Delta Z / Z \sim 2 \%$ and $\triangle \mathrm{A} / \mathrm{A} \sim 5 \%$ ). The same method is used to obtain particle identification with the FARCOS array; in this case signals coming from both the two silicon stages and second silicon stage-CsI(Tl) scintillator of a FARCOS telescope are used. Figure 3a shows a $\triangle E-E$ plot obtained using a telescope of the FARCOS array in the reactions 
${ }^{124} \mathrm{Xe}+{ }^{64} \mathrm{Zn},{ }^{64} \mathrm{Ni}$ at $20 \mathrm{MeV} /$ nucleon. Here the energy scale was optimized to detect Intermediate Mass Fragments. Figure $3 b$ shows a $\Delta E-E$ plot obtained in the reaction ${ }^{68} \mathrm{Ni}+{ }^{12} \mathrm{C}$ at $28 \mathrm{MeV} /$ nucleon. The $\Delta E-E$ plot was obtained considering a strip of the $300 \mu \mathrm{m}$ stage and the corresponding strip of the $1500 \mu \mathrm{m}$ detector, for a FARCOS telescope [144]. This figure shows the excellent performance of the FARCOS array allowing to identify in mass also nickel isotopes.

As it will be discussed in later sections, for the telescopes used in CHIRONE the $\Delta E-E$ technique, suitable for particles punching through the first detection layer, is complemented by other methods. In particular, the Pulse Shape Analysis in silicon, described in Sect.4.2.1, and in CsI(Tl), see 4.1.1, is used for particles stopped in the first layer and for light and fast particles, respectively. For particles stopped in the first layer also the E-TOF identification approach is adopted, as described in Sect. 6.2.2. Finally, the kinematic methods (see Sect. 9.1.2) are also used to improve the quality of the identification.

\subsubsection{NUCLEX: tailored $\Delta E-E$ solutions}

Different compositions of sensors within a telescope lead to different identification capabilities. Therefore, the $\Delta E-E$ combination has to be tailored to the physical problem under investigation. The NUCLEX collaboration has developed solutions incorporating solid-state devices only, such as FAZIA and OSCAR, and combination of gas and solid-state devices, as GARFIELD and ACTAR (see Sect. 2.3.4).

Regarding the former, FAZIA is made up of two silicon pads (300 and $500 \mu \mathrm{m}$ thick) followed by $10 \mathrm{~cm} \mathrm{CsI(Tl)} \mathrm{scintillators} \mathrm{and} \mathrm{it} \mathrm{is} \mathrm{designed} \mathrm{for} \mathrm{beam} \mathrm{energies}$ above $20 \mathrm{MeV} /$ nucleon, while OSCAR is devoted to the detection of low-energy (few $\mathrm{MeV} /$ nucleon) light charged particles, with a $20 \mu \mathrm{m}$ silicon strip detector followed by a $300 \mu \mathrm{m}$ Si pad wall.

GARFIELD is made up of two drift chambers surrounded by $1804-\mathrm{cm}$-thick CsI(Tl) scintillators; it is frequently coupled (towards forward polar angles) with an ancillary Ring Counter (RCo), which features ionization chambers followed by $300-\mu \mathrm{m}$-thick silicon strip pads and CsI(Tl) scintillators (third layer). The ACTAR demonstrator is a $64 \times 128 \times 170 \mathrm{~mm}^{3}$ active target and time projection chamber followed by ancillary detectors as a second layer (e.g. it has been recently coupled with OSCAR). GARFIELD is more suited for higher energies, in the 5-25 MeV/nucleon range while the ACTAR demonstrator is meant for beam energies up to $\sim 10 \mathrm{MeV} /$ nucleon (and detected particles of similar energies).

The advantage of the initial gas volume as in GARFIELD + RCo, transparent for fragments with at least $2 \mathrm{MeV} /$ nucleon, is the very low charge identification threshold, making it possible to measure at relatively low beam energies. However, the energy resolution of gas chambers does not permit the isotopic discrimination of fragments. For particles of energy high enough to reach the CsI(Tl) third layer in RCo, the $\Delta E-E$ technique could be exploited also correlating the energy lost in the Si layer with the residual energy loss inside the $\mathrm{CsI}(\mathrm{Tl})$ scintillators: in this case the determination of the mass number $\mathrm{A}$ is possible for particles up to $Z \sim 15$, due to the better energy resolution of the $\Delta E$ sensor $(\Delta \mathrm{A} / \mathrm{A} \sim 2.5 \%, \Delta Z / Z<2 \%)$. 


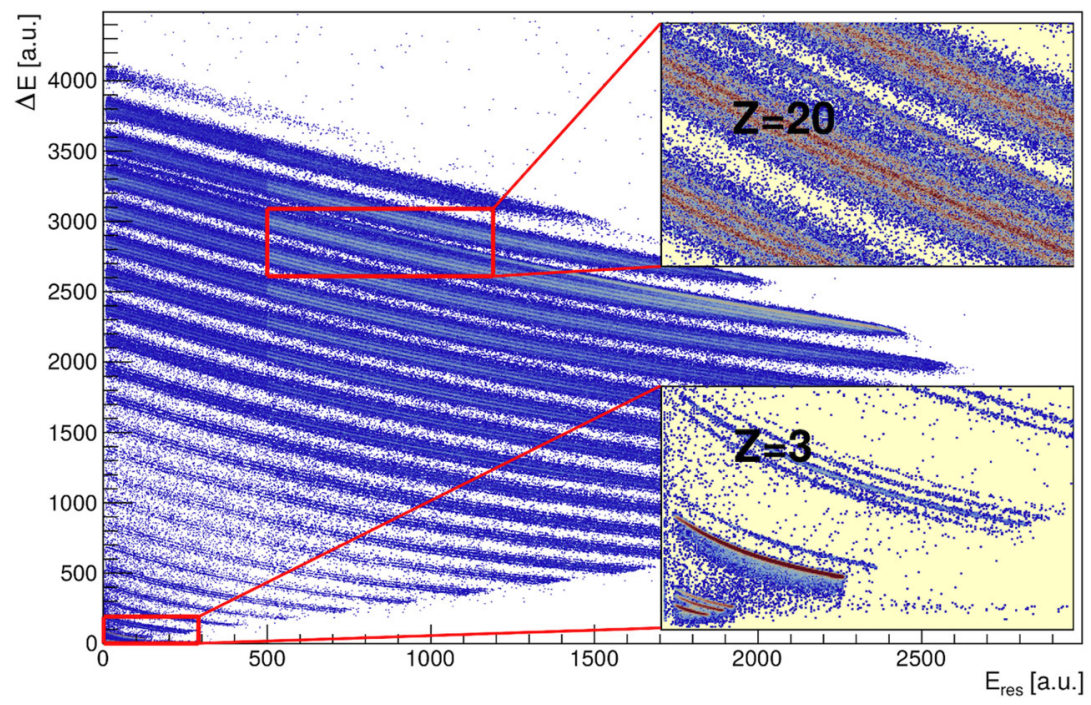

Fig. 4 NUCLEX. $\Delta E-E$ correlation in a typical FAZIA telescope (first vs second silicon layer). Data come from ${ }^{48} \mathrm{Ca}+{ }^{12} \mathrm{C}$ reactions at $25 \mathrm{MeV} /$ nucleon. The insets show the mass discrimination performances at low $(Z=3)$ and relatively high $(Z=20)$ atomic number, the ridges corresponding to the different masses being still well separated

In contrast, in a system like FAZIA with solid-state detectors as first layers, 10 to $20 \mathrm{MeV} /$ nucleon particle energies (depending on their charge) are necessary to punch through and thus allow for the use of $\Delta E-E$ method. In this case isotopic discrimination is achieved for ions up to $Z \sim 25$ ( $\Delta \mathrm{A} / \mathrm{A} \sim 2 \%, \Delta \mathrm{Z} / \mathrm{Z}<2 \%$ ), see Fig. 4. Moreover, the use of $10 \mathrm{~cm} \mathrm{CsI(Tl)}$ crystals permits the identification of very fast particles (e.g. protons up to $\sim 195 \mathrm{MeV}$ ).

Exploiting the Bethe-Bloch formula it is also possible to carry out Bragg spectroscopy in TPCs (Time Projection Chambers) such as ACTAR demonstrator [73]. In TPCs, it is possible to independently measure the energy released in small regions of the active gas volume, so the energy loss profile (Bragg curve) of the detected particles can be reconstructed and thus they can be identified by a direct comparison to simulated curves. If the particles escape from the gas volume they can be still identified via $\Delta E-E$ method, using ancillary detectors as a second layer. In the case of the ACTAR demonstrator, the energy loss is sampled in about 80 points (depending on the trajectory of the ions inside the chamber and on their energy).

Also for the telescopes of the NUCLEX Collaboration many different identification techniques are used to identify the particles hitting the detectors besides the $\Delta E-E$ method. Taking as an example the FAZIA setup, the $\Delta E-E$ technique is used when particles punch-through the first silicon layer. Otherwise, the PSA in silicon (see Sect. 4.2.3) is applied. Fast light particles are identified thanks to PSA in CsI(TI) (see Sect. 4.1.2), while slow light particles can be identified also thanks to the $E$-TOF approach (see Sect. 6.2.5). Kinematic methods (see Sect. 9.1.3) can help to associate specific reaction channels. The combination of all these techniques makes these detectors 
powerful devices able to identify nuclei over a very wide interval of energies and species.

\subsubsection{NUMEN: combining $\Delta E$ and magnetic rigidity measurement}

The PID procedure adopted in MAGNEX (see Sect. 2.3.5) is based on the combination of two techniques, the $\Delta E-E$ technique and the correlation between the residual energy and the magnetic rigidity [145,146]. The 3D tracking of the ion trajectory is performed by the focal plane detector (FPD). The energy loss and the residual energy are based on a gas tracker (filled with $99.95 \%$ isobutane at pressure ranging from few mbar up to several tenths of mbar) sensitive to the energy loss of the crossing ions and a silicon wall for the measurements of their residual energy. The atomic number of the ions can be therefore identified by the standard $\Delta E-E$ technique. A typical $\Delta E-E$ correlation plot is shown in Fig. 5a together with a graphical contour selecting the neon ions. The plotted parameters are the residual energy $\left(E_{\text {resid }}\right)$, measured by the silicon detectors wall, and the total energy loss in the FPD gas section $\left(\Delta E_{\text {tot }}\right)$. The latter is corrected for the different path lengths in the gas thanks to the tracking capabilities, consequence of the wide range of horizontal angles at the focus $\left(40^{\circ} \leq \theta_{\text {foc }} \leq 72^{\circ}\right)$ [147]. For the mass reconstruction $(A)$, an innovative particle identification technique for large acceptance spectrometers, which exploits the properties of the Lorentz force, was introduced in Ref. [148]. In the experimental conditions of NUMEN interest, in which oxygen, fluorine and neon ions should be detected, the identification procedure is successfully performed using the $X_{\text {foc }}-E_{\text {resid }}$ correlation, in which $X_{\text {foc }}$ is the horizontal position measured at the focal plane. The relationship between the two quantities is approximately quadratic with a factor depending on the ratio $\sqrt{m} / q$

$$
X_{\text {foc }} \propto \frac{\sqrt{m}}{q} \sqrt{E_{\text {resid }}} .
$$

In Fig. 5 b, the correlation plot $X_{\text {foc }}-E_{\text {resid }}$ is shown for the data selected with the graphical condition on the $\Delta E_{\text {tot }}-E_{\text {resid }}$ one (see Fig. 5a). The separation between the different neon isotopes, which allows for a clear selection of the ${ }^{18} \mathrm{Ne}^{10+}$ ejectile, as indicated by the graphical contour in Fig. 5b, is evident. The achieved resolution in this case is $\Delta \mathrm{A} / \mathrm{A} \approx 1 / 300$, better than the value already reported in Ref. [148], in which straggling effects on the $E_{\text {resid }}$ parameter were more important.

In some cases, the $X_{\text {foc }}-E_{\text {resid }}$ representation is not enough to separate ions characterized by very similar $\sqrt{m} / q$ ratio, as for example the ${ }^{22} \mathrm{Ne}^{10+}$ and ${ }^{18} \mathrm{Ne}^{9+}$ ions $(\sqrt{m} / q=0.469$ and 0.471 , respectively). In these cases, a third step in the PID procedure can be performed using the time difference between the CS (superconductive cyclotron) radiofrequency periodic signal (STOP) and the timing signal coming from the silicon detectors of the FPD (START). This signal has a relative meaning connected to the different flight time of the two ions in the spectrometer. The technique allows to clearly identify the ${ }^{22} \mathrm{Ne}^{10+}$ and ${ }^{18} \mathrm{Ne}^{9+}$ ions, as demonstrated in Ref. [149].

An important feature of the MAGNEX PID technique is that it does not require absolute energy calibrations of the $E_{\text {resid }}$ and $\Delta E_{\text {tot }}$ measurements, since they are used only for particle identification purposes. Usually, the loci in the PID plots are identified 

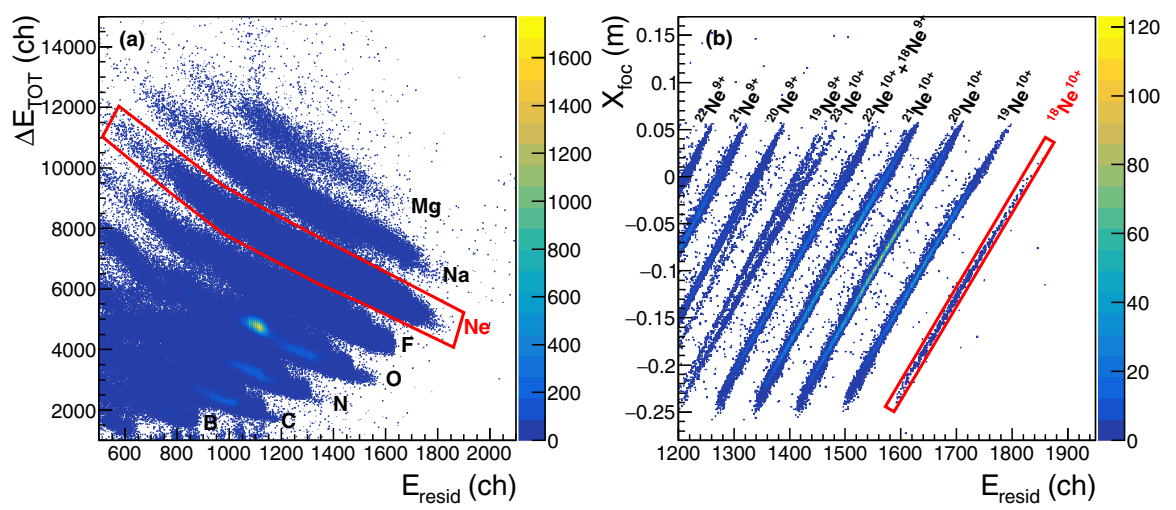

Fig. 5 NUMEN. PID technique developed for experiments performed with the MAGNEX spectrometer. a Typical $\Delta E_{\text {tot }}$ vs. $E_{\text {resid }}$ plot for unselected events in a single silicon detector measured in the ${ }^{18} \mathrm{O}+{ }^{76} \mathrm{Se}$ reaction at $275 \mathrm{MeV}$ and $0^{\circ} \leq \theta_{\text {lab }} \leq 8^{\circ}$. The different atomic species and a graphical contour on the neon region are indicated (red line). b Typical $\mathrm{X}_{\text {foc }}$ vs. $E_{\text {resid }}$ plot for the selected neon events on the $\Delta E_{\text {tot }}$ vs. $E_{\text {resid }}$ for the same silicon detector (a) zoomed in the region of the $10^{+}$charge state. The different neon isotopes and a graphical contour (red line) selecting the ${ }^{18} \mathrm{Ne}^{10+}$ are indicated

through supplementary elastic scattering runs. The capability of the PID in terms of the minimum cross-section significantly measurable by the MAGNEX spectrometer was investigated [150], demonstrating that it is suitable for accurate measurements of very rare processes like double charge exchange reactions, characterized by cross sections as low as tens of nbarn.

\subsection{ALICE: the high-energy limit for particles and light nuclei at the GeV scale}

In the ALICE experiment, most of the detected particles by means of energy loss measurement like baryons, mesons and nuclei are MIP's. In this framework, PID is performed by the Inner Tracking System (ITS) and the Time Projection Chamber (TPC) correlating the energy loss with the momentum measured thanks to the magnetic field of ALICE. The ITS consists of six cylindrical layers of silicon detectors. It covers the pseudorapidity range $|\eta| \leq 0.9$ and it is the central barrel detector closest to the beam axis. The distance from the nominal beam line ranges from $3.9 \mathrm{~cm}$ for the innermost layer up to $43 \mathrm{~cm}$ for the outermost. The two innermost layers are made of silicon pixel detectors (SPD), the two central layers of silicon drift detectors (SDD) and the two outermost layers of double-sided silicon strip detectors (SSD).

A detailed description of the three sub-systems can be found in [40]. The ITS has the main purposes of providing both primary and secondary vertices reconstruction and of improving the ALICE barrel tracking capabilities in the vicinity of the interaction point. The four outer layers (SDD and SSD) have analogue readout and therefore can be used for particle identification via $\mathrm{d} E / \mathrm{d} x$ measurement in the non-relativistic region. The analogue readout has a dynamic range large enough to provide the $\mathrm{d} E / \mathrm{d} x$ measurement for low-momentum ionising particles, down to the lowest momentum at which tracks can still be reconstructed. This feature gives the ITS stand-alone capability as a low- $p_{\mathrm{T}}$ particle spectrometer. 

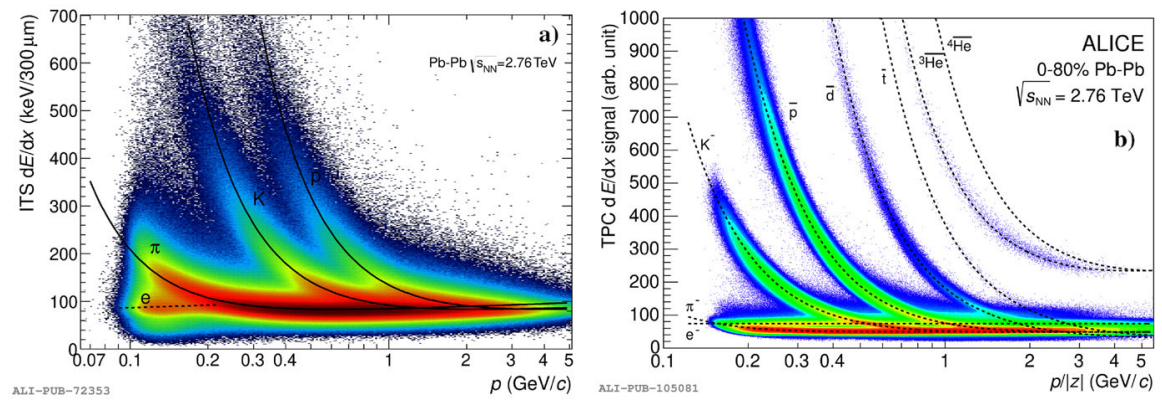

Fig. 6 ALICE. a Distribution of the energy-loss signal in the ITS as a function of momentum in $\mathrm{Pb}-\mathrm{Pb}$ collisions at $\sqrt{s_{\mathrm{NN}}}=2.76 \mathrm{TeV}$ (from [41]). Curves represent the Bethe-Bloch parameterisation. b distribution of specific energy loss $\mathrm{d} E / \mathrm{d} x$ in the TPC as a function of the rigidity $(p /|Z|)$ for negative particles in $\mathrm{Pb}-\mathrm{Pb}$ collisions at $\sqrt{s_{\mathrm{NN}}}=2.76 \mathrm{TeV}$. The dashed lines are parameterisations of the BetheBloch formula for the different particle species (from [152])

The ALICE SDDs were produced from very homogeneous high-resistivity (3 $\mathrm{k} \Omega \mathrm{cm}$ ) 300- $\mu \mathrm{m}$-thick Neutron Transmutation Doped (NTD) silicon, with a sensitive area of $70.17(r \phi) \times 75.26(z) \mathrm{mm}^{2}$ and a total area of $72.50 \times 87.59 \mathrm{~mm}^{2}$. The sensitive area is split into two drift regions by the central cathode strip to which a HV bias of $-2.4 \mathrm{kV}$ is applied. In each drift region, and on both detector surfaces, $291 \mathrm{p}^{+}$ cathode strips, with $120 \mu \mathrm{m}$ pitch, fully deplete the detector volume and generate a drift field parallel to the wafer surface. A SDD module consists of one silicon drift detector and two front-end hybrids, each connected to the corresponding end-ladder low-voltage board. The two layers of SSD are crucial for the matching of tracks from the TPC to the ITS. Minimization of the material budget of the mechanical support for the detection modules is achieved using linear Carbon Fibre Composite (CFC) material for all support structures in the active volume. The sensors have 768 strips on each side with a pitch of $95 \mu \mathrm{m}$. The stereo angle is $35 \mathrm{mrad}$ which is a compromise between stereo view and reduction of ambiguities resulting from high particle densities. The stereo angle is obtained by defining strips with an angle of $7.5 \mathrm{mrad}$ with respect to the sensor short edge on the p-side and with an angle of $27.5 \mathrm{mrad}$ on the n-side.

The stand-alone tracking in the ITS favors a momentum range wider towards lower $p_{\mathrm{T}}$ values, while the combined tracking with TPC provides a better momentum resolution. The energy loss measurement in each layer of the ITS is corrected for the track length in the sensitive volume using tracking information. In the case of SDD clusters, a linear correction for the dependence of the reconstructed raw charge as a function of drift time due to the combined effect of charge diffusion and zero suppression is also applied [151]. For each track, the energy loss is evaluated using a truncated mean. The average of the lowest two points in case four points are measured, or a weighted sum of the lowest (weight 1 ) and the second lowest point (weight $1 / 2$ ), in case only three points are measured, is done. The final $\mathrm{d} E / \mathrm{d} x$ resolution is about $10 \%$.

An example distribution of measured truncated mean energy loss values as a function of momentum in the ITS is shown in Fig. 6a. By means of this technique it is 
possible to identify $\pi^{ \pm}, \mathrm{K}^{ \pm}$, and $(\overline{\mathrm{p}}) \mathrm{p}$ in $\mathrm{Pb}-\mathrm{Pb}(\mathrm{pp})$ collisions in the $p_{\mathrm{T}}$ intervals $0.1-0.7 \mathrm{GeV} / c, 0.2-0.5(0.6) \mathrm{GeV} / c$, and $0.3-0.6$ (0.65) $\mathrm{GeV} / c$, respectively.

In ALICE, identification via energy loss is completed by the TPC detector [153] which has a gas drift volume of $90 \mathrm{~m}^{3}$ and allows identification of positive and negative pions, kaons and protons within the transverse momentum intervals $0.25-0.70 \mathrm{GeV} / c$, $0.25-0.45 \mathrm{GeV} / c$, and $0.45-0.90 \mathrm{GeV} / c$, respectively, and from $p_{\mathrm{T}} \approx 2-3 \mathrm{GeV} / c$ up to $p_{\mathrm{T}}=20 \mathrm{GeV} / c$ exploiting the $\mathrm{d} E / \mathrm{d} x$ signal in the relativistic rise region $(3<\beta \gamma<<1000)$. An example of the distribution of energy loss values as a function of momentum in the TPC is shown in Fig. 6b. Nuclei like deuteron, tritium and ${ }^{3} \mathrm{He}$ are clearly identified in the $p_{\mathrm{T}}$ range $0.7-1.4 \mathrm{GeV} / c, 0.6-2 \mathrm{GeV} / c, 2-7 \mathrm{GeV} / c$, respectively. Identification of ${ }^{4} \mathrm{He}$ is possible exploiting also the time of flight information (see Sect. 6.1.1). Both the ITS and TPC are being upgraded for the 2022 data taking [154].

\subsection{FOOT: particle identification for light fragment cross section measurements with emulsions}

As mentioned in Sect. 2.5.1, in the FOOT experiment an emulsion spectrometer was developed for the identification of light fragments. It is divided into three parts:

- vertex and tracking detector, which is made of elementary cells of carbon or $\mathrm{C}_{2} \mathrm{H}_{4}$ layers, acting as target, alternated with emulsion film $(300 \mu \mathrm{m})$ to track the secondary fragments and reconstruct the vertex position.

- ionization detector for charge identification, composed of elementary cells, containing three emulsion films. After data taking, they are treated at different temperatures to separate low $Z$ fragments $(\mathrm{H}, \mathrm{He}, \mathrm{Li})$.

- tracking detector for momentum measurements, using $300 \mu \mathrm{m}$ thick emulsion films interleaved with 1-mm-thick lead plates as passive material. The mass and momentum can be obtained using two independent measurements (range and multiple Coulomb scattering).

The emulsion setup has been built and tested during various test beams, demonstrating an excellent performance in charge identification [155]. An example of the performance can be seen in Fig. 7, where the separation between two different populations of ions ( ${ }^{4} \mathrm{He}$ in red and ${ }^{12} \mathrm{C}$ in green) can be seen [156]. The Volume Refreshing (VR) parameters on the axes are an estimate of the ionization $(\mathrm{V})$ produced in the nuclear emulsions after two different thermal treatments (VR1 and VR2). The resolution on the charge measurement for ${ }^{4} \mathrm{He}$ and ${ }^{12} \mathrm{C}$ ions was about $8 \%$.

\section{Pulse shape analysis (PSA) for charged particles, neutrons and $\gamma$-rays}

In this section PID techniques based on the PSA are presented. In these methods the particle identification is achieved from the correlation between two parameters related to the shape, i.e. to the evolution as a function of time of the signal induced by the detection of a particle, exploiting the fact that fragments with different $Z, A$ 


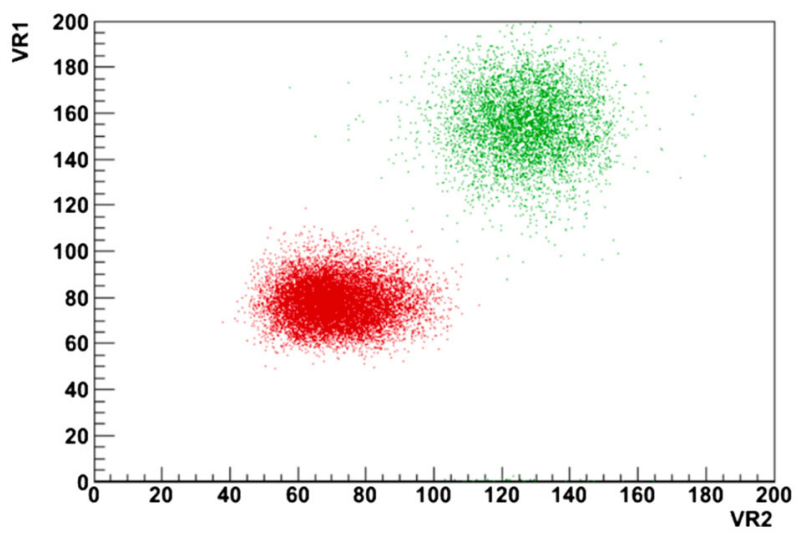

Fig. 7 FOOT. Scatter plot showing two different populations of ions ( ${ }^{4} \mathrm{He}$ in red and ${ }^{12} \mathrm{C}$ in green), separated according to their ionization produced in the nuclear emulsions. VR1 and VR2 are the track volumes normalized with respect to the emulsion thickness for two different thermal treatments (from [156])

and equal incident energy $E$ produce signals of different profiles in the detector. The main advantage of this technique is the need of only one detector layer to obtain the particle identification, thus allowing a significant reduction of the detection thresholds with respect to the case of the $\Delta E-E$ technique. Since many years the method is widely used for scintillators to identify light charged particles but also to discriminate neutrons and $\gamma$; more recently the technique has been applied also to silicon detectors to identify in charge (up to heavy ions, $Z \sim 50$ ) and mass (up to medium size ions, $Z \leq 20)$ the reaction ejectiles. The identification threshold depends on the range of the ion in silicon and it increases with the charge of the ion (for example, the minimum range is around $40 \mu \mathrm{m}$ for $\mathrm{B}$ and around $60 \mu \mathrm{m}$ for $\mathrm{Ne}$ ). The charge being the same, the isotopic identification is more demanding and the minimum required range increases (for example, the charge identification for Carbon has a range threshold of $40 \mu \mathrm{m}$, while its isotopic identification requires a minimum range greater than $100 \mu \mathrm{m}$ ).

A different kind of PSA is that related to the $\gamma$-ray tracking in segmented HPGe detectors. In this case, the goal is to improve the energy resolution of the array by reconstructing the interaction positions of the $\gamma$ rays inside the crystals. This can be achieved by comparing the acquired signals with a library of shapes obtained once and for all illuminating the detector with collimated sources of known position.

\subsection{PSA technique in CsI(TI) scintillators}

Thallium-doped cesium iodide scintillators are commonly used as the last stage detectors in telescopes because of their high stopping power, the good energy resolution and the ease of machining and operation.

A big advantage of $\mathrm{CsI}(\mathrm{Tl})$ detectors is the possibility to perform PSA for detected particles. This possibility comes from the fact that the scintillation of the CsI(Tl) crystal is well described by the sum of two exponentials with different time constants: a short one $\left(\tau_{\mathrm{s}} \sim 0.75 \mu \mathrm{s}\right)$ and a long one $\left(\tau_{1} \sim 5 \mu \mathrm{s}\right)$. If we call $Q_{\mathrm{s}}$ and $Q_{1}$ the 
charges induced in the photodiode/photomultiplier by short and long components of scintillation, respectively, particle identification capability comes from the different ionization density dependence of $Q_{\mathrm{s}}$ and $Q_{1}$ components on $Z$ and $A$ of the ejectiles (i.e. of the reaction products). In particular, with the same total energy, the short scintillation component (commonly called "fast" component) is more intense for highly ionizing particles, i.e. with greater $Z$ and $A$. On the other side, the long component (commonly called "slow" component) is more intense for $\gamma$ and cosmic rays, because they produce small ionization densities. ${ }^{2}$ By means of two shapers with different shaping constants (sometimes applied via software on the digitized signal) it is possible to determine the intensity of the two components and so to perform PSA, identifying in charge and mass light charged particles stopped inside the crystal, thanks to the correlation between the fast and the slow components.

\subsubsection{CHIRONE}

Both CHIMERA and FARCOS multidetectors (see Sect. 2.3.1) are equipped with CsI(Tl) scintillators. For FARCOS the CsI(Tl) have a thickness of $6 \mathrm{~cm}$ and their area is $3.2 \times 3.2 \mathrm{~cm}^{2}$, while for CHIMERA the thickness ranges from $10-12 \mathrm{~cm}$ in the forward part to $8-3 \mathrm{~cm}$ in the backward part, with an area of $5 \times 5 \mathrm{~cm}^{2}$.

For the CHIMERA multidetector the pulse shape discrimination, based on the two gates method [157], is used to identify the light charged particles stopped in CsI(Tl) scintillators. This method permits an isotopic separation for particles with $Z<5$. The same method is used with CsI(Tl) scintillators of the FARCOS array. Recently, also a digital electronics (GET) can be used, that allows the digitization of the total shape of signals $[158,159]$.

Figure 8 shows a fast-slow correlation obtained in the reaction ${ }^{124} \mathrm{Xe}+{ }^{64} \mathrm{Ni}$ at $35 \mathrm{MeV} /$ nucleon in a FARCOS CsI(Tl) module. The inset shows the PID spectrum, obtained tracing lines on each ridge corresponding to a different particle species, where $\mathrm{H}, \mathrm{He}$ and $\mathrm{Li}$ isotopes are easily distinguished.

\subsubsection{NUCLEX}

GARFIELD, RCo and FAZIA setups (see Sects. 2.3.4 and 3.2.2) are equipped with $\mathrm{CsI}(\mathrm{Tl})$ detectors; the thickness of the CsI crystal has been chosen to stop even the most energetic light charged particles in the typical reactions investigated by NUCLEX, i.e. protons up to $105 \mathrm{MeV}$ in GARFIELD + RCo and up to $195 \mathrm{MeV}$ in FAZIA. In GARFIELD, RCo and in FAZIA these different shaping operations have been implemented directly on the digital electronics card purposely designed for these setups $[70,160]$. By correlating the ratio between fast and slow shaped energy values versus the slow one it is possible to discriminate $\gamma$ rays and all the hydrogen, helium and lithium (in some cases even Be) isotopes (Fig. 9). Unfortunately, heavier fragments tend to overlap to the same ridge in the correlation, but they can be easily discriminated with $\Delta E-E$ technique using the $\mathrm{CsI}(\mathrm{Tl})$ layer as the stopping layer (residual energy $E_{\text {res }}$ detector).

\footnotetext{
2 In the $\mathrm{CsI}(\mathrm{Tl})$ the smaller the ionization density the slower the signal.
} 


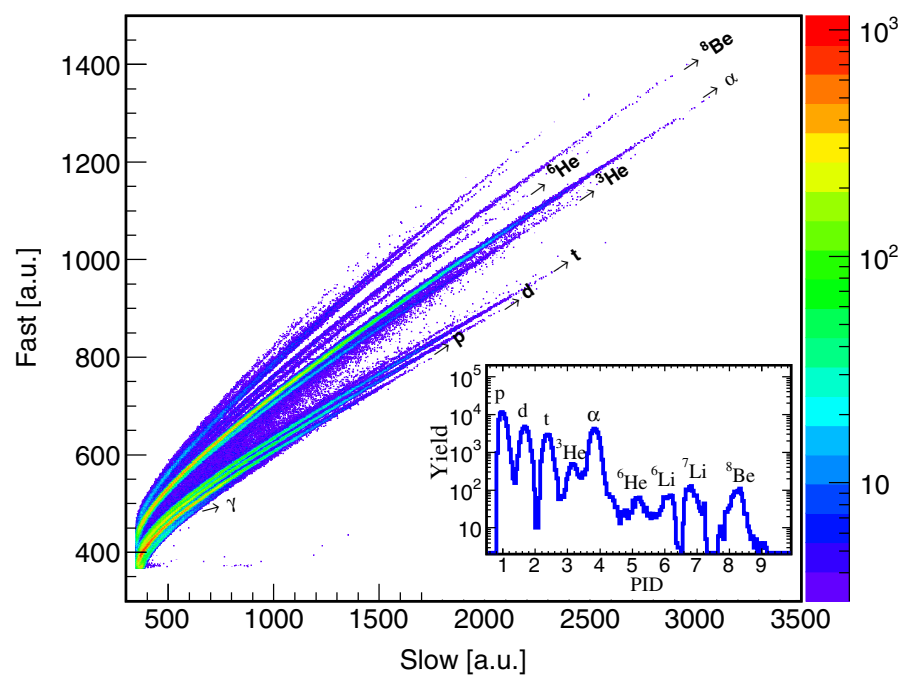

Fig. 8 CHIRONE. Fast-slow correlation obtained with a CsI(Tl) of FARCOS array, for the reaction ${ }^{124} \mathrm{Xe}$ $+{ }^{64} \mathrm{Ni}$ at $35 \mathrm{MeV} /$ nucleon. The inset shows the yield as a function of the PID. For $\alpha$ particles the FWHM is 0.26

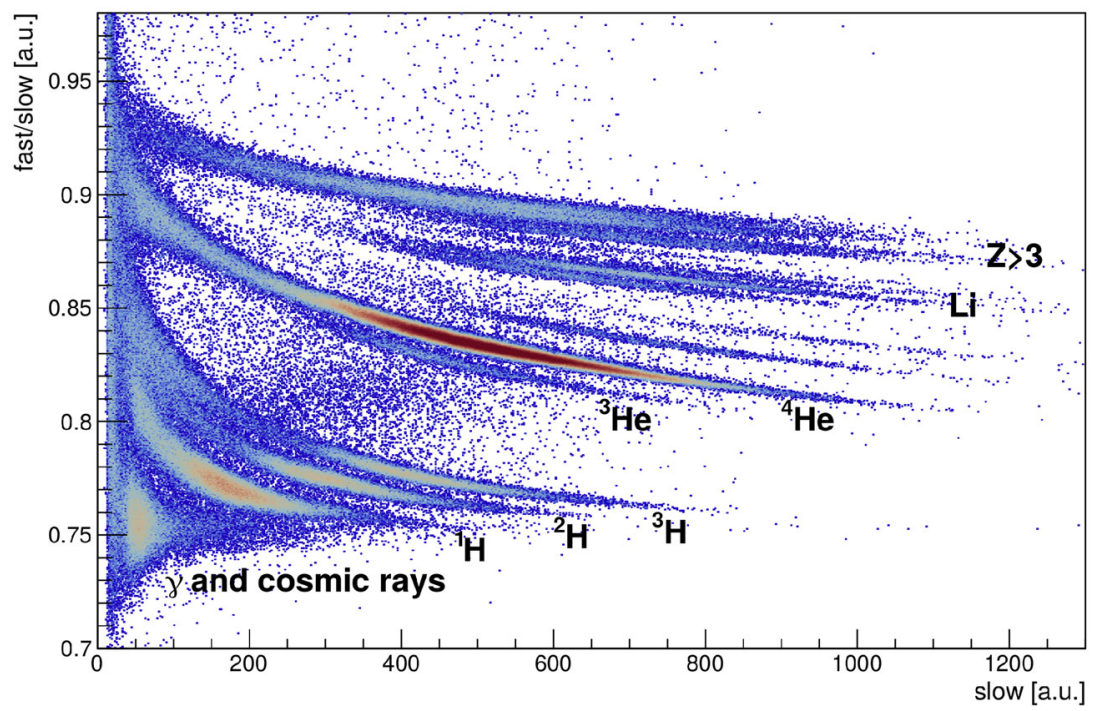

Fig. 9 NUCLEX. Fast/slow vs slow correlation in a typical FAZIA CsI(Tl) crystal. Hydrogen, helium and lithium isotopes are well discriminated. Data come from ${ }^{48} \mathrm{Ca}+{ }^{12} \mathrm{C}$ reactions at $25 \mathrm{MeV} /$ nucleon

There are some limits of the PSA technique in CsI(Tl) scintillators: first, neutrons may interact with the crystal via $(\mathrm{n}, \mathrm{p})$ or $(\mathrm{n}, \alpha)$ reactions and thus they appear overlapped to protons or $\alpha$ particles in the correlation; second, protons (especially with energy above $100 \mathrm{MeV}$ ) can experience interactions (Coulomb or nuclear elastic scattering and reactions) which, in some cases, produce events characterized by an incomplete 
energy deposition (IED). In this kind of events, protons deviate off the $Z=1$ region, moving to the $\gamma$-like ridge in the correlation [161]. This is due to the fact that protons, in IED, react or escape from the crystal volume before reaching the Bragg peak, and thus they produce a track with moderate ionization density in the crystal, mainly stimulating the long scintillating component.

The experiments performed by NUCLEX collaboration are focused on charged particles, thus it is important to distinguish and possibly reject $\gamma$ particles and neutrons. Silicon layers in front of $\mathrm{CsI}(\mathrm{Tl})$ crystals have an almost zero detection efficiency for chargeless radiation, thus $\Delta E-E$ correlations involving Si pads automatically reject $\gamma$ rays and neutrons. Si pads are used also as a veto to reject those kinds of particles from PSA in CsI(Tl) scintillators. As a general rule, since many identification mismatches (due to neutrons interacting with $\mathrm{CsI}(\mathrm{Tl})$ and producing $\mathrm{n}$ or $\alpha$ ) are possible with PSA in scintillators, the PID priority should be given to the $\Delta E-E$ method. Instead, the case of IED is much more complex to handle, because usually fast protons release very little energy in Si layers and sometimes it is impossible to identify them via $\Delta E-E$ technique.

\subsection{PSA in silicon detectors}

The PSA in Si detector has been studied and developed during the last thirty years [162166]. In these detectors, the different shapes of the signals associated with different ions, being equal their energy, are due to the variation with $Z, A$ and $E$ of the penetration depth and of the ionization density profile along the particle track, which in turn affect the plasma erosion time [167,168] and the collection time of the electron-hole pairs. The technique is able to identify in charge up to heavy ions $(Z \sim 50)$ and in mass up to medium size ions $(Z \sim 20)$.

\subsubsection{CHIRONE}

The collaboration has proven the capabilities of CHIMERA multidetector to provide accurate results in the Fermi energy regime, characterized by final states with a large number of charged products that populate a broad energy range. However, the possibility to employ the pulse shape analysis in Si detectors expanded the energy regime towards lower energy ( $E \leq 10 \mathrm{MeV} /$ nucleon) [169]. In CHIMERA, the PSA in Si is based on the analysis of the rise time of Si signals, and it allows the identification in charge of slow-moving particles, stopped in Si detectors (with a thickness of $300 \mu \mathrm{m}$ ), having $3 \leq \mathrm{Z} \leq 18$ [46,170]. With the CHIMERA multidetector this technique is applied in Si direct configuration (i.e. entrance from the high electric field side), not to spoil time of flight measurements. In fact, a good time of flight requires fast signals and this can be achieved if the particles experience a high electric field at the entrance in the detector. The energy threshold for the charge identification ranges from 4 to $9 \mathrm{MeV} /$ nucleon, as a function of increasing detected charge. The first experiment performed using the PSA in Si in a low-energy regime was ISODEC [169], studying the isospin influence on compound nucleus decay modes in the reactions ${ }^{78} \mathrm{Kr}+{ }^{40} \mathrm{Ca}$ and ${ }^{86} \mathrm{Kr}+{ }^{48} \mathrm{Ca}$ at $10 \mathrm{MeV} /$ nucleon. Figure $10 \mathrm{a}$ shows an energy-rise time correla- 

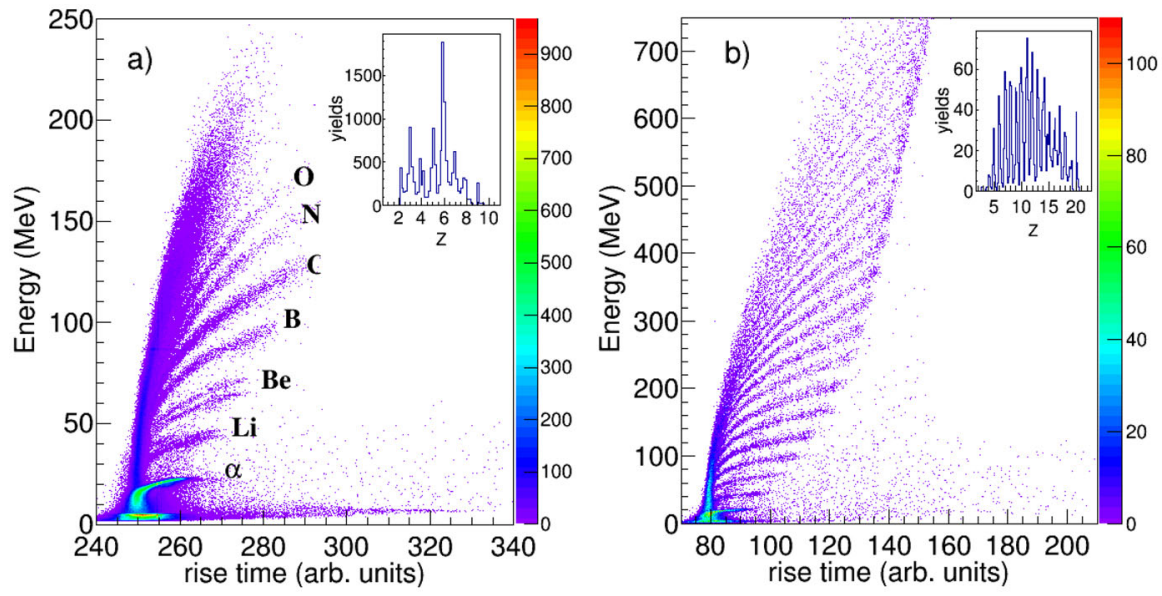

Fig. 10 CHIRONE. a Energy of particles stopped in silicon detectors as a function of the signal rise time, at around $42^{\circ}$, for ${ }^{78} \mathrm{Kr}+{ }^{40} \mathrm{Ca}$ reaction at $10 \mathrm{MeV} /$ nucleon. The inset shows the $Z$ distribution determined using the energy-rise time plot, after excluding the region with short rise time where charge identification is not achieved. $\mathbf{b}$ The same as in a but at around $15.25^{\circ}$, for ${ }^{124} \mathrm{Xe}+{ }^{64} \mathrm{Zn}$ reaction at $35 \mathrm{MeV} /$ nucleon. The inset shows the $Z$ distribution determined using the energy-rise time plot, after excluding the region with short rise time where charge identification is not achieved

tion obtained in the first reaction: the inset shows the $Z$ distribution determined using the energy vs. rise-time plot. However, this method is also extensively used at higher energy, as we can see in Fig. 10b that shows an energy vs. rise-time plot obtained in the reaction ${ }^{124} \mathrm{Xe}+{ }^{64} \mathrm{Zn}$ at $35 \mathrm{MeV} /$ nucleon [46]. The energy threshold for the charge identification depends on $\mathrm{Z}$; for example, it corresponds to $5.6 \mathrm{MeV} /$ nucleon for $Z=6$ and it increases to $9 \mathrm{MeV} /$ nucleon for $Z=16$.

\subsubsection{GAMMA}

GAMMA exploits the PSA in silicon detectors in the GRIT (Granularity Resolution Identification Transparency) array $[171,172]$ and in the GALTRACE (GALILEO TRacking Array for Charged Ejectiles) prototype to identify light particles in charge and mass.

GRIT is an array of segmented silicon detectors developed within an international collaboration with the aim of performing particle spectroscopy and discrimination. It is designed to work as a standalone device or combined to large $\gamma$ spectrometers. It consists of 48 detectors in telescopic $\Delta \mathrm{E}$-E configuration arranged in a sphere-like geometry. For each telescope, the first $(\Delta E$ ) layer is a 500- $\mu$ m-thick nTD (neutrontransmutation doped) detector while the second layer $(E)$ is $1.5 \mathrm{~mm}$ thick. The detectors are segmented in 128 strips on both sides.

A small prototype detector array, GALTRACE, was recently developed to optimize the electronics. The detector readout is performed with custom ASIC (ApplicationSpecific Integrated Circuit) pre-amplifiers [173-176]. The energy information is obtained with trapezoid filters using MWD (moving window deconvolution) implemented on the acquisition FPGAs (Field-Programmable Gate Arrays). 


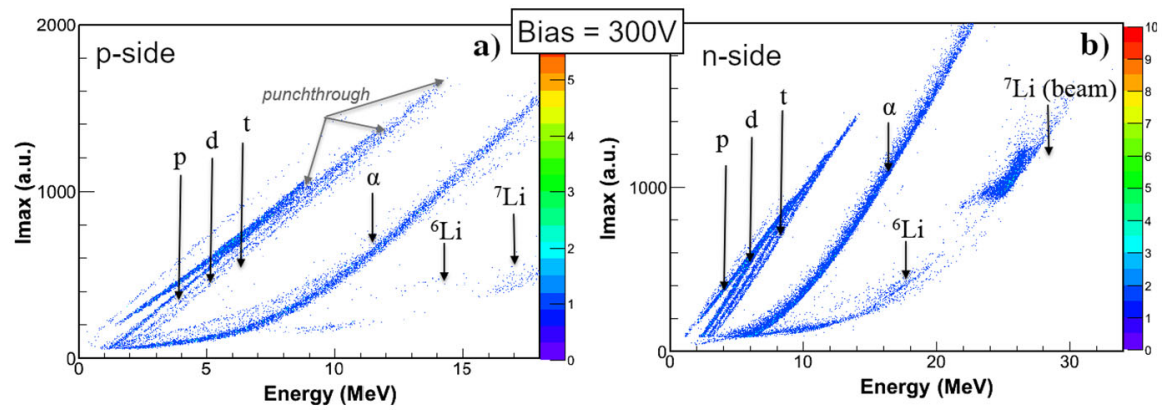

Fig. 11 GAMMA. Maximum signal derivative versus total amplitude, performed on one GRIT detector's p-side strip (a) and one n-side strip (b). From [179]

By analyzing the characteristics of the pre-amplifier signal leading edge, it is possible to gain information about the particles impinging on the detector. Clear PID can be obtained plotting the rise time or the maximum of the signal derivative (Imax) versus the deposited energy of the particles (see Fig. 11). This technique, complementary to the $\Delta E-E$ one, allows to extend the particle discrimination capabilities of the detectors to particles stopping in the first silicon layer [177], thus allowing to cover the low-energy region, down to $\sim 1 \mathrm{MeV} /$ nucleon for the main light particles $[178,179]$.

Further developments of an integrated front-end electronics with reduced power consumption and the inclusion of the analog on-line signal are in progress. An analog memory $[180,181]$ is currently under development within the GRIT collaboration. Its aim is to work as an efficient, low-noise, low-power multiplexing system for the analog signals inside the reaction chamber.

\subsubsection{NUCLEX}

FAZIA collaboration widely studied PSA in silicon detectors, also developing a microscopic [182] and a phenomenological [165] model, which consider the progressive extraction of the electrons and holes from the high carrier density zone along the ionizing particle track. See [69] for a comprehensive report of all the work on PSA carried out by the collaboration.

There are two ways to analyze the pulse shape: it is possible to measure the rise time of the charge signal or the maximum value of the current pulse; then the correlation of the chosen variable to the energy released inside the sensor is built. In this way, ridges corresponding to different atomic species can be obtained, and, in optimized detectors, also isotopes for the lightest and most energetic fragments can be discriminated. The PSA performance is adversely affected by various factors like channeling effects, range straggling, non-uniformity of doping, residual sheet resistance and instability of the applied bias (see [69] for more details). For this reason, during FAZIA R\&D phase, much effort has been devoted to the optimization of the silicon sensors:

- wafers are obtained from ingots with excellent declared doping homogeneity, usually but not uniquely guaranteed by the use of nTD technique; 


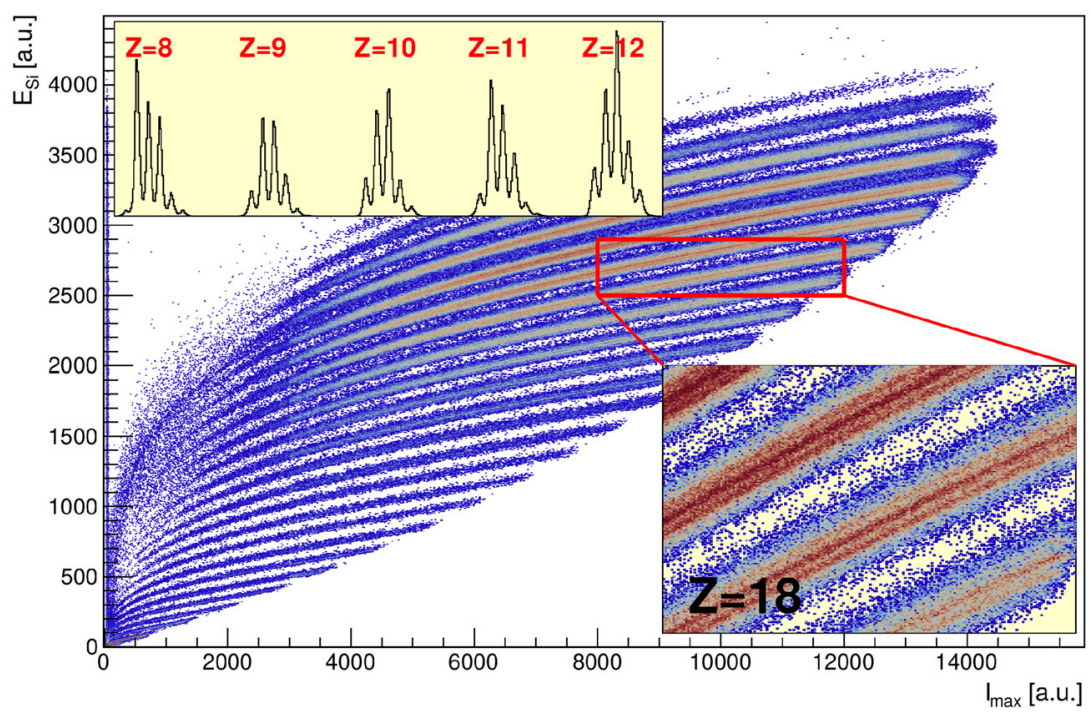

Fig. 12 NUCLEX. $E-I_{\max }$ correlation in a typical FAZIA first silicon layer. Isotope discrimination is achieved up to $Z=20$. In the upper inset the PID spectrum in the region $Z=8-12$, restricted to the zone of the correlation in which the isotopic separation is achieved, is shown. In the bottom inset the region around $Z=18$ is expanded. Data come from ${ }^{48} \mathrm{Ca}+{ }^{12} \mathrm{C}$ reactions at $25 \mathrm{MeV} /$ nucleon

- wafers are cut from ingots with an angle of $\sim 7^{\circ}$ with respect to the main crystal axis to reduce channeling effects;

- pads are covered with a thin metallization layer to reduce the sheet resistance;

- bias voltage is kept under control and adjusted in real time to compensate for the drop due to the increasing (usually with radiation) reverse current circulating through the bias resistors.

With such treatments it is possible to distinguish isotopes for ions up to $Z \sim 20$ with a threshold of around $10 \mathrm{MeV} /$ nucleon (mass resolution about $5 \%$ ), while charge identification is possible from 2-5 MeV/nucleon depending on the fragment atomic number (Fig. 12), with charge resolution about $2 \%$.

Of course, there are limits in the applicability of PSA in detectors when the particle tracks are modestly penetrating in the active volume (i.e. towards low deposited energies). For example, according to studies of the FAZIA group [183] charge identification for light ions in silicon is prevented for ion range below $30 \mu \mathrm{m}$. In the same paper the quality of PSA is compared between front mounted (i.e. particles entering through the high electric field side) and rear-mounted detectors (i.e. particles entering through the low electric field side): the study shows that the identification threshold is much lower in the latter case, while there is no impact on energy resolution (and thus on the complementary $\Delta E-E$ technique). For this reason, RCo (see Sect. 3.2.2) and FAZIA (see Sect. 3.2.2) adopt rear mounting.

PSA strongly benefits of digital processing: all the achievements obtained with RCo and FAZIA in lowering identification thresholds were possible also thanks to the digital electronics these detectors are equipped with. In fact, both in GARFIELD + RCo and 
in FAZIA, after a low-noise analogue stage including preamplifier and antialiasing filter, signals are immediately sampled with fast and high-resolution ADCs (14-bit, $250 \mathrm{MHz}$ in FAZIA). As shown in [184], a proper digital processing of the sampled signals permits to improve the PSA quality.

\section{3 $n-\gamma$-Charged particles and/or $n-\gamma$ discrimination}

The discrimination among neutrons and $\gamma$ and/or charged particles is generally achieved by means of the PSA in various kinds of scintillators (liquid, solid, organic, inorganic), mainly correlating the fast vs. slow component as in the case of CsI(Tl) crystals (see Sect. 4.1). In nuclear astrophysics experiments the $n-\gamma$-charged particle discrimination is necessary to reject the background, for example that due to the intrinsic radioactivity of the detector. Neutron detection and discrimination from $\gamma$-ray is a common issue for nuclear safety and security, nuclear medicine, fundamental nuclear physics. For the latter, the neutrons can be used for the identification of the reaction products during fusion-evaporation reactions producing neutron-deficient species. On the other side of the nuclear chart, neutron emission is an important part of the decay process with $\beta$-delayed neutron emission. The two aforementioned cases required different types of detectors: for the in-beam data, fast detectors with high detection efficiency are more suitable, while for the decay studies, since neutron energy gives access to key spectroscopic information, good neutron energy measurement is the key to reconstruct the precise level scheme.

\subsubsection{CHIRONE}

In experiments with nuclear reactions induced by stable and exotic heavy-ion beams the discrimination of neutrons and light charged particles is of paramount importance. In this framework, the collaboration is carrying out a study to investigate performances of EJ 299-33 scintillator in discriminating charged particles, neutrons and $\gamma$-rays [185187]. The used method is based on the pulse shape discrimination, using the fast and slow components of the scintillator light output. The discrimination properties of the scintillator have been investigated by selecting different parts of the digitized pulse and comparing the areas under such time windows. A clear separation of neutrons from $\gamma$-rays is observed in Fig. 13a. This can be better appreciated looking at the inset, where the projection on the Slow Component axis for events belonging to the region between the two dashed lines on the Fast Component axis is shown. In Fig. 13b the discrimination between $\alpha$ particles and neutrons is shown. In this case, a ${ }^{228} \mathrm{Th} \alpha$ source and an AmBe neutron source were used. The collaboration performed also a further study employing stable beams, delivered by the Cyclotron at INFN-LNS, to investigate the performance of EJ 299-33 scintillator in experiments with heavy ions, i.e. under high background conditions [186]. The results obtained with both radioactive sources and beams are very promising and show the good capability of the EJ 299-33 to discriminate neutrons, charged particles and $\gamma$-rays. 

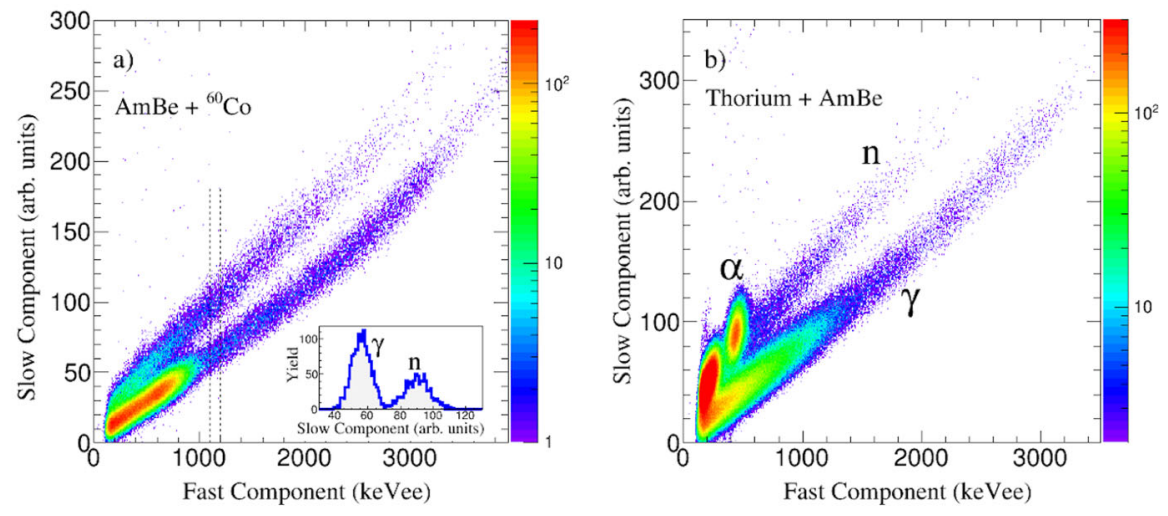

Fig. 13 CHIRONE. a The slow vs fast components plot shows neutron- $\gamma$ discrimination, in EJ 299-33 scintillator irradiated with $\mathrm{AmBe}$ and ${ }^{60} \mathrm{Co}$ sources. The inset shows the Figure of Merit (FOM) for neutron$\gamma$ separation relative to the slice in the fast component (1100-1200 keVee) displayed with dashed lines. Figure adapted from Ref. [185]. b The plot shows $\alpha$, neutron and $\gamma$-ray discrimination obtained using ${ }^{228} \mathrm{Th}$ and AmBe sources. The unit keVee is for $\mathrm{keV}$ electron equivalent [185]

\subsubsection{GAMMA}

GAMMA exploits the $n-\gamma$ discrimination both for spectroscopy (using inorganic scintillators in the PARIS setup [188] and investigating modern materials as the "CLYC": $\mathrm{Cs}_{2} \mathrm{LiYCl}_{6}: \mathrm{Ce}$ ) and for in-beam experiments (using organic scintillators in the NEDA array).

Scintillators designed to detect neutrons in in-beam experiments, such as the NEDA array [189], must present the largest possible detection efficiency for fast neutrons. NEDA consists of detection modules of organic liquid scintillator (EJ-301). Designed to be coupled with the AGATA array, NEDA makes use of a digital electronics developed within the collaboration. Because of the large counting rate in the liquid scintillators, due to the large $\gamma$ background in fusion-evaporation experiment $(\sim 80 \mathrm{kHz}$ / NEDA cell), a first level of neutron- $\gamma$ discrimination needs to be performed online. For this purpose, two pieces of information can be combined at the FPGA level: a standard charge-comparison (CC) algorithm and a time-of-flight measurement using an external reference signal. Charge-comparison algorithms rely on the comparison between two integration windows of the signal, generally one short ( $\sim 20-50 \mathrm{~ns})$ and one long $(\sim 250 \mathrm{~ns})$. This is effective, since signals generated by neutron events have a longer tail respect to the ones induced by $\gamma$-rays. Neutron selection can be configured on a detector-by-detector basis. Typical neutron- $\gamma$ discrimination figure are presented in Fig. 14, where the CC algorithm result is correlated to the total charge (panel a) and the TOF (panel $b$ ) for in-beam data obtained during the AGATA-NEDA campaign of 2018. The FPGA selection allows to reduce the $\gamma$ background, thus allowing large beam intensity resulting in very high counting rates.

On the other hand, scintillators designed to provide spectroscopic information should measure, with the highest possible precision, (i) the energy of the incident radiation, (ii) the interaction instant, and (iii) the nature of the radiation (e.g., $\gamma$-rays 

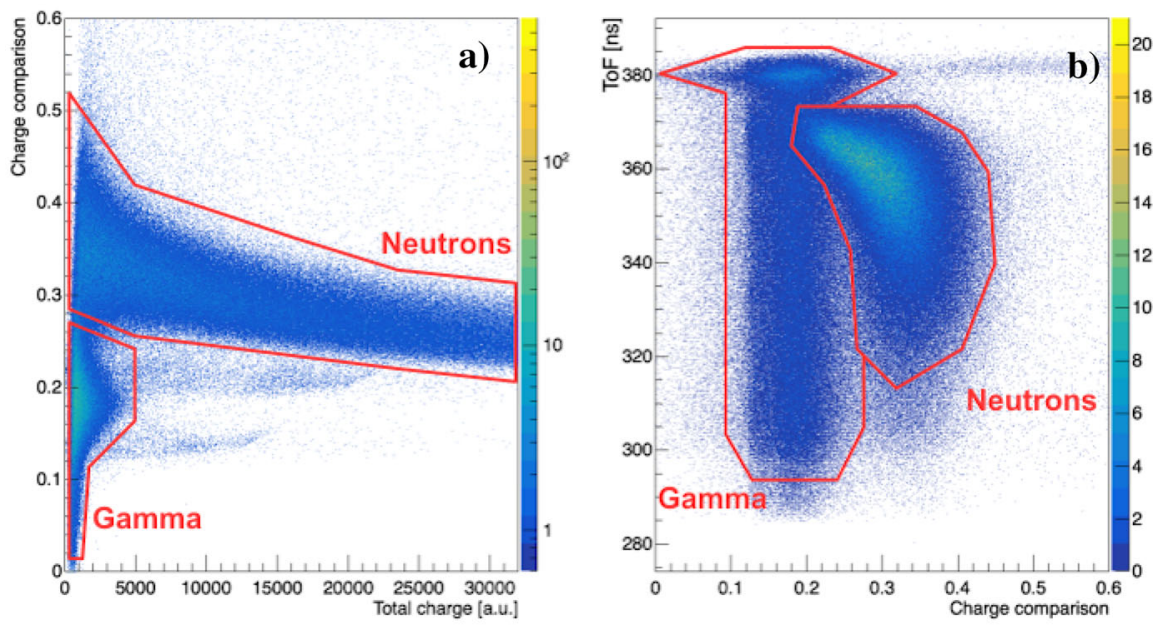

Fig. 14 GAMMA. Neutron identification in the NEDA array. a Corresponds to the charge comparison algorithm, while $\mathbf{b}$ shows the correlation between the time of flight obtained using the delayed RF of the beam as a stop and the individual signal as start and the $\mathrm{CC}$

or neutrons). In this context, inorganic scintillator materials are more suitable, since the performance in terms of energy resolution of the organic liquid scintillators, such as the EJ-301 used by NEDA (also due to the quenching effect), is quite limited. Moreover, the xylene-based liquid are quite difficult to manage in terms of safety and durability of the detectors since xylene is a powerful solvent. An inorganic scintillator is often constituted by an artificially grown crystal, with a volume that can vary from a few tenth of $\mathrm{mm}^{3}$ to a several $\mathrm{dm}^{3}$. The works of Refs. [190,191] discuss the performances of various inorganic scintillators, developed in recent years, with good neutron- $\gamma$ discrimination.

Modern materials such as the CLYC presents the advantage of combining a good energy resolution for $\gamma$-rays, slightly larger than the one of $\mathrm{LaBr}_{3}(4.5-5 \%$ at $661 \mathrm{keV})$ with a good neutron- $\gamma$ discrimination using PSA techniques. By applying a CC algorithm to the signals from the CLYC scintillator, one can obtain the separation of signals induced by $\gamma$ radiation and neutrons, as shown on panel b of Fig. 15 [192,193]. The red box highlights the contributions from thermal neutrons and $\gamma$-rays (depositing the same energy), which are located at PSA values around 0.8 and 0.7 , respectively (see [192] for more details on the identification mechanisms).

Scintillator materials are characterized by one or more scintillation-light decay constants. This feature uniquely defines the pulse line-shape; when the detector is a phoswich (i.e., a sandwich of scintillators), the different shapes associated with the various layers forming the telescope can be used to localize the material where the incident $\gamma$ radiation interacted. This is, for example, the case of the PARIS array, based on phoswich scintillation detectors [188] made of two crystals read by a single photomultiplier (PMT). Two types of phoswich are currently available: $\mathrm{LaBr}_{3}: \mathrm{Ce}-\mathrm{NaI}$ and $\mathrm{CeBr}_{3}-\mathrm{NaI}$. Both $\mathrm{LaBr}_{3}$ and $\mathrm{CeBr}_{3}$ are cubic $2^{\prime \prime} \times 2 " \times 2 "$ crystals while the $\mathrm{NaI}$ crystals are $2 " \times 2 " \times 6 "$. The two crystals are separated by a quartz window to avoid chemical 

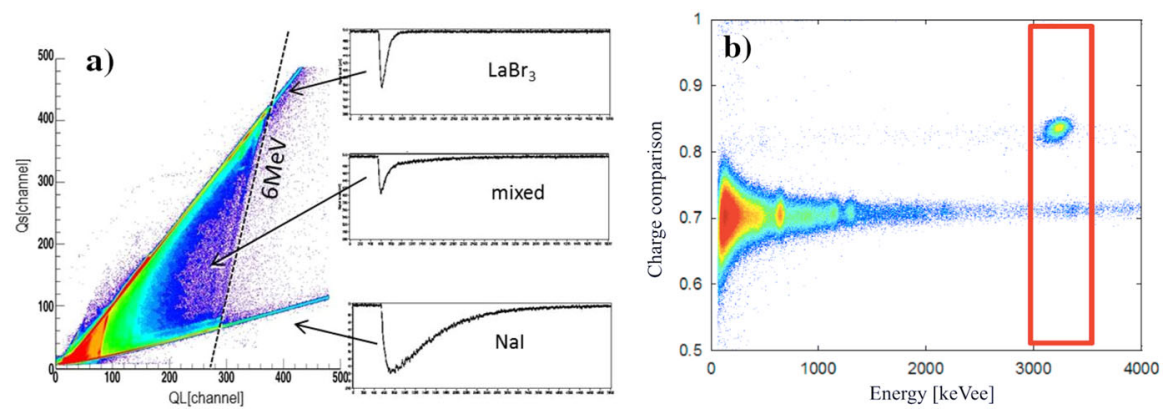

Fig. 15 GAMMA. a Correlation between the maximum amplitude $\left(\mathrm{Q}_{\mathrm{S}}\right)$ and the total integral $\left(\mathrm{Q}_{\mathrm{L}}\right)$ of the signal, plotted on the $y$ - and $\mathrm{x}$-axes, respectively, for a phoswich detector of the PARIS array. Adapted from Ref. [188]. b Neutron- $\gamma$ discrimination matrix obtained using a ${ }^{6} \mathrm{Li}$ enriched CLYC scintillator, with the charge comparison plotted against the total energy deposited. Figure adapted from [192]. The red box highlights the contributions from thermal neutrons and $\gamma$-rays (depositing the same energy), which are located at CC values around 0.8 and 0.7 , respectively [192]

interaction between them, that can lead in some years to an unwanted yellowing of the scintillators in proximity of the common edge. Although the two crystals have very different performances in terms of energy resolution $\left(\sim 4.1 \%\right.$ for the $\mathrm{LaBr}_{3}$ compared to $7.8 \%$ for the $\mathrm{NaI}$ at $662 \mathrm{keV}$ ), the difference in the time response of the two scintillators allows to identify in which crystal the interaction took place and get the best performances for both of them using a single PMT. In the present configuration, the PARIS array consists of up to 8 clusters made of 9 phoswich each. In Ref. [177], a PSA algorithm applied to $\mathrm{LaBr}_{3}: \mathrm{Ce}$ and $\mathrm{LaCl}_{3}: \mathrm{Ce}$ scintillators is discussed, to distinguish between signals induced by incident $\gamma$ rays and internal $\alpha$ activity. On panel a of Fig. 15 an example of charge comparison applied to PARIS detector is shown. Signals of the $\mathrm{LaBr}_{3}$ and $\mathrm{NaI}$ are given in inset of the figure. Since the $\mathrm{LaBr}_{3}$ signals are faster than the NaI, the short $\left(\mathrm{Q}_{S}\right)$ and long $\left(\mathrm{Q}_{\mathrm{L}}\right)$ integration window gives the same result, while in the case of $\mathrm{NaI}$ signal the short integration represents only a fraction of the longer one. This gives rise to two diagonal distributions: the steeper one represents the events in which the measured $\gamma$-ray interacted in the $\mathrm{LaBr}_{3}$ :Ce. The less steep diagonal is populated by $\gamma$-rays interacting in the NaI crystal. Mixed events are seen in between. The $6 \mathrm{MeV}$ line is referred to the $6.13 \mathrm{MeV} \gamma$-ray emitted from an excited state of ${ }^{16} \mathrm{O}$ produced by the reaction ${ }^{13} \mathrm{C}(\alpha, \mathrm{n}){ }^{16} \mathrm{O}$, taking place in the $\mathrm{Pu}^{13} \mathrm{C}$ radioactive source. Thanks to its large efficiency and good energy resolution, the PARIS array opens the way for $\gamma$-ray spectroscopy at medium to large $\gamma$-ray energy.

The signals produced by a scintillator have always the same line-shape, regardless of the radiation interaction point. As a consequence, the localization of the impact point requires the use of segmented photocathodes and the acquisition, on an eventby-event basis, of the image of the scintillation light on the photocathode [194-196]. This information is naturally provided by a SiPM array, while in the case of a PMT the use of a Position Sensitive PMT (PSPMT) is required.

The growth of the SiPM technology made a fine segmentation of the photocathodes possible. To better reconstruct the impact point, machine learning algorithms are under study [197]. Moreover, the research on ceramic scintillating materials will 
open new perspectives in terms of detector resolution, maximum available volume and cost/performance ratio.

\subsubsection{LUNA}

LUNA applies the PSA technique to discriminate the intrinsic background of the used detectors (due to their intrinsic radioactivity) with respect to the signal associated with neutrons. For the ${ }^{13} \mathrm{C}(\alpha, \mathrm{n}){ }^{16} \mathrm{O}$ measurement, a detector array based on $18{ }^{3} \mathrm{He}$ proportional counters at a pressure of 10 bars was designed. They are arranged in two concentric rings around the target chamber: 12 counters of $40 \mathrm{~cm}$ active length are located at a radius of $11 \mathrm{~cm}$, and 6 of $25 \mathrm{~cm}$ active length are located at $6 \mathrm{~cm}$ radius. Since the average neutron energy from the reaction is $2.5 \mathrm{MeV}$, counters are embedded in a polyethylene block used as moderator to obtain thermal neutrons for the final detection.

The output signal fed charge sensitive preamplifiers, whose signal was then digitized and directly written to disk for further analysis. Once the neutron is captured by ${ }^{3} \mathrm{He}$, the reaction ${ }^{3} \mathrm{He}(\mathrm{n}, \mathrm{p})^{3} \mathrm{H}$ takes place and two charged particles release their energy in the detector working as a proportional chamber.

Charge-sensitive preamplifier (i.e., current integrated) signals can be converted in current sensitive preamplifier signals by a first order digital high pass filter (CR). After the conversion, when a neutron is captured (and a p and ${ }^{3} \mathrm{H}$ are produced inside the detector) the output pulse has a typical structure with a double peak waveform (this is not true in few cases, i.e. in case of the wall effect $^{3}$ ) [198]. On the contrary, $\alpha$ background signals (due to the natural radioactivity of the Th and $\mathrm{U}$ chains coming from contaminants in the counter case) are characterized by only one particle releasing energy in the gas and the output signal has a single peak. In Fig. 16a, the reader can find an example of the filter performance: the blue and the dark green waveform are the preamplifier output and its CR shaping, respectively.

After the CR shaping, the PSA parameter $M$ can be defined as:

$$
M\left(t_{0}, t_{1}, t_{2}\right)=\frac{I\left(t_{0}, t_{1}\right)}{I\left(t_{0}, t_{1}\right)+I\left(t_{1}, t_{2}\right)},
$$

where $I(a, b)$ is the charge integrated between time $a$ and $b$. In the LUNA analysis $t_{1}-t_{0}=1 \mu \mathrm{s}$ and $t_{2}-t_{1}=7 \mu \mathrm{s}$ were used for the optimization of the neutron $/ \alpha$ PSD.

The method was tested plotting the M parameter vs energy deposited in a scatter plot for two sources: an AmBe source (almost only neutrons are detected by the setup) and a background measurement (mainly $\alpha$ particles are detected, because neutrons from outside were absorbed by borated PE). In Fig. 16b the main results are shown: red and blue points indicated events from AmBe source and environmental background, respectively. The acquisition time was about 1 day for the AmBe source and 1 week for the background, to acquire enough statistics for both dataset. After the test with these

\footnotetext{
3 i.e. when a neutron is captured near the counter surface and one of the two products cannot release all its energy inside the gas before exiting from the detector.
} 

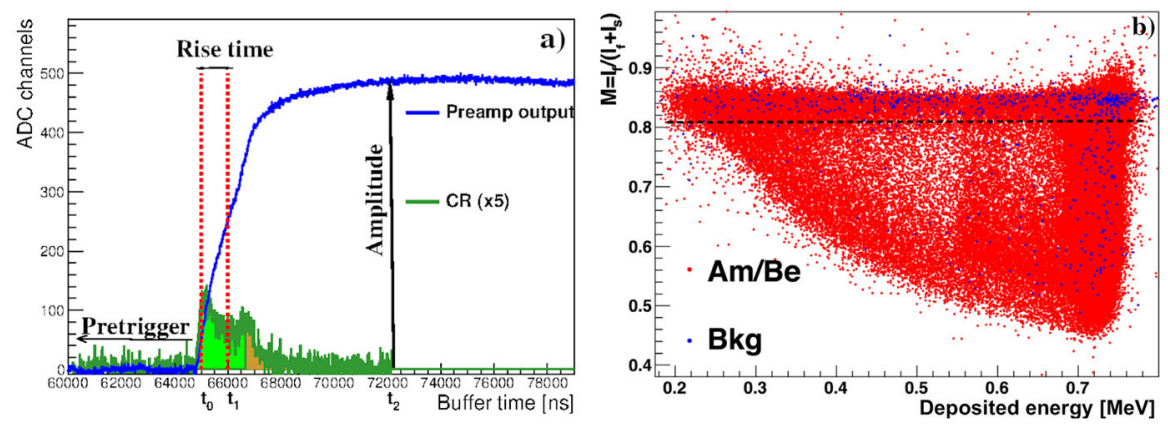

Fig. 16 LUNA. a The input charge sensitive preamplifier and filtered current sensitive preamplifier waveforms for a ${ }^{3} \mathrm{He}(\mathrm{n}, \mathrm{p})$ event. Typical features of the waveform, i.e. amplitude, rise-time and pre-sample are indicated by corresponding arrows. The signal refers to an event in which one neutron is detected. The light green and orange parts of the filtered waveform correspond to the fast and slow integral regions, respectively. Adapted from [116]. b Scatter plot of $M$ parameter-energy. Blue and red datasets indicate measurement of background and Am/Be source, respectively. From [116]

two data sets, the value for $M=0.81$ was chosen to maximize the neutron detection and the $\alpha$ rejection efficiencies, at about $90 \%$ and $98 \%$ of the level without PSA.

The use of these PSA techniques reduced the overall background of the detector from 3.3 down to 1 counts/h, allowing to measure lower cross sections, in the region of energies of astrophysical interest [115]. With the installation of the facility LUNA MV at LNGS foreseen in the end of this year, the LUNA scientific program will include the ${ }^{22} \mathrm{Ne}(\alpha, \mathrm{n})^{25} \mathrm{Mg}$ cross section measurement [199]. The described PSA method will allow also in this case to increase the sensitivity of the measurement providing for the first time direct cross section data below the $704 \mathrm{keV}$ resonance.

\subsection{4 n_TOF}

In $n \_$TOF, the PSA technique is used to discriminate among $\gamma, \alpha$ particles and neutrons. The detection of the prompt $\gamma$ rays resulting from the neutron capture events, i.e. the electromagnetic transitions leading to the de-excitation of the compound nucleus, is performed using either a calorimeter made of $\mathrm{BaF}_{2}$ crystals [200] (called Total Absorption Calorimeter) or an array of $\mathrm{C}_{6} \mathrm{D}_{6}$ liquid scintillators [201]. The calorimeter is designed to detect the full $\gamma$-ray cascade using detectors of high intrinsic efficiency and large solid-angle coverage [200]. The other experimental setup is based on a detector with extremely low neutron sensitivity and exploiting the total energy technique [202] in combination with the pulse height weighting technique.

The main background source is related to the neutrons scattered by the sample and interacting with the surrounding materials. This background component is particularly critical as it follows the same energy dependence and resonant pattern as the capture events.

The different operating principles of the two detectors require a good $\alpha / \gamma$ and $\mathrm{n} / \gamma$ discrimination. A convenient solution to perform particle identification and, in the mean time, to identify and reconstruct pile-up events generated by the high instantaneous neutron flux is based on the acquisition of the complete waveforms of the 

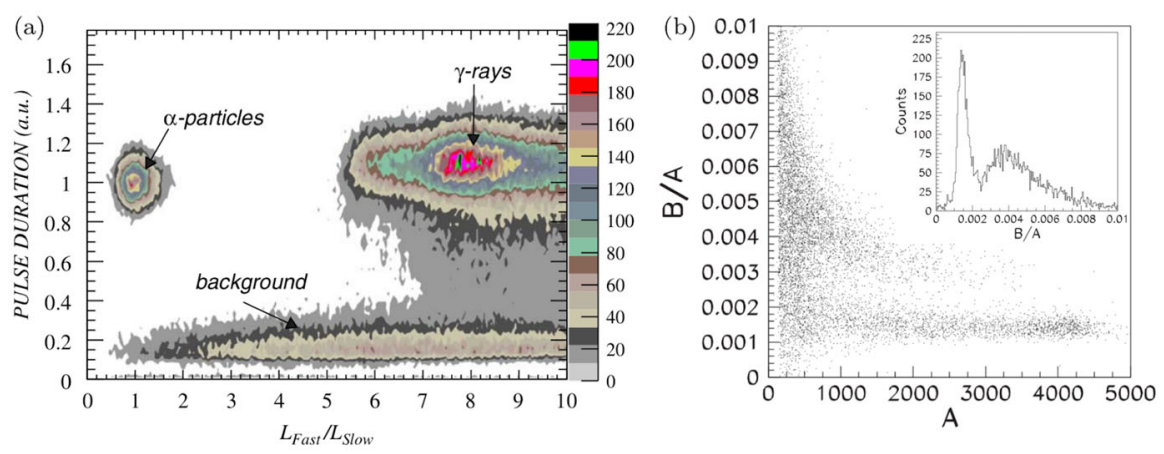

Fig. 17 n_TOF. a TAC detector: Signal duration versus the ratio of the fast and slow component of the scintillation light output. From [205]. b C $_{6} \mathrm{D}_{6}$ liquid scintillators: ratio between the slow and fast component of the light response as a function of the fast component. The upper and lower branches correspond to neutrons and $\gamma$, respectively (projection of $B / A$ ratio shown in the inset; the first peak is associated with $\gamma$ and the second peak corresponds to neutrons). Adapted from [207]

signals, using Flash Analogue to Digital Converters (FADC). The important advantage of this approach is the possibility of deducing the physical information contained in the digitized signals through off-line analyses.

Total Absorption Calorimeter (TAC) This is a segmented $4 \pi$ detector array made of $40 \mathrm{BaF}_{2}$ crystals suited for neutron capture cross-section measurements on low-mass and/or radioactive samples. The light output of the $\mathrm{BaF}_{2}$ has two main components with decay times of $(0.6-0.8 \mathrm{e}) \mathrm{ns}$ and $630 \mathrm{~ns}$ for the fast and the slow emission, respectively. The crystals are 12 pentagonal and 28 hexagonal in shape, and each of them is coupled with a $12.7 \mathrm{~cm}$ Photonis XP4508B PMT.

The neutron sensitivity is minimized thanks to a spherical neutron moderator/absorber made of an inert non-flammable lithium salt $\left(\mathrm{C}_{12} \mathrm{H}_{20} \mathrm{O}_{4}\left({ }^{6} \mathrm{Li}\right)_{2}\right)$ inserted in the central cavity, surrounding the sample, and also by the encapsulation of each crystal in a ${ }^{10} \mathrm{~B}$-loaded carbon-fiber shield. The neutrons can be captured by the absorber producing a $\gamma$ cascade, which can be recognized by the Total Absorption technique, i.e. reconstructing the deposited-energy distribution of the corresponding $\gamma$ cascades [203].

The other non-negligible background contribution is due to the radioactive impurities in the $\mathrm{BaF}_{2}\left({ }^{226,228} \mathrm{Ra}\right.$ and their daughters), which emit $\alpha$ and $\gamma$-rays with energies up to a few MeV [204]. Signals produced by $\alpha$-particles can be discriminated from $\gamma$-induced events by the absence of the fast component in the light output; the time dependence of each light component can be expressed by an exponential formula with a proper decay constant. Therefore, the $\alpha / \gamma$ discrimination is achieved by fitting the pulse shape. The event selection is based on the total pulse duration versus the ratio of the fast over the slow components of the scintillation light output (Fig. 17a).

In addition to rejecting background events, identification of $\alpha$ particles permits one to continuously monitor the gain of each module by the position of the energy spectrum lines of radium and its progeny [204]. Moreover, the information on the multiplicity (i.e. the number of hits registered in $\mathrm{BaF}_{2}$ crystals) can be used for the 
selection of $\gamma$-ray events, as the range of charged particles is much smaller than the crystal dimension.

$C_{6} D_{6}$ liquid scintillators $\mathrm{In}$ the array of $\mathrm{C}_{6} \mathrm{D}_{6}$ liquid scintillators each module consists of a cylindrical cell with carbon-fiber walls, $12 \mathrm{~cm}$ in diameter and $9 \mathrm{~cm}$ thick, read out by an EMI 9823QKA PMT [206]. The low sensitivity to background signals induced by scattered neutrons is achieved by the minimization of the amount of material surrounding the detector and using only materials characterized by a small neutron capture cross section. However, since the component of neutron diffusion is particularly significant, it is necessary to take it carefully into account. In fact the neutron scattering cross section can be some order of magnitude larger than capture $\left(\sigma_{\mathrm{n}} / \sigma_{\gamma} \sim 10^{3}-10^{4}\right)$. Also in this case the discrimination $\mathrm{n} / \gamma$ is performed through the shape of the signal produced by the two particles in the detector. The signal shape analysis from scintillation detectors can be obtained by the convolution of the exponential decay spectrum of the scintillation light with the response function of the PMT and readout system [207]. Since the decay constants of the scintillator do not depend on the particle type, the only expected difference between $\gamma$ - and neutron-induced signals is due to the relative contributions of the fast and slow components. The branches corresponding to neutrons and $\gamma$ are well identified in the slow to fast component ratio as a function of the fast component (Fig. 17b).

\subsubsection{NUCLEX}

The detection of fast neutrons with respect to the $\gamma$ background can be achieved with commercially available solid scintillators.

In organic systems, the scintillation process is based on the energy transfer between aromatic groups of the either solid or liquid matrix and the dispersed dye molecules characterized by high quantum efficiency. In fast neutrons and $\gamma$ particles detection, the particles actually measured by the scintillators are recoil protons from fast neutrons and recoil Compton electrons from $\gamma$ rays. Protons produce, along their path, a higher ionization density (which causes the emission of delayed photons) with respect to electrons. This delayed fluorescence has characteristic times of the order of tens of $\mathrm{ns}$, with respect to the prompt emission whose time scale is 1-2 ns, maintaining the same spectral response. Therefore recoil protons generate scintillation pulses with a more intense long living component with respect to Compton electrons, thus allowing their discrimination through pulse shape identification techniques, as shown in Fig. 18, where the $n / \gamma$ discrimination obtained with solid polyoxane-based scintillators (cylinder shaped with a 2" diameter and $2 \mathrm{~cm}$ height, doped with $8 \%$ PPO) is shown. The scintillator was irradiated with a pulsed flux of $4.5 \mathrm{MeV}$ neutrons from $\mathrm{p}+{ }^{7} \mathrm{Li}$ reactions.

This research line is in progress with siloxane-based scintillators highly doped (up to $8 \%$ in weight) with PPO (2,5-diphenyloxazole) [208]. 


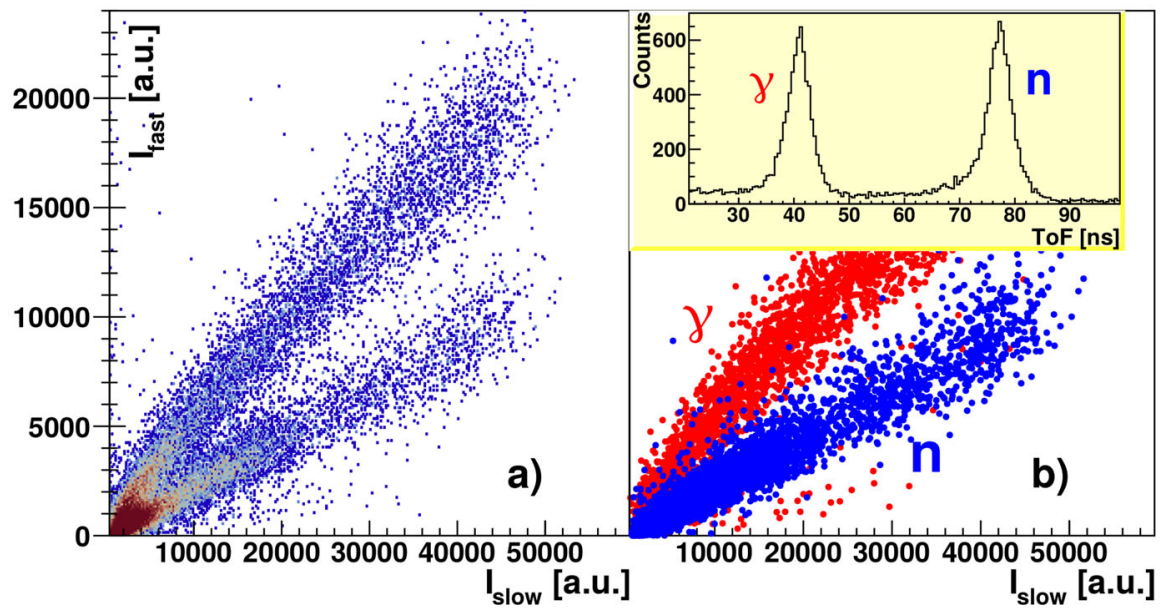

Fig. 18 NUCLEX. $\mathrm{n} / \gamma$ discrimination with solid polysiloxane-based scintillators. a PSD correlation plot between fast and slow components obtained through shapers with different integrating constant. b The same correlation where colours indicate particle identification obtained with an independent method (i.e. time of flight measurement, as shown in the inset)

\section{4 $\gamma$-ray tracking with HPGe detectors}

The PSA can be used to perform the $\gamma$-ray tracking, to significantly improve the quality of the $\gamma$ spectroscopy which can be achieved with an HPGe array. One of the most important positive effects of this technique is the correction of the Doppler effect.

\subsubsection{GAMMA}

The AGATA array [60,210,211], designed and built by an international collaboration in which GAMMA significantly contributes, fully exploits the PSA technique to obtain extremely good position and energy resolutions. The key element required to perform the $\gamma$-ray tracking is to build a position sensitive HPGe crystal. This can be achieved using detector segmentation in conjunction with the possibility to sample and process the output signals from the detector pre-amplifiers. The detector segmentation is realized, for obvious production limits, on the outer surface of the germanium crystal. A peculiar geometry of dopants, conduction overlays and isolation trenches divide the AGATA crystals surface into 36 areas, called "segments" [210]. The inner part of the detector presents a highly doped cylindrical cavity, that constitutes the inner common detector electrode connected to the high voltage. The outer surface is n-doped, while the inner one is p-doped and collects electrons. For this reason the energy information is generally taken from the common electrode: in fact, the neutron damage produces amorphous pockets that generally work as hole traps that leave the electron signal mostly unaffected.

Different interaction positions inside the crystal lead to the release of free charge carriers with different spatial distributions. The drift motion of these carriers across the detector volume following the electric field lines induces current signals not only at 
the terminals of the segment where the interaction happened (signal that contains the energy information and the radial position) but also on the nearby segments, according to the Ramo theorem. Since the preamplifiers bandwidth is high enough to preserve the detector signal high-frequency content, it is possible to retrieve the information about the interaction position inside the crystal analyzing the leading edge of the preamplifier wave-forms.

To keep the position information, a dedicated differential preamplifier was developed [212]. Signals coming from the 36 segments and the central contact are continuously digitized using high-resolution, 100 Msps (Megasample per second) ADC. A specific readout electronics and trigger have been developed, allowing AGATA to maintain optimal performances, in terms of energy resolution and deadtime with rates up to $80 \mathrm{kHz}$ per crystal.

To reconstruct the position information, each AGATA crystal must be previously characterized. Illuminating the detector with precisely collimated source with known position (with the support of GEANT4 simulations, see Ref. [213]) it is possible to build a digital library of preamplifier signals. Incoming signals are then compared to the reference ones using grid search algorithms. The analysis is performed online, with dead-time below few percents, up to rates of about 4-5 kHz per crystal.

Typically, the PSA will give the interaction positions within the crystal with a resolution of the order of $5 \mathrm{~mm}$ FWHM [214]. Implementation of position uncertainty determination is currently under study with, for example, bootstrapping methods [215].

The first main improvement due to the PSA is related to the fact that thanks to the position determination inside the crystals, the interaction path of a $\gamma$ ray inside the array can be reconstructed, allowing for the detection of its full energy. Several algorithms have been developed over the years and are still being improved [216]. Reconstruction of the full energy of the $\gamma$ rays, taking into account all the possible interactions processes: photo-electric, Compton-scattering, pair-creation at the typical energies observed in nuclear structure studies (few $\mathrm{keV}$ to hundreds of $\mathrm{MeV}$ ) allows to reach performances in terms of $\mathrm{P} / \mathrm{T}^{4}$ close to the one of Compton-Suppressed arrays, with a huge efficiency gain.

Second improvement, thanks to the precise determination of the $\gamma$-ray interaction point in the crystal, the probability of the charge carriers to be trapped at local defects can be evaluated, allowing the correction of the measured energy.

The third main result of the position resolution, obtained with the PSA, is the unprecedented Doppler correction capabilities, when AGATA is coupled to spectrometers allowing for the measurement of the velocity or to high-resolution charge particle detectors and the kinematic reconstructions of the recoil vector is performed. In this case, it is possible to obtain energy resolution only $\sim 5 \%$ larger than what is observed for $\gamma$ rays emitted at rest, thus allowing to obtain unprecedented results for the study of exotic nuclei and also opening new perspectives in the study of high-energy $\gamma$ rays [209].

In Fig. 19a, the energy resolution which can be obtained by AGATA fully exploiting the PSA is shown as a red curve, to be compared with the grey line obtained without

\footnotetext{
4 peak-to-total ratio, meaning the ratio between the events in the photo-peak and the one in the Comptonscattering region.
} 

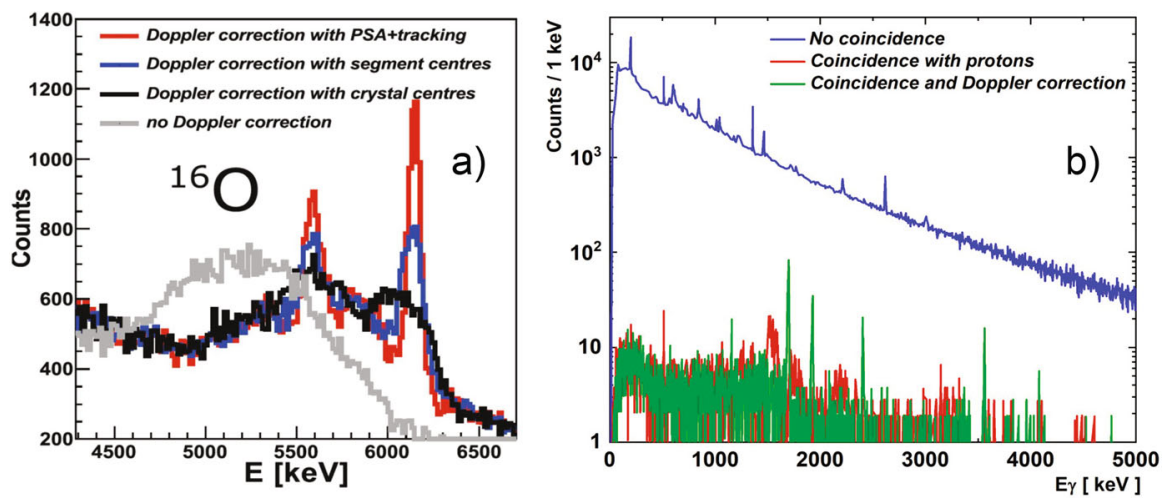

Fig. 19 GAMMA. a Energy spectrum measured by AGATA in the region of the $6.13 \mathrm{MeV}$ gamma-ray, emitted by the ${ }^{16} \mathrm{O}$ nucleus with a recoil velocity $v / c=20 \%$. The grey spectrum is without Doppler correction, while the others were corrected using different position information, as described in the legend. From [209]. b Performances of AGATA during a radioactive ion-beam experiment. The SPIRAL1 postaccelerated ${ }^{19} \mathrm{O}$ beam was impinging on a $3-\mu \mathrm{m}$-thick $\mathrm{CD}_{2}$ target deposited on a $11 \mu \mathrm{m}$ Au backing for lifetime measurement in ${ }^{20} \mathrm{O}$. In the inset the comparison between the Doppler corrected and non corrected spectra in linear scale is shown. The presence of the high energy transitions, above $2500 \mathrm{keV}$, can only be observed thanks to the unique performances of the setup

Doppler correction, as explained in the caption. In Fig. 19b, one of the latest results of the AGATA array, using a post-accelerated radioactive ion beam at GANIL, is reported. A $10^{5}$ pps ${ }^{19} \mathrm{O}$ beam was produced and post-accelerated to $8 \mathrm{MeV} /$ nucleon at SPIRAL1 (GANIL, France); it impinged on a deuterated polyethylene $\left(\mathrm{CD}_{2}\right)$ target deposited on a thick $\left(20 \mathrm{mg} / \mathrm{cm}^{2}\right)$ Au backing. The direct spectrum (blue curve) is dominated by the background radioactivity (natural radioactivity and radioactive beam implantation). Combining the detection of a proton and the detection of the recoiling nucleus with the position of the first interaction of the $\gamma$ ray within AGATA, it is possible to obtain the green line, which corresponds to the Doppler corrected spectrum of ${ }^{20} \mathrm{O}$ populated by the ${ }^{19} \mathrm{O}(\mathrm{d}, \mathrm{p})$ transfer reaction, with an energy resolution similar to that obtained at rest. Therefore the line shape of the transition can be used to perform direct lifetime measurement even with relatively low statistics ( $\sim 200$ counts) in the photopeak.

In the next future, the solid angle coverage of the AGATA array will be progressively increased from the current $1 \pi$ to $2 \pi$, thus requiring an increase of high-resolution readout channels up to 1100; new back-end electronics is currently under test. Moreover, new algorithms for the PSA and $\gamma$-ray tracking based on machine learning algorithms are under study.

\section{$5 \mathrm{X}$ and $\gamma$ spectroscopy}

In many nuclear physics experiments, the reaction products cannot be directly detected and identified. This may be caused either by a short lifetime of the searched products or by their low energy, not allowing them to leave the target or to be directly measured. However, since these products have internal structures, they emit characteristic X or $\gamma$ 
radiation that can be used to tag their presence in the reaction. The large mean free path of $\mathrm{X}$ and $\gamma$ rays allows to detect them outside of target or interaction region and their identification requires a good spectroscopic capability, to resolve interesting energy lines from, generally large, correlated and uncorrelated backgrounds. The uncorrelated background, typically, can be reduced by a narrow-gate coincidence with other detectors, suggesting to select fast spectrometers.

The spectroscopy of X-rays is typically performed with silicon drift detector (SDD) combining high-energy resolution of about $100 \mathrm{eV}$ with very fast response time of the order of $1 \mathrm{~ns}$. Also charge-coupled device (CCD) can be used when background rate is not overwhelming.

For $\gamma$-ray spectroscopy HPGe detectors with their resolution of few $\mathrm{keV}$, high efficiency and fast response are most indicated for this kind of experiments. Inorganic scintillator crystals are less expensive and much easier to handle, providing similar efficiency and timing, but with 20-60 times larger energy resolution.

In this section, we describe X-ray spectroscopy by SDD and CCD and $\gamma$-ray spectroscopy with HPGe and CsI(Tl) detectors as used in KAONNIS, PANDORA and CHIRONE experiments.

\subsection{CHIRONE: identification of Pygmy Dipole Resonance and of ${ }^{12} \mathrm{C}$ excited states with $\gamma$ spectroscopy}

The CHIMERA $4 \pi$ multidetector at LNS is primarily a charged particle detector. However, the $\mathrm{CsI}(\mathrm{Tl})$ crystals, belonging to the spherical part and read by a photodiode [217] (see Sect. 3.2.1), are also suitable to detect $\gamma$-rays due to the high atomic number of the material. Indeed, due to the large solid angle coverage of the single detector and to the small yield of charged particles, the sphere has a larger signalto-noise ratio for $\gamma$-detection with respect to the forward part of CHIMERA. The efficiency of the crystals depends both on $\gamma$ energy and thickness of CsI(Tl); as an example an efficiency of $\approx 50 \%$ is obtained in the energy range of $4-5 \mathrm{MeV}$. The energy resolution is $500 \mathrm{keV}$, while timing resolution is $\approx 100 \mathrm{~ns}$. Such CHIMERA peculiarities allow to detect $\gamma$-rays in a wide energy range (from an energy threshold of $\approx 1 \mathrm{MeV}$ to at least $20 \mathrm{MeV}$ ), with a good detection efficiency, and to reconstruct their angular distributions. The method used for the identification of $\gamma$-rays is based on the so-called fast-slow technique described in Sect. 4.1.

With this method, the collaboration has performed several experiments in the framework of the $\gamma$-ray spectroscopy. The energy resolution of CsI(Tl) can not compete with HPGe detectors, however the large solid angle and the efficiency allow to extract data in cases of low-intensity radioactive beams or for broad resonances, as, for instance, the Pygmy Resonance. Two examples are described in the following.

The collaboration has investigated the $\gamma$-decay of ${ }^{12} \mathrm{C}$ levels, in particular the Hoyle and the $9.64 \mathrm{MeV}$ states [218]. In this case, the main advantage of $\mathrm{CsI}(\mathrm{Tl})$ crystals is the high efficiency that allows to access information about the levels with a low decay probability. Figure 20a shows $\gamma$-ray energy spectra, measured in the reaction $\alpha+{ }^{12} \mathrm{C}$. In the figure one might note that it was possible to observe the $12.7 \mathrm{MeV}$ $\gamma$-decay of ${ }^{12} \mathrm{C}$ level with a relative probability of occurrence of $4 \times 10^{-5}$, obtained 

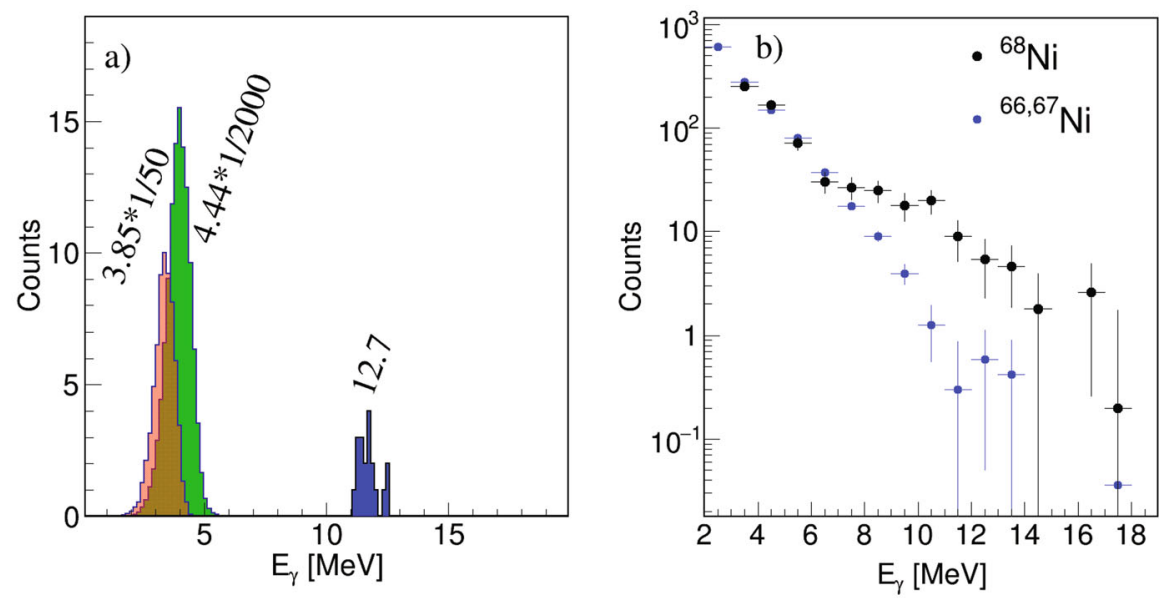

Fig. $20 \mathrm{CHIRONE}$. a $\gamma$-ray energy spectra measured with CHIMERA $\mathrm{CsI}(\mathrm{Tl})$ in the reaction $\alpha+{ }^{12} \mathrm{C}$ at $16 \mathrm{MeV} /$ nucleon (figure adapted from Ref. [219]). Various levels of $\mathrm{C}$ are indicated with different colours and their scale factors. $\mathbf{b} \gamma$-ray energy spectra in ${ }^{68} \mathrm{Ni}+{ }^{12} \mathrm{C}$ reaction, Doppler shift corrected. The black dots represent the spectrum in coincidence with ${ }^{68} \mathrm{Ni}$, detected at forward angles. The blue dots represent the spectrum in coincidence with ${ }^{66,67} \mathrm{Ni}$. The bump at around $10 \mathrm{MeV}$ is the contribution of the Pygmy Dipole Resonance decay (figure adapted from Ref. [144])

as a combination of cross section and $\gamma$-decay probability of the channel. Kinematic methods are also useful to improve resolution and background suppression (see Sect. 9.1.2).

Another relevant topic concerns the investigation of the Pygmy Dipole Resonance in the exotic nucleus ${ }^{68} \mathrm{Ni}$ above the neutron emission threshold [144]. Figure 20b shows $\gamma$-ray energy spectra obtained in the reaction ${ }^{68} \mathrm{Ni}+{ }^{12} \mathrm{C}$ at $28 \mathrm{MeV} /$ nucleon. The enhancement at around $10 \mathrm{MeV}$ comes from the contribution of the Pygmy Dipole Resonance decay of ${ }^{68} \mathrm{Ni}$. The cross section of such Pygmy decay was evaluated from the difference between spectra measured in coincidence with ${ }^{68} \mathrm{Ni}$ and ${ }^{66,67} \mathrm{Ni}$, as discussed in detail in Ref. [144].

The collaboration is currently improving the detection of $\gamma$-rays, employing the new electronics [187], that allows the digitization of the total shape of signals. The offline computation on the pulse waveform, using several filters, will allow to improve the identification of $\gamma$-rays, based on the fast-slow method, and to remove, in a better way, the background.

\subsection{KAONNIS: kaonic atom identification by X-ray spectroscopy}

In SIDDHARTA (see Sect. 2.1.3), large-area X-ray detectors with spectroscopic capabilities were required to measure the low yield of X-rays from kaonic atom transitions, isotropically emitted from the large-volume gaseous targets used to enhance the $\mathrm{K}^{-}$ stopping. The silicon-based solid-state devices have typical values of FWHM $<160 \mathrm{eV}$ at $\mathrm{Fe}_{\mathrm{K}}(6.4 \mathrm{keV}), \mathrm{a} \simeq 90 \%$ detection efficiency in the $5-10 \mathrm{keV}$ range and a linearity of 

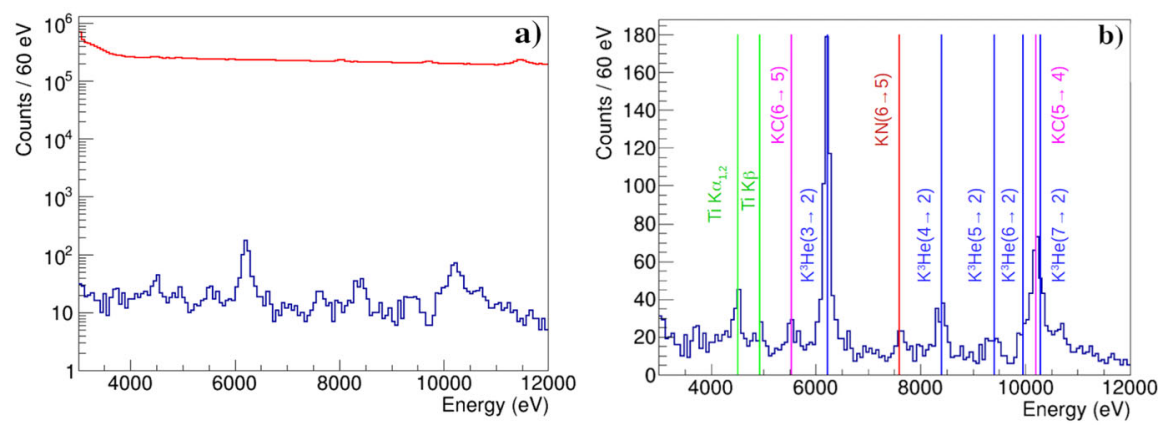

Fig. 21 KAONNIS. a Effect of the triple coincidence implemented in the SIDDHARTA experiment [3235]. The spectra of the SDDs corresponding to $9 \mathrm{pb}^{-1}$ of integrated luminosity for $\mathrm{K}^{3} \mathrm{He}$ run with (blue) and without (red) the triple coincidence between TOF and SDDs. b Background subtracted $\mathrm{K}^{3} \mathrm{He}$ spectrum. The green lines identify the titanium $\mathrm{K}_{\alpha, \beta}$ peaks, coming from the calibration targets, the red and pink ones identify, respectively, signals from the kaonic Carbon and Nitrogen formed in the Kapton target cell, while the blue ones correspond to the 7, 6, 5, 4, 3 $\rightarrow 2 \mathrm{p} \mathrm{K}^{3} \mathrm{He}$ transitions

$\Delta E / E<10^{-3}$ : for these properties, SDD were selected as the best option in terms of achievable spectral resolution.

In addition, the requirement of few hundreds of ns read out times, to exploit the possibility to trigger the acquisition of the X-ray signals and reduce the machine background, oriented the final choice on $1 \mathrm{~cm}^{2}$ SDD [28,29]. In the SIDDHARTA experiment, indeed, a triple timing coincidence between the TOF system and SDDs (see Sect. 6.1.2) allowed to reduce the background dramatically, as shown in Fig. 21. In panel a, the comparison of SDD spectra with and without the triple coincidence show an overall background reduction by a factor $\simeq 10^{5}$. In panel $\mathrm{b}$, the lines of interest are the blue ones, corresponding to the $\mathrm{K}^{3} \mathrm{He}$ transitions to the $n=2$ level; the $\mathrm{K}_{\alpha}$ line, corresponding to the $3 d \rightarrow 2 p$ transition is clearly visible, as well as the $4 \rightarrow 2$. Like for kaonic hydrogen, the interesting parameters to be extracted are the strong interactioninduced shift and width of the atomic levels. The very good resolution of the SDD allows to extract the almost $-300 /+600 \mathrm{eV}$ shift/width of the kaonic hydrogen $n=1$ level [32,33] and $-800 /+750 \mathrm{eV}$ of kaonic deuterium [36]; unfortunately it is not possible to properly measure the width of the kaonic helium $n=2$, which are expected to be at the level of eV [34,35].

Therefore, the energy resolution of SDDs is limiting kaonic atom X-ray spectroscopy. Bragg spectrometers, owning a much higher resolution, were not used so far because of their extremely low efficiencies and very small source size. In the future, measurements with sub-eV precision could be achieved, thanks to the development of the Highly Annealed Pyrolytic Graphite (HAPG) mosaic crystals. The VOXES collaboration at LNF [220-222] reported the possibility to combine HAPG crystal properties with the vertical focus of the Von Hamos configuration, to realize a spectrometer able to maintain a resolution in the order of $0.1 \%(\mathrm{FWHM} / E)$, for energies below $10 \mathrm{keV}$, and of $0.3 \%$ up to $20 \mathrm{keV}$, using a source size ranging from $500 \mu \mathrm{m}$ to $2 \mathrm{~mm}$ in the Bragg dispersion plane. This development paves the way for deeper understanding of the low energy strangeness QCD. 

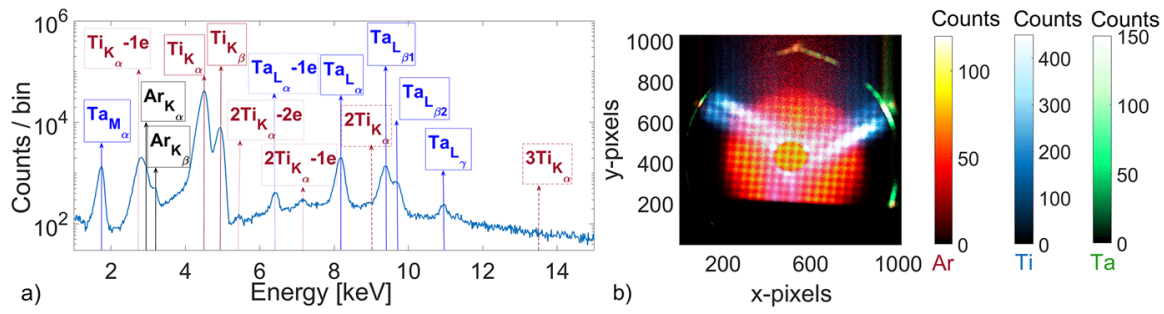

Fig. 22 PANDORA. a X-ray spectrum collected with the pin-hole CCD system in SPhC mode. b SPhC image of fluorescence X-rays coming from plasma (Ar, red colour-scale) and from plasma chamber walls materials ( $\mathrm{Ti}$ and $\mathrm{Ta}$, blue and green colour-scale, respectively). Figure adapted from Ref. [227]

\subsection{PANDORA: ion identification with X-ray spectroscopy and isotope identification with $\gamma$ spectroscopy}

The most relevant goal of the PANDORA project is to correlate the nuclear decay rate to the plasma environment properties, by simultaneously measuring $\mathrm{X}$ and $\gamma$ rays. This can be achieved through an innovative multi-diagnostic detection system based on a simultaneous identification and discrimination of the photons emitted by the plasma (from microwave to hard X-ray) and the $\gamma$-rays emitted after the isotope $\beta$-decay.

The overall structure of PANDORA experimental setup consists of three main pillars (see also Sect. 2.4.5): Magnetic Trap, Array of 14 HPGe detectors and Plasma multi-diagnostics system [122,223].

Due to the inhomogeneous and anisotropic nature of magnetoplasma the identification of plasma self-emitted photons has to be done using space and time-resolved techniques. Quantitative elemental composition and plasma morphology investigations are performed by the powerful pin-hole CCD technique, in single photon-counted mode (SPhC) $[224,225]$. It allows to perform high-resolution soft X-ray space-resolved spectroscopy, thus evidencing the local displacement of electrons at different energies, as well as of plasma ions, highlighted by fluorescence line emission. The setup consists of a CCD camera (sensitive range $2-20 \mathrm{keV}, 1024 \times 1024$ pixels), a $\mathrm{Pb}$ pin-hole ( $400 \mu \mathrm{m}$ of hole diameter) and a $\mathrm{Pb}$ multi-disk collimator designed to increase signalto-noise ratio [226]. In SPhC mode, thousands of image-frames are acquired by setting an exposure time to tens of $\mathrm{ms}$ and by extracting a spectrally resolved image and a space-resolved spectrum. The system reaches high spatial and energy resolutions of $500 \mu \mathrm{m}$ and $0.326 \mathrm{keV}$ at $8.1 \mathrm{keV}$, respectively. Figure 22a shows a typical X-ray SPhC spectrum, where it is possible to identify X-rays coming from plasma due to ionized $\mathrm{K}_{\alpha}$ Ar lines and X-rays emitted from materials of the plasma chamber walls (due to excited Ti and Ta). As shown in Fig. 22b, using the same $\mathrm{SPhC}$ image is it also possible to distinguish the spatial emission coming from Ar plasma, compared to the Ti axial losses or the Ta radial losses. SPhC image analysis allows to measure plasma radius with an uncertainty of about 5\%. In perspective, using two CCD pin-hole cameras, it will be possible to estimate also the plasma volume.

The plasma environment properties can thus be correlated to nuclear $\beta$-decay, determined through $\gamma$-ray spectroscopy. The design of $\gamma$-ray spectroscopy system was 
optimized by GEANT4 simulations. Since the harsh environment (the noise is represented by the intense plasma self-emission) strongly affects the signal-to-noise ratio, HPGe ( $70 \%$ of relative efficiency) detectors were chosen for their high resolution $(0.2 \%$ at $1 \mathrm{MeV})$ [228]. Due to limited number of possible holes in the magnetic trap, the best compromise was obtained using 14 HPGe detectors. The total photopeak efficiency of the array, including the geometrical acceptance, was estimated between 0.1 and $0.2 \%$, depending on the energy of the detected $\gamma$-ray. With this setup the in-plasma (expected) lifetime measurements for the main targets of this experiment are expected to take from a couple of weeks for the simplest physical case $\left({ }^{134} \mathrm{Cs}\right)$ to about 3 months for the long living ${ }^{176} \mathrm{Lu}$ case.

\section{Particle identification by means of time of flight combined with energy or magnetic field analysis}

In this section, PID techniques based on TOF measurements, associated with magnetic analysis or energy measurements, are described. In the first case measurements of the start/arrival time and track length provide the velocity, while the momentum is measured via a magnetic spectrometer. In the second case, usually adopted for lowmomentum nuclei, the identification is performed correlating timing measurement with the energy loss inside the detector or the energy measured for completely absorbed nuclei: while the former provides $Z$ discrimination, the latter is sensitive to mass, but sometimes the solution adopted profits of both approaches.

In this section, the experiments are grouped on the basis of the momentum range of the identified particle or nuclei, above or below $\approx 100 \mathrm{MeV} /$ nucleon. In the first group the experiments are mainly interested in single particle or very light nuclei identification, while in the second one the studies concentrate on nuclei production. The time resolution requirements depend on the type and momentum of the analysed particle/nuclei, ranging from tens of ps up to few ns. A variety of detectors are used, including gas detectors like Multigap Resistive Plate Chambers (MRPC) or MicroChannel Plates (MCP), scintillating materials or silicon sensors.

\subsection{Particle and very light nuclei identification in the $\approx 100 \mathrm{MeV}$ to $\mathrm{GeV}$ energy range}

\subsubsection{ALICE TOF: MRPCs for particles up to $5 \mathrm{GeV} / \mathrm{c}$}

The time of flight detector [229-231] is one of the ALICE central barrel detectors and it is based on the Multigap Resistive Plate Chambers (MRPC) technology with ten gaps of $250 \mu \mathrm{m}$. It is located at $3.7 \mathrm{~m}$ from the beam axis and it covers a cylindrical area of $141 \mathrm{~m}^{2}$ at $-0.9<\eta<0.9$ with a total of 153,000 readout pads of area $3.5 \times 2.5 \mathrm{~cm}^{2}$. A time resolution of less than $50 \mathrm{ps}$ has been measured on a test beam for the single MRPC strip [232]. The ALICE TOF can both provide charged particle PID in the intermediate momentum range and a trigger for cosmic ray events and 

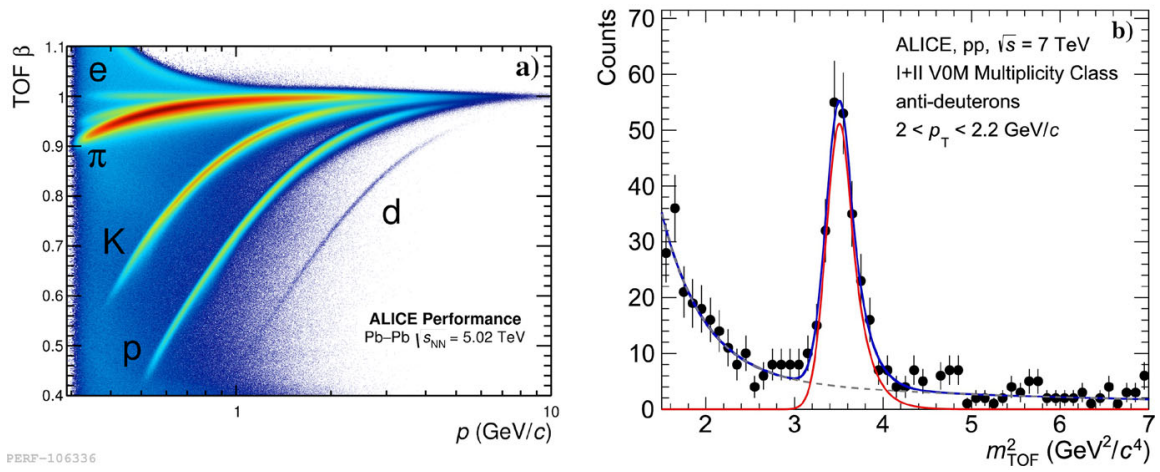

Fig. 23 ALICE. a Particle velocity $(\beta=\mathrm{v} / c)$ measured by the TOF as a function of momentum in $\mathrm{Pb}-\mathrm{Pb}$ collisions at $\sqrt{s_{\mathrm{NN}}}=5.02 \mathrm{TeV}$ (from [233]). Tracks with transverse momentum lower than $0.3 \mathrm{GeV} / c \mathrm{do}$ not reach the TOF due to the magnetic field. b Squared-mass distribution around the anti-deuterium mass as calculated by TOF starting from a selection in the energy loss in the ALICE TPC (from [235])

ultraperipheral collisions. Since its installation in 2008, no sign of degradation has been observed [233].

A simultaneous momentum and time-of-flight measurement can lead to the mass determination. The momentum is provided by the tracking of the ALICE TPC and ITS detectors placed inside the 0.5 T ALICE magnet (see Sect. 3.3). Two particles with the same momentum but different masses, $i$ and $j$, after travelling a distance $L$ will have a time difference of

$$
\Delta t_{i j} \simeq \frac{L c}{2 p^{2}}\left(m_{i}^{2}-m_{j}^{2}\right) .
$$

The final PID with the TOF detector can be performed considering the difference $\Delta t=t_{\mathrm{TOF}}-t_{\text {event }}-t_{k}$, with $t_{k}$ the expectation time for several mass hypotheses $k(\pi$, $\mathrm{K}, \mathrm{p}, \ldots$ ) and $t_{\text {event }}$ the event (or starting) time of the collision. The starting time for the ALICE TOF is provided by a weighted average between the measurements from the ALICE T0 detector [41,229] and from the TOF itself [234] (for a track multiplicity larger than 100 , the $t_{\text {TOFevent }}$ is lower than $10 \mathrm{ps}$ ), when both are available. ${ }^{5}$

The detailed calibration procedure consists of mainly three steps: a common offset correction (due to a shift between the LHC clock and the actual collision time), a channel by channel offset adjustment (due to the different path and then different time delays of each channel) and a channel by channel time slewing correction (to disentangle the time from the charge information that is measured via the time over threshold of the signal). Note that a time alignment resolution of about $15 \mathrm{ps}$ is obtained among all channels. A final $56 \mathrm{ps}$ time resolution has been reached in $\mathrm{Pb}-\mathrm{Pb}$ collisions at 5.02 TeV [233], including all the jitter contributions: the intrinsic MRPC time resolution, the electronics and the calibration term. The particle velocity measured by the TOF as a function of the momentum is reported in Fig. 23a. It can be noticed that

\footnotetext{
5 The T0 has a limited acceptance while the $t_{\text {TOFevent }}$ can be used when at least 3 tracks are hitting the detector.
} 


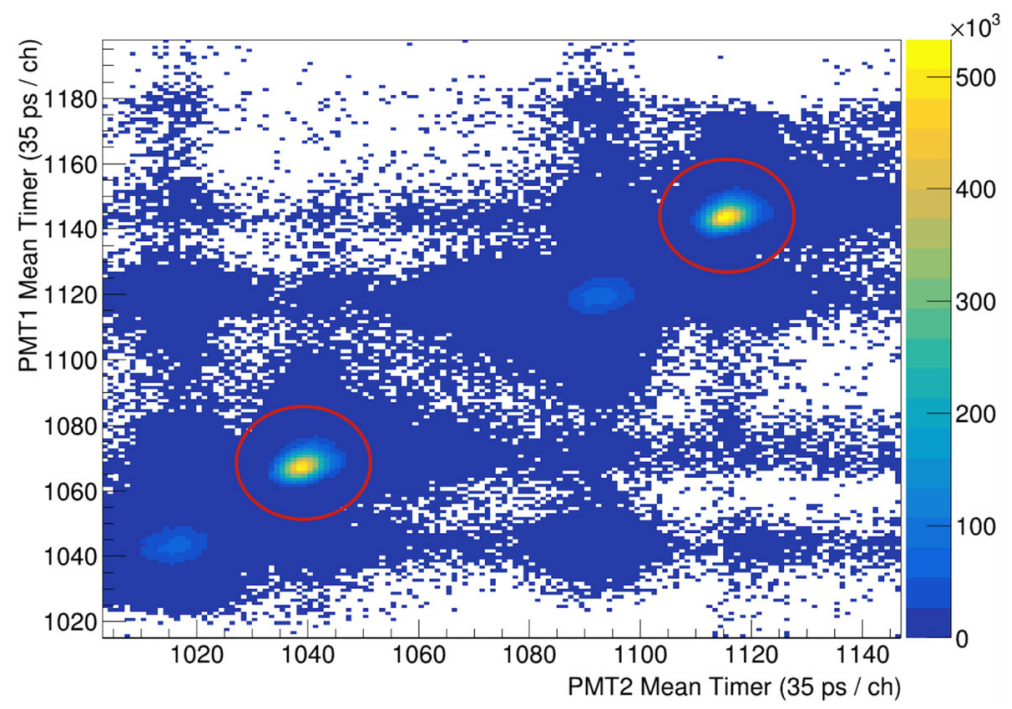

Fig. 24 KAONNIS. MIPs and kaons identification. Time correlation plot of the two PMTs of one of the scintillators: the regions corresponding to kaons (red circle) are clearly separated from MIPs shifted by 23 channels $(0.81 \mathrm{~ns})$. The two replica of this structure are due to the fact that, due to the very high rate of the $D A \Phi N E$ machine RF signal (368 MHz), a RF/2 signal was used as time reference

the electron, pion, kaon, proton and deuteron bands are very well separated at low momentum. This allows a two $\sigma$ separation up to $3 \mathrm{GeV}$ for $\mathrm{K} / \pi$ and up to $5 \mathrm{GeV}$ for p/K. In Fig. 23b an example of the physics analysis applications is reported: the invariant squared-mass distribution has been evaluated from TOF, starting from a selection in the energy loss in the ALICE TPC for pp collisions at $7 \mathrm{TeV}$. A pronounced peak around the anti-deuterium mass is observed, well distinguished from the background [235].

\subsubsection{KAONNIS: kaon identification at DAФNE-LNF}

In $D A \Phi N E$, collisions of $\mathrm{e}^{+} \mathrm{e}^{-}$pairs with a momentum of $510 \mathrm{MeV} / c$ produce $\phi$ mesons almost at rest, which in turn decay into back-to-back $\mathrm{K}^{+}+\mathrm{K}^{-}$pairs with a momentum of $127 \mathrm{MeV} / c$ [30,31]. The low momentum kaons are identified, during the SIDDHARTA experiment, via TOF by the two plastic 1.5 -mm-thick scintillators, placed $6 \mathrm{~cm}$ above and below the interaction point and read at both sides by PMTs. The very good time resolution $(\mathrm{FWHM} \simeq 100 \mathrm{ps})$ of the two plastic scintillators allowed to improve the background rejection using the TOF separation between the kaons and the synchronous background events, mainly due to MIPs produced in time with the machine radiofrequency (RF). This separation is shown in Fig. 24, where the regions corresponding to kaons (red circles) are clearly identified in the correlation plot of the two PMTs of one of the two scintillators.

When the kaons enter the gaseous target, the kaonic atoms are formed and the X-rays emitted during the radiative transitions of the kaonic atoms are then detected by the SDDs (see Sect. 5.2). A triple timing coincidence between the two scintillators and the 
SDDs allows to dramatically reduce the background, reaching an overall background reduction of a factor $\simeq 10^{5}$ (see Fig. 21 ).

The SIDDHARTA-2 experiment plans to perform the kaonic-deuterium measurement in 2021-2022 [36] with an upgraded version of SIDDHARTA that includes a large area, L-shaped scintillators around the target cell, aiming to detect via TOF the pions produced after the $\mathrm{K}^{-}$absorption from the deuterium nuclei.

\subsection{3 n_TOF: identification of neutron-induced elastic reactions}

At n_TOF, a fundamental ingredient for high-precision and accurate measurements of any reaction cross-section is the knowledge of the incident neutron flux as a function of energy. Two counter telescopes (Recoil Proton Telescope, RPT) have recently been developed to extract the neutron flux in the energy region above $10 \mathrm{MeV}$, by exploiting the well known neutron-proton elastic scattering and measuring the recoil nucleus energy via kinematic constraints. Both telescopes consist of a compact trapezoidal structure, placed at a small angle with respect to the neutron beam and pointing to a polyethylene $\left(\mathrm{C}_{2} \mathrm{H}_{4}\right)$ sample, placed in the neutron beam line. The RPTs [236] consist of four independent $\mathrm{BC} 408$ plastic scintillators with increasing thickness: 0.5, 3.0, 6.0 and $6.0 \mathrm{~cm}$, each of them is coupled with a 1" PMT, and one of the two telescopes is supplemented by two stages of silicon detectors.

The selection of signals in temporal coincidence among the different slabs of the telescope, within a window of $10 \mathrm{~ns}$ for the scintillators and $500 \mathrm{~ns}$ for the silicon detectors, guarantees the identification of the events originating only from the polyethylene sample and the suppression of the $\gamma$ and electron background. However, neutrons impinging on the $\mathrm{C}_{2} \mathrm{H}_{4}$ sample can initiate $\mathrm{n}-\mathrm{p}$ scattering as well as $\mathrm{n}+\mathrm{C}$ inelastic reactions which can produce light particles in the final state, i.e. protons, deuterons, tritons, $\alpha$ particles. Therefore, PID capability of the RPTs is crucial to select only protons from elastic scattering and reject background signals. The timing information from RPT $\left(t_{\mathrm{RPT}}\right)$ is referred to the neutron production time in the spallation target and can be related to its energy: the higher value corresponds to a lower energy of the impinging neutron. The measured $t_{\mathrm{RPT}}$ and the multi-stage structure of the RPTs ensure the event selection criteria in terms of number of detectors in coincidence: as the $t_{\mathrm{RPT}}$ decreases the number of the hit detectors increases. As an example, Fig. 25a shows the behavior of the energy deposited in the second scintillator as a function of the $t_{\mathrm{RPT}}$ : events with $t_{\mathrm{RPT}}$ down to $1000 \mathrm{~ns}$, where the secondary particle deposited energy is at the maximum, stop in the second scintillator while at lower $t_{\mathrm{RPT}}$ the secondaries reach the next scintillators and the energy deposited in the second scintillator decreases. It should be noticed that, thanks to the good timing (about $1 \mathrm{~ns}$ ) properties of the scintillators, it is possible to perform the time-to-neutron-energy calibration with a good resolution $\Delta E_{\mathrm{n}} / E_{\mathrm{n}} \sim 10^{-3}-10^{-2}$.

The discrimination between protons and deuterons in the secondaries is then performed using the $\Delta E$ vs $E$ plots, as reported in Fig. 25b for a given $t_{\mathrm{RPT}}$ interval. Because of the kinematic properties of the reaction, protons coming from n-p scattering are identifiable counting only the events localized in the maximum (e.g. the accumulation of events with $\Delta E$ and $E$ of about 12 and $60 \mathrm{MeV}$ respectively). The $\mathrm{n}+\mathrm{C}$ reactions are characterized by a negative $Q$ value, therefore the protons emitted 

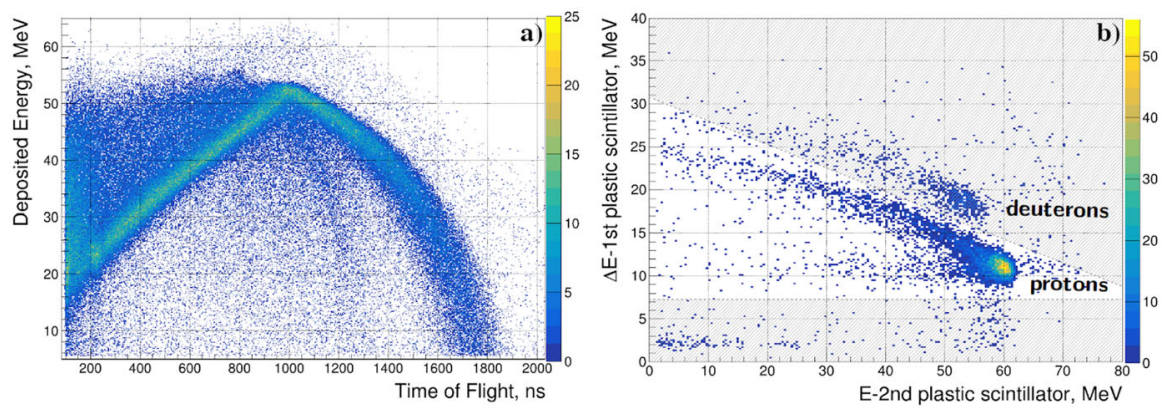

Fig. 25 n_TOF. a Deposited energy in the second scintillator as a function of the time of flight. b $\Delta E-E$ matrices produced by events with a time of flight from 0.965 to $1.04 \mu \mathrm{s}$ hitting the $\mathrm{C}_{2} \mathrm{H}_{4}$ sample. The protons generated by the $\mathrm{n}-\mathrm{p}$ scattering are localized in the peak $(\Delta E \sim 12 \mathrm{MeV}$ and $E \sim 60 \mathrm{MeV})$, which is the characteristic kinematic locus of the reaction

energy distribution has a lower mean value and the kinematic of the nuclear reaction does not produce a peaked distribution in energy populating the tail.

An extensive study through Monte Carlo simulations with neutrons impinging on a realistic setup was performed to estimate the performances of the techniques used in the analysis [237]. The correlation between $\Delta E-E$ and TOF enables a selection of proton events at the level of $1 \%$ accuracy, for protons with energy up to $180 \mathrm{MeV}$. Above this energy, protons do not stop anymore inside the telescope therefore the techniques $\Delta E-E$ and TOF- $E$ need to be combined with other strategies to disentangle the contribution of protons from all background events.

The above-described PID techniques are presently being applied for the first time in the ${ }^{235} \mathrm{U}(\mathrm{n}, \mathrm{f})$ analysis, and pave the way for the study of neutron-induced reactions above $20 \mathrm{MeV}$ of neutron energy.

\subsection{Nuclei identification in energy range of the order of $10 \mathrm{MeV} /$ nucleon}

\subsubsection{ASFIN: $\alpha$ identification to define elastic and inelastic interactions}

A nuclear cluster is defined as a group of nucleons, collectively acting as a single particle whose internal degrees of freedom can be neglected, such as $\alpha$ particles [238]. A very useful method to study this kind of structures is represented by the Thick Target in Inverse Kinematics (TTIK) [239]. Briefly, in TTIK resonant scattering, a low-intensity beam is incident on an extended gas target. As the projectiles slow down in the gas, elastic scattering reactions across a wide range of center-of-mass (c.m.) energies can take place (corresponding to a range of depths within the gas) allowing to measure large-range excitation functions in one irradiation with the same initial beam energy. A light recoil created in an elastic scattering event of the beam particle on the nucleus of the target gas can easily penetrate further through the gas and be detected by an appropriate array of detectors, while the other fragments remain in the gas target. 


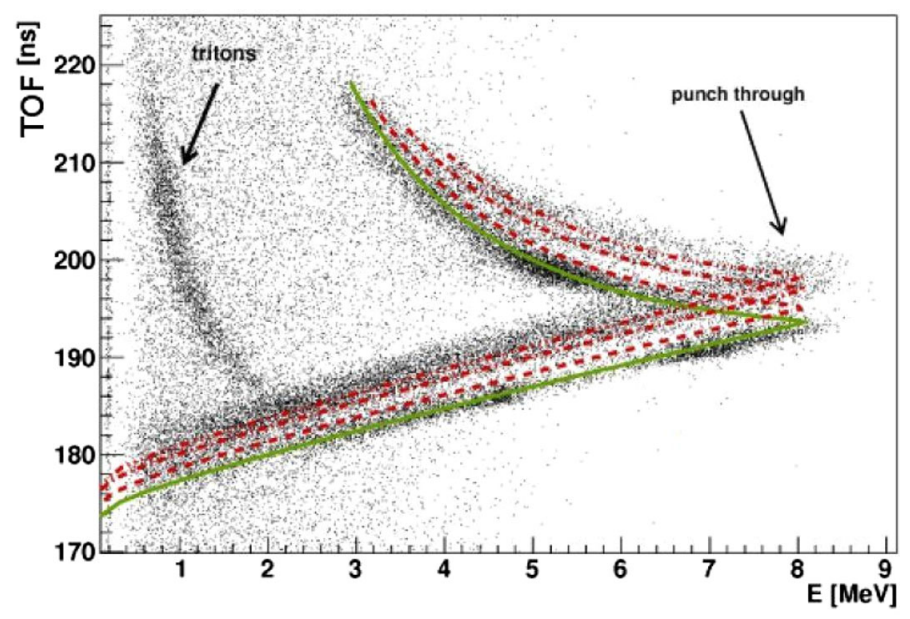

Fig. 26 ASFIN. Experimental detected energy in DSSSD versus TOF plot. Since inverse time logic has been used, large time intervals $(\approx 220 \mathrm{~ns})$ correspond to events which occur at the entrance of the chamber, while smaller intervals $(\approx 175 \mathrm{~ns})$ correspond to events which take place closer to the detectors. The $\alpha$ particle punch-through energy is around $8 \mathrm{MeV}$. In the picture the green full lines represent loci for elastic scattering $\alpha$-particles events, while the dashed red lines represent the ones for the inelastic $\alpha$ related to the first three states of ${ }^{8} \mathrm{Li}$ at an excitation energy of $\mathrm{E}_{x}=0.9808,2.255,3.21 \mathrm{MeV}$, respectively. Figure adapted from [240]

An experimental limitation of using the TTIK method is represented by light particles coming from other possible reaction mechanisms, such as inelastic scattering [240]. Indeed, only by analyzing the particle energy deposition spectra, it is not possible to identify the reaction mechanisms for the particles production. For this reason, it was decided to improve the TTIK method by introducing the TOF measurement since the measured energy of a detected light particle depends on the reaction process in which it was produced, as well as on the position in the gas target where the reaction has occurred. Therefore, the TOF measurement allows to discriminate elastic from inelastic reaction events.

This technique was successfully applied to support the picture of $\alpha{ }^{8} \mathrm{Li}$ cluster configurations for excited states in ${ }^{12} \mathrm{~B}$ by means of the measurement of ${ }^{8} \mathrm{Li}+{ }^{4} \mathrm{He}$ resonant elastic scattering. The ${ }^{8} \mathrm{Li}$ beam was produced by the radioactive beam facility EXCYT at LNS [241] and delivered into a scattering chamber filled with ${ }^{4} \mathrm{He}$ gas at a pressure of 700 mbar [240]. The stop signal for the TOF measurement is provided by a micro channel plate (MCP), placed before the entrance of the scattering chamber, while the start signal is given by a $500 \mu \mathrm{m}$ DSSSD, in an inverse time logic. The DSSSD also provides the energy information. The resulting energy versus TOF plot is shown in Fig. 26 where the calculation for $\alpha$ particles that are elastically (solid green line) or inelastically scattered (short-dashed red lines) are superimposed on the experimental data, proving that TOF measurements allowed for the discrimination between elastic and inelastic scattering [240]. 
Recently, the TTIK method with TOF measurement opened the possibility of studying exotic cluster structures in nuclei, of relevance for both nuclear physics and nuclear astrophysics [242].

\subsubsection{CHIRONE: light and heavy nuclei identification with silicon detectors}

The TOF technique is extensively used in the CHIMERA apparatus for direct velocity measurements of ions with $Z>2$ and for mass determination of particles stopped in the Si detectors [45,243]. This is realized thanks to the long base-of-flight, in the range of 1-3.5 m, for the 688 telescopes of the forward part of the apparatus. Time of arrival of particles in the detectors is measured by a $30 \%$ constant fraction discrimination (CFD) acting on silicon signal, and a $\Delta t$ time interval is measured with respect to a common stop given by the RF signal for beams delivered by the LNS Cyclotron. An example of correlation between the energy loss in a silicon detector, $\Delta E$, and $\Delta t$ is given in Fig. 27a for the reaction ${ }^{124} \mathrm{Sn}+{ }^{64} \mathrm{Ni}$ at $20 \mathrm{MeV} /$ nucleon. The slower particles stopped in the silicon detector are the ones sitting in the left zone of the plot ( $\Delta t<480 \mathrm{~ns}$ ). In this case, $\Delta E$ is simply the kinetic energy of ions and in the $\Delta E-\Delta t$ plot the lines correspond to different masses. Whereas, particles passing through silicon detectors are the ones sitting in the right part of the plot $(\Delta t>480 \mathrm{~ns})$. In this case, lines in the plot correspond to different $Z$. Therefore, the region at $\Delta t>480 \mathrm{~ns}$ contains information only on the $Z$ of particles, that is already attainable by the $\Delta E-E$ method (see 3.2.1). The identification in mass needs to correct the measured $\Delta t$ for the effects related to $Z, A$ and $E$ dependence of the time profile of the signals, due to plasma delay effects, and for the time-charge walk effect, for low amplitude signals. Mass determination is performed through a semi-automatic and iterative procedure, and parameters describing $\Delta t$ correction for the above mentioned effects are obtained by a fit. As an example, the inset of Fig. 27a shows the masses of selected loci corresponding to $A=7,9,11,13$ and 15 , as a function of $\Delta E$, as obtained using the $\Delta t$ correction functional form. The typical resolution obtained is $\Delta A / A \sim 1 / 20$ and effective thresholds for mass determination are around $5 \mathrm{MeV}$.

The collaboration is also involved in experiments with radioactive ions beams (RIBs), using the FRIBs facility at INFN-LNS with the in-flight fragmentation method [4]. The fragmentation reaction produces several stable and unstable isotopes at the same time. Optical elements are used to select and to transport the exotic beam. However, a magnetic selection does not allow for the separation of isotopes with the same $m / q$ ratio. For this reason, it is necessary to proceed with the characterization of beam components, event-by-event, to select the isotope of interest for the specific study off-line. In the CHIMERA beam line the identification takes place through the $\Delta E$-TOF method, using a tagging system, specifically implemented and developed [244], that allows to measure event-by-event the isotopic composition of the exotic beam. This tagging system consists of a Micro-Channel Plate detector (MCP) and of a DSSSD. The start signal is given by the MCP, located at a distance of $12.9 \mathrm{~m}$ from the DSSSD. The stop signal and the $\Delta E$ are provided by the strips of the DSSSD, mounted about $2 \mathrm{~m}$ before the reaction target. Time resolutions around $500 \mathrm{ps}$ are reached. Figure $27 \mathrm{~b}$ shows the $\Delta E$-TOF plot obtained with the tagging system, using a fragmentation reaction of a stable ${ }^{70} \mathrm{Zn}$ beam accelerated at $40 \mathrm{MeV} /$ nucleon 

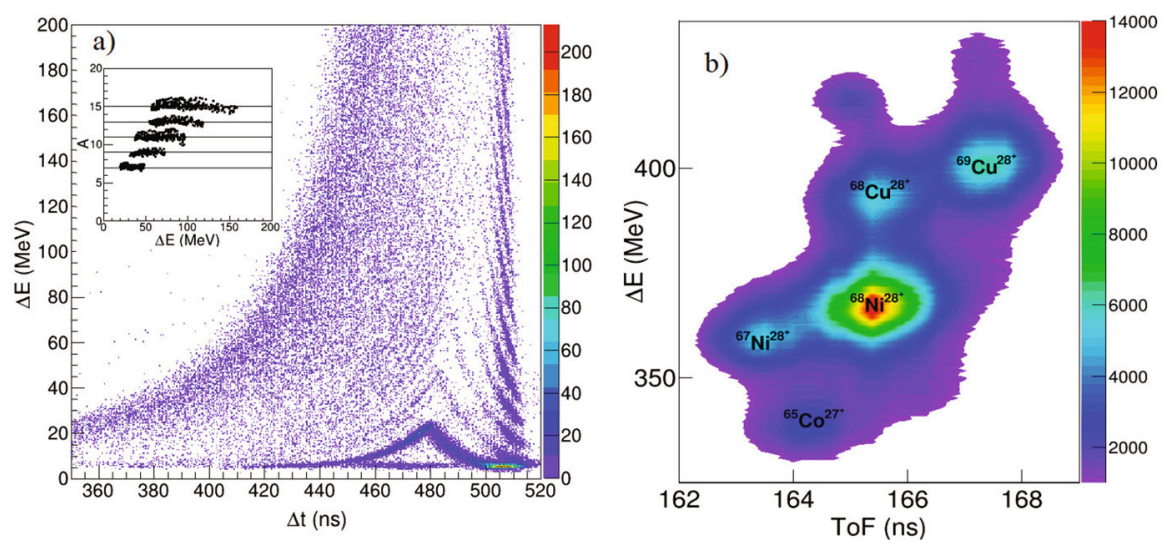

Fig. 27 CHIRONE. a Energy loss in a silicon detector $(\Delta E)$ vs "time-of-flight" for the reaction ${ }^{124} \mathrm{Sn}+$ ${ }^{64} \mathrm{Ni}$ at $20 \mathrm{MeV} /$ nucleon, as typical for a CHIMERA telescope placed around $\theta=10.75^{\circ}$ and at $180 \mathrm{~cm}$ from target. The inset shows the reconstructed atomic mass number as a function of $\Delta E$ for selected loci corresponding to given isotones $(A=7,9,11,13$ and 15), as resulting from the identification procedures. b $\triangle E$-TOF plot obtained with the tagging system of the multidetector CHIMERA, using a fragmentation reaction of a stable ${ }^{70} \mathrm{Zn}$ beam accelerated at $40 \mathrm{MeV} /$ nucleon and a ${ }^{9} \mathrm{Be} 250 \mu \mathrm{m}$ target (figure adapted from Ref. [144])

and ${ }^{9} \mathrm{Be}(250 \mu \mathrm{m})$ target [144]. At this energy, the probability to produce not fully stripped ions is significant. It was indeed interesting to measure the presence of different charge states, after the ${ }^{9} \mathrm{Be}$ production target. The population was evaluated changing the magnetic field of the optical elements and measuring the beam current of the various charge states obtained. This experiment represents an upper limit for the FRIBs performance and a point of reference for the tagging system of the future FraISe facility at LNS [4].

\subsubsection{FOOT: particle identification for heavy fragment cross section measurements with E-TOF method}

As mentioned in Sect. 2.5.1, in the FOOT setup focusing on detecting high $Z$ fragments the identification of the charge $Z$ is performed exploiting the measurement of the TOF and the energy released $\Delta E$ in a thin wall of plastic scintillators TOF-Wall (TW), while the momentum $p$ from the magnetic spectrometer and the kinetic energy $E_{k}$ measured in the calorimeter provide the isotopic identification.

The fragment charge identification via the $\Delta E$-TOF method is based on the signals provided by a start counter (SC) and the TW. The SC [245] is the first detector encountered by the beam, providing the particle rate and the crossing time of the primary particles. It is a thin $(250 \mu \mathrm{m})$ foil of plastic scintillator material with an active area of $5 \times 5 \mathrm{~cm}^{2}$. The TW [246,247], located about $1 \mathrm{~m}$ behind the target, measures the energy loss $\Delta E$ of the fragments and provides their arrival time. It consists of 40 bars of plastic scintillator material, arranged in two orthogonal layers of 20 each. Each bar has an active area of $44 \times 2 \mathrm{~cm}^{2}$ and is $3 \mathrm{~mm}$ thick. The light produced in the bars is collected at each end by four silicon photomultipliers. Fragment charge 
Table 1 FOOT: TOF, energy and $Z$ resolutions obtained from the data for various projectiles

\begin{tabular}{llllll}
\hline Facility & Particle & $E_{\text {beam }}[\mathrm{MeV} /$ nucleon $]$ & $\sigma(\mathrm{TOF})[\mathrm{ns}]$ & $\sigma(E) / E[\%]$ & $\sigma(Z)[\%]$ \\
\hline CNAO & $\mathrm{p}$ & 60 & 0.265 & 5.72 & 6.10 \\
$\mathrm{CNAO}$ & ${ }^{12} \mathrm{C}$ & 115 & 0.054 & 4.00 & 2.51 \\
$\mathrm{CNAO}$ & ${ }^{12} \mathrm{C}$ & 260 & 0.066 & 4.73 & 3.52 \\
$\mathrm{CNAO}$ & ${ }^{12} \mathrm{C}$ & 400 & 0.074 & 4.70 & 3.85 \\
GSI & ${ }^{16} \mathrm{O}$ & 400 & 0.076 & 5.19 & 2.67 \\
\hline
\end{tabular}

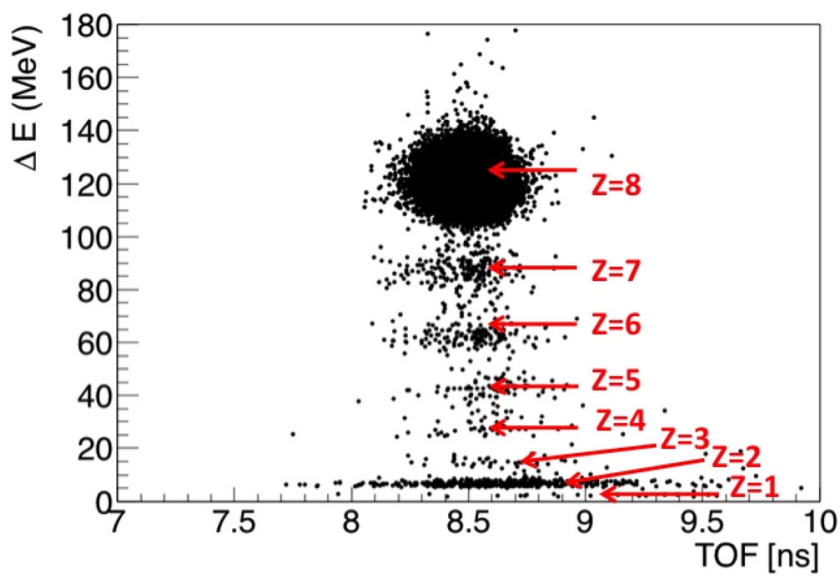

Fig. 28 FOOT. Measured distribution of $\Delta E$ versus TOF of the fragments produced during the irradiation of a 5-mm-thick carbon target with $400 \mathrm{MeV} /$ nucleon oxygen ions. The various nuclei atomic numbers can be distinguished

discrimination is achieved by correlating the energy $\Delta E$ deposited in the bars with the measured TOF.

Table 1 reports the TOF, energy and $Z$ resolutions obtained during test beam acquisitions at the CNAO (Pavia, Italy) and GSI (Darmstadt, Germany) facilities for various projectiles. The Z-resolution for carbon and oxygen ranges from 2.5 to $3.9 \%$, compatible with the FOOT requirements. The resolution for protons is $6.1 \%$, however the emulsion chambers (see Sect. 3.4) are expected to perform better for such low $Z$ nuclei.

In Fig. 28, the $\Delta E$ versus TOF distribution obtained from irradiation of a 5-mmthick carbon target with a $400 \mathrm{MeV} /$ nucleon oxygen beam during a test beam at GSI is reported. Further improvements to the TW are foreseen in the near future, with a new mechanical frame and a better optical insulation of the bars.

Regarding the mass $\mathrm{A}$ of the fragments, this will be determined combining $\beta, p$ and $E_{k}$, using the TOF, the tracking system and calorimeter, respectively. The mass will be extracted using a $\chi^{2}$ minimization approach, and using the Augmented Lagrangian Method (ALM) [248]. The construction of all components of the FOOT detector is being finalized. New data, including not only $Z$ but also A determination, are expected for 2022 at CNAO and GSI, where the full setup will take data. 


\subsubsection{FORTE: separation of fusion-fission and quasi-elastic events}

The mass and total kinetic energy (TKE) of two binary-reaction fragments are reconstructed using the so-called two-velocity $(2 \mathrm{~V})$ method, namely, by measuring their velocity vectors in coincidence [249]. The FORTE collaboration makes use of a TOF spectrometer consisting of 4 arms, each one containing a start and a stop detector. The conceptual design is based on the CORSET one [249]. The stop detectors are position sensitive. The information on the time of flight and on the position of the fragments in each respective arm along with the two-body kinematics allows the reconstruction of the mass and kinetic energies of the fragments. Two pairs of arms placed on opposite sides with respect to the beam axis on the same reaction plane constitute the basic element. It is then possible to span a folding angle ${ }^{6}$ between $80^{\circ}$ and $140^{\circ}$, namely, a larger mass asymmetry of the produced fragments.

The long measurements of highly ionizing particles (large doses) require detectors with a high radiation hardness and stability. Hence, start and stop detectors are built using two MCPs chevron assembled. The stop detector consists of a conversion entrance foil upstream of two MCPs (the fragment is stopped inside the MCPs) and provides a timing stop signal and two coordinates determined via delay lines. It has an active area of $70 \times 90 \mathrm{~mm}^{2}$ to cover the solid angle of the start detector. The flight path is $30 \mathrm{~cm}$ long. A coincidence of two opposite arms with stop signals and at least one start signal usually defines a trigger. Each arm provides a spatial and time resolutions of about $1 \mathrm{~mm}$ and $180 \mathrm{ps}$ (full width at half-maximum), respectively.

The primary mass and energy of binary fragments are reconstructed in an iterative procedure that considers the fragment-velocity vectors, momentum and nucleon conservation laws, and corrections due to heavy fragment energy losses in different media. Then, the TKE of reaction products is determined as the sum of the fragment energies in the center-of-mass system.

The result of this procedure and the corresponding raw TOF-TOF matrix for the ${ }^{32} \mathrm{~S}+{ }^{109} \mathrm{Ag}$ reaction at $180 \mathrm{MeV}$ are shown in Fig. 29. The three clusters in TOFTOF matrix well identify the products of fusion-fission (FF) and quasi-elastic (QE) collisions. In case of FF events, the symmetric split produces the central cluster with equal mean TOF values registered in left and right CORSET arms. The other two clusters are produced by QE events. Here the TOF values of projectile-like fragments are small and narrowly distributed, while those of target-like fragments are large and broadly distributed. The fragment mass-TKE distributions represent the most sensitive observables to improve the knowledge of the reaction dynamics. These quantities are used to cleanly separate the products of competing processes, to estimate the intensity and nature of nuclear viscosity and measure the contribution of different fission modes [250].

The ongoing upgrade involves larger angular coverage, position sensitive passingthrough stop detectors, Bragg chambers for $\mathrm{Z}$ identification, new electronics for pulsed beams/higher rates. A foreseen application concerns the study of exotic nuclei located in the Terra Incognita of the nuclear chart produced via multinucleon transfer reactions.

\footnotetext{
6 The folding angle is the angle between the directions of the two coincident fragments, and it is a quantity generally used to differentiate between partial and full momentum transfer collisions.
} 

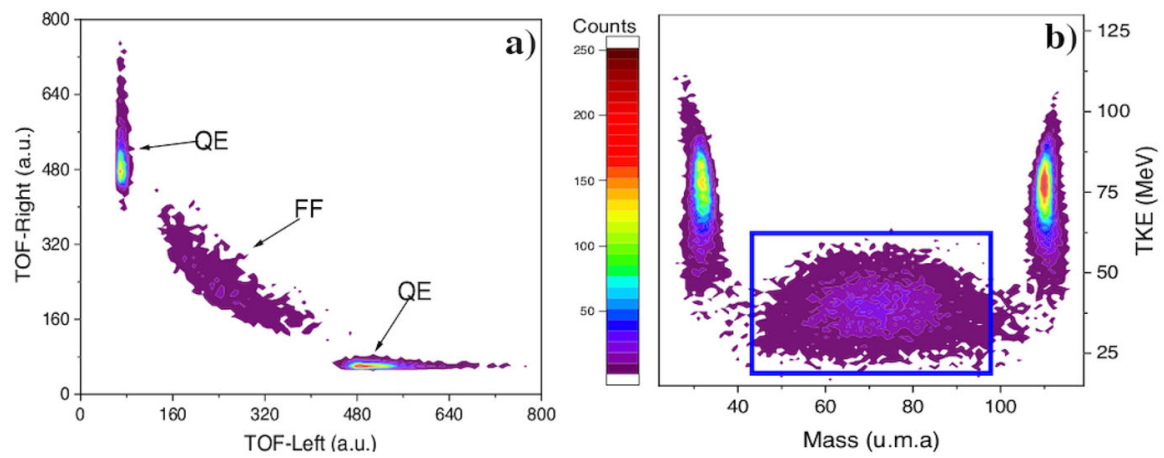

Fig. 29 FORTE. TOF matrix (a) and Mass-TKE distribution (b) of the binary fragments measured in coincidence using two opposite TOF arms in the ${ }^{32} \mathrm{~S}+{ }^{109} \mathrm{Ag}$ reaction at beam energy of $180 \mathrm{MeV}$. Quasielastic (QE) and fusion-fission (FF) events are well separated in TOF matrix. The fission-like fragments are inside the blue rectangle in the Mass-TKE matrix. The mass and energy resolutions of the spectrometer are 3 a.m.u. and $5 \mathrm{MeV}$, respectively

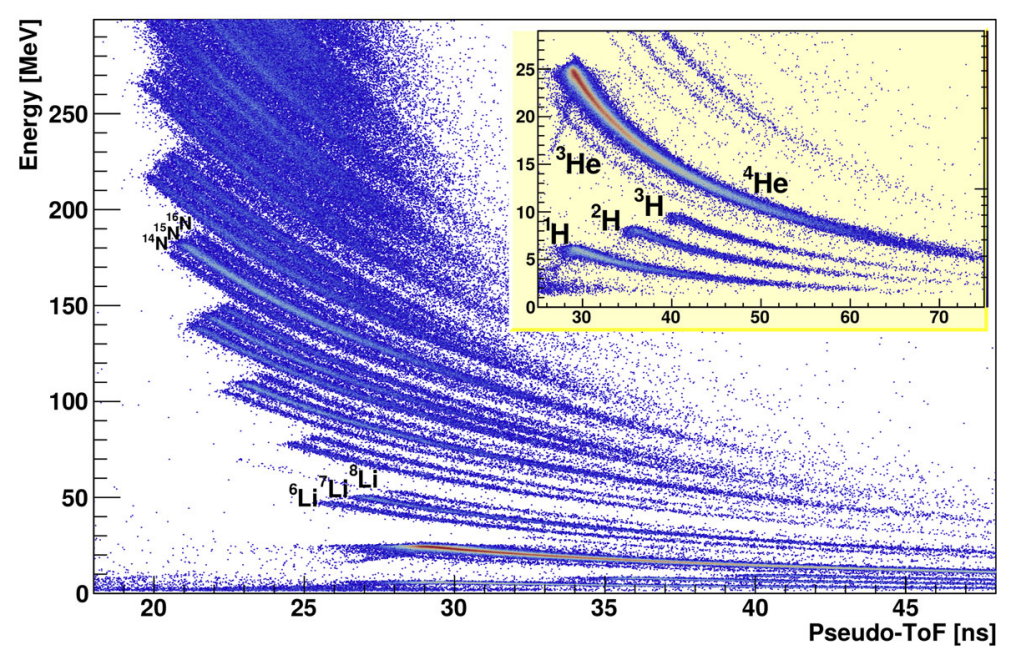

Fig. 30 NUCLEX. $E-T O F$ correlation for a FAZIA silicon detector. TOF measurement is affected by the signal rise time walk, thus the correlation shows a dependence on $Z$ and not only on particle mass. Data comes from ${ }^{48} \mathrm{Ca}+{ }^{12} \mathrm{C}$ reactions at $25 \mathrm{MeV} /$ nucleon (adapted from [251])

\subsubsection{NUCLEX: identification of charged products fragments with silicon detectors}

In TOF measurements, when it is not possible to install a start detector or the beam is not pulsed, the NUCLEX collaboration has developed a new method to recover the start time mark. First, a fully identified reference ion is selected and it is used to calculate the interaction time. This is done knowing the flight base $d_{\text {ref }}$, the kinetic energy $E_{\text {ref }}$ (well measured by silicon sensors) and the mass $m_{\text {ref }}$ (from $\Delta E-E$ or PSA correlations, see 4.1.2). 
Once the time of the interaction is obtained, the timing of all other particles is referred to it as a pseudo-TOF measurement, as described in detail in a recent FAZIA work [251]. The TOF signal is provided by the silicon sensors of the detector telescopes, with an intrinsic time resolution around 500 ps for the nuclei under investigation. An example of the energy-time correlation obtained with this procedure is shown in Fig. 30 for particles stopped in the first silicon layer.

It should be noted that reverse mounted silicon detectors have rather slow signals (rise times from 10 to $100 \mathrm{~ns}$ ): this feature is useful to better exploit the pulse shape discrimination technique (see Sect. 4.1.2), but affects the pseudo-TOF measurement. In fact, the rise time strongly depends on $Z$, so we observe also a $Z$ (and not only $A$ ) separation in the $E$-pseudo-TOF correlation ridges (Fig. 30). For example it is possible to distinguish ${ }^{3} \mathrm{H}$ and ${ }^{3} \mathrm{He}$, or ${ }^{10} \mathrm{Be}$ and ${ }^{10} \mathrm{~B}$. However, on the other side, the degraded time resolution limits the present $Z$ range to about 10 .

Overall, in FAZIA, (mounted with a flight base of $1 \mathrm{~m}$ ) the identification with timing allows to achieve A discrimination from $Z=1$ to 10 , opening the door to the study of light particles emitted by quasi-target fragments in deep inelastic collisions.

\subsubsection{PRISMA: heavy nuclei identification with a magnetic spectrometer}

PRISMA is a magnetic spectrometer installed at LNL, optimized for ions in the mass range $A=20-200$ and energies of 2-10 MeV/nucleon, well suited for the beams provided by the Tandem-ALPI-PIAVE accelerator complex [252]. It is characterized by the large solid angle coverage $(\approx 80 \mathrm{msr})$, momentum acceptance of $\pm 10 \%$, mass resolution of $1 / 300$ and energy resolution up to $1 / 1000$ (exploiting TOF measurement) [253]. In addition, the spectrometer can be aligned over a wide angular range from $-20^{\circ}$ to $130^{\circ}$ with respect to the beam line.

The ion trajectories are reconstructed from the target to the focal plane detectors event-by-event, using an algorithm that takes into account detailed magnetic field maps, measured entrance and exit positions of the particles, the time of flight and the geometry of the instrument [254]. Ions produced in the target go through an entrance detector consisting of microchannel plates, providing two-dimensional position information and the start signal for the time of flight [255]. Then, a quadrupole magnet focuses incoming ions in the vertical plane before they enter the dipole magnet. When crossing the dipole, ions with different momentum-charge ratio follow different trajectories. Therefore, tuning the dipole magnetic field it is possible to detect a specific range of nuclear products on the focal plane of the magnet.

The detection system at the focal plane consists of a set of multi-wire parallel plate avalanche counters (MWPPAC), providing the two-dimensional arrival position of ions and the stop signal for the time of flight (with a typical time resolution of about $300 \mathrm{ps}$ ), followed by an array of ionization chambers for energy loss and total energy measurements, used to derive the atomic number of reaction products. Detailed descriptions of the focal plane detectors can be found in [253].

Typical performances of the spectrometer in terms of detection efficiency and resolution are displayed in Fig. 31, which shows the correlation mass-charge ratio vs. position in the MWPPAC detector and the yields for multi-nucleon transfer reaction channels populated in the reaction ${ }^{40} \mathrm{Ar}+{ }^{208} \mathrm{~Pb}$ with a ${ }^{40} \mathrm{Ar}$ beam at $E_{\text {lab }}=260 \mathrm{MeV}$ [257]. 

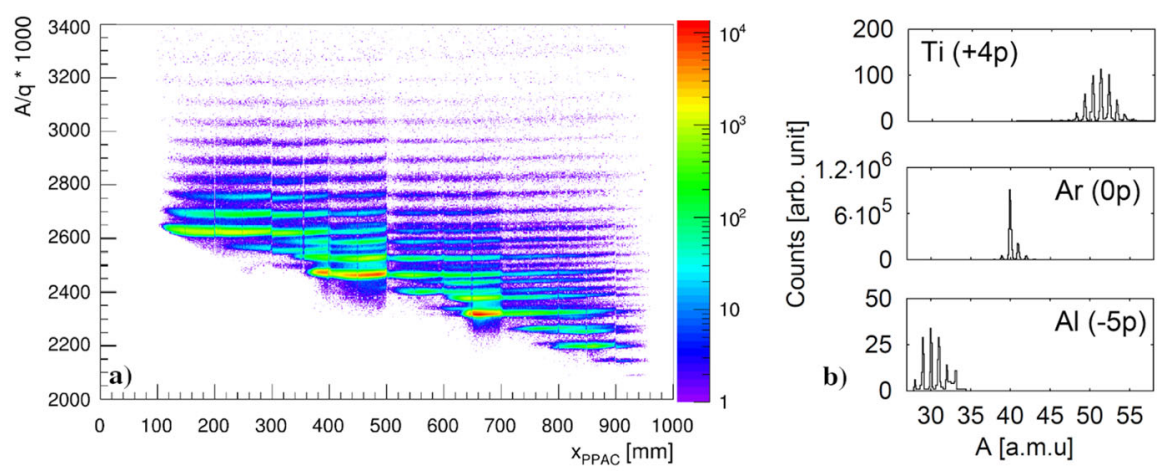

Fig. 31 PRISMA. a Mass-charge ratio vs. horizontal position correlation in the MWPPAC focal-plane detector. Ions with higher momentum-charge ratio are less deflected in the dipole magnetic field. Nuclear charge is derived from the energy loss inside the ionization chamber. b Examples of obtained mass spectra for channels corresponding to different proton pick-up and stripping channels (here pick-up and stripping are referred to the light partner of the reaction). All proton-stripping reaction channels from $(+4 p)$ to $(-5 p)$ were identified. Both plots refer to the results obtained with the reaction ${ }^{40} \mathrm{Ar}+{ }^{208} \mathrm{~Pb}$ at $E_{\text {lab }}=260 \mathrm{MeV}$ (adapted from [256])

Recently, the spectrometer was equipped with a second arm used to identify the fragments produced in low energy heavy-ion nuclear reactions and reconstruct the kinematics of binary reactions [258]. The second arm consists of a position sensitive MWPPAC followed by a Bragg Chamber. The performances of the second arm have been evaluated in experimental conditions using different beam-target systems [91, 258]. The position resolution of the MWPPAC was established to be $1 \mathrm{~mm}$ in both $X$ and $Y$ directions, while the energy and atomic number resolution of the Bragg chamber were found to be $\Delta E / E \sim 1 \%$ and $\Delta \mathrm{Z} / \mathrm{Z} \sim 1.7 \%$.

\section{Calorimeters and muon identification}

In this section, the identification capabilities of experiments using the calorimetric technique or spectrometers for muon selection are described. Various detectors designed to measure the total energy of particles have been already described in this review, but, as the energy increases, more sophisticated solutions must be developed. Calorimetry has become the natural option and allowed not only the possibility to measure the energy of single particles, but also the global energy of groups of particles (jet studies) or events with missing energy (a signature of specific processes). Indeed, the specific role of calorimeters is the measurement of the energy of a particle by completely absorbing its momentum through interaction with passive layers interleaved with sensitive layers (segmented calorimeters) or using a unique sensitive volume (homogeneous design): in all cases the given material is best adapted to the development of an electromagnetic shower or the containment of hadronic processes. It is common that the energy information is supported by angle, position or time information. A plethora of implementations have appeared, the specific design depending on the kind and the energy of the particles to be investigated: given that the resolution 
improves with energy, the achievable shower signal and the electronic noise must be tuned to minimize the associated uncertainties.

In the following three experiments, in alphabetical order which happens to be of decreasing beam energy, present their calorimeters in three very different experimental contexts and four specific goals, witnessing to the robustness and the wide diffusion of the principle. The energy of the particles to be detected determines of course the thickness of the material to be traversed. In the first example, the BGOOD experiment (of the MAMBO collaboration), the detector has also high granularity (about $20 \mathrm{msr}$ per detector element) to keep the angular resolution good enough for detection of photons coming from meson decay. In the second case, JLAB experiment, the focus is on electron detection in the forward direction, with emphasis on time and energy resolution and two complementary detectors for the photon-electron separation and electron tracking. The last calorimeter presented is from ALICE, which has the specific goal to measure high momentum photons and electrons and jets, opportunities expanded by the addition at a later stage of further modules. An example of muon spectrometer has been added that, while not belonging properly to the PID class of detectors, it is ipso facto a method to select muons over a huge background of hadrons and leptons, using the standard technique of absorbing all the other particles in a dense material.

\subsection{ALICE: electrons, photons and muons tagging in a high-multiplicity, high-energy environment}

\subsubsection{Electromagnetic calorimeter}

The ElectroMagnetic Calorimeter (EMCal) [41,259] is one of the central barrel ALICE detectors and it is based on a Pb-scintillator sampling technique. It is located at $4.5 \mathrm{~m}$ from the beam axis and it has a cylindrical coverage of $|\eta|<0.7$ and $\Delta \Phi=107^{\circ}$. Since 2015 the EMCal acceptance has been enlarged in azimuth with DCal, an additional set of modules (DCal: $0.22<\eta<0.7 \Delta \Phi=67^{\circ}$ ), opening the possibility to study di-jets. In total EMCal and DCal have 17,644 cells, consisting of towers of $6.0 \times$ $6.0 \times 24.6 \mathrm{~cm}^{3}$, corresponding to 20.1 radiation lengths. The plastic scintillators have longitudinal wavelength shifting fibers to collect the light which are read by Avalanche PhotoDiodes (APDs).

The EMCal can identify electrons and provide trigger on jets, high-momentum photons and electrons. To distinguish between electrons and charged hadrons, it uses the $E / p$ method, with $p$ measured by the central ALICE-TPC: the EMCAL measures the energy deposition and compares it to the measured momentum. Indeed, while electrons deposit their full energy in the calorimeter $(E / p \approx 1)$, hadrons tend to lose only a small fraction. In Fig. 32a, the $E / p$ distribution for $\mathrm{Pb}-\mathrm{Pb}$ collisions at $\sqrt{s_{\mathrm{NN}}}=5.02 \mathrm{TeV}$ is shown. A clear separation between hadrons and electrons is visible. The separation between electrons and hadrons is further increased considering the different shape of the shower. When two clusters overlap partially, the shower cross section assumes an elliptical shape, whose long axis is characterized by the $\sigma_{\text {long }}^{2}$ parameter. As an example of application, in the standard analysis, the neutral pions production, in $\mathrm{Pb}-\mathrm{Pb}$ collisions at $\sqrt{s}=2.76 \mathrm{GeV}$ the mass width is $\approx 10 \mathrm{MeV} / c^{2}$ 


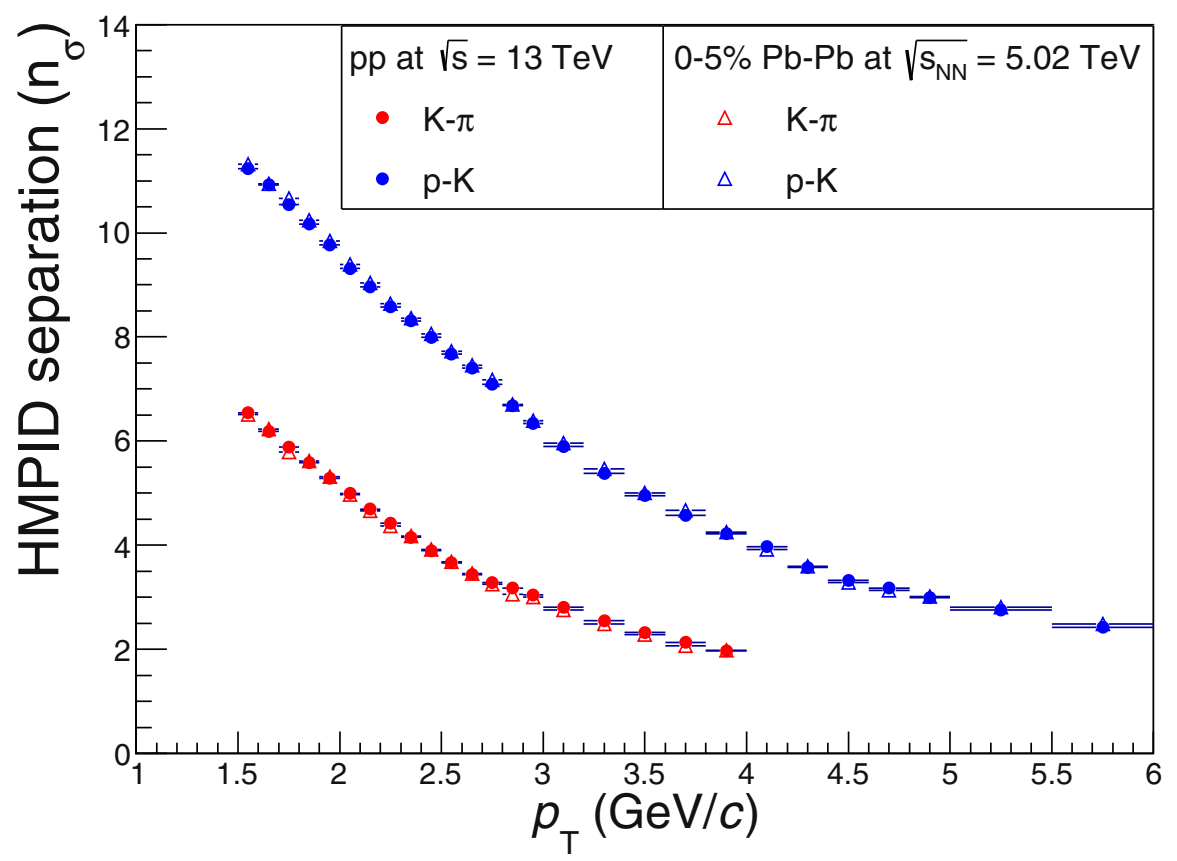

Fig. 32 ALICE. a Distribution of electron energy-over-momentum $E / p$ ratio. b Electron identification purity in central, $0-10 \%, \mathrm{~Pb}-\mathrm{Pb}$ collisions at $\sqrt{s_{\mathrm{NN}}}=5.02 \mathrm{TeV}$ ( from [259])

at $p_{\mathrm{T}}=5 \mathrm{GeV} / c$, with an upper limit on $p_{\mathrm{T}}$ of $20 \mathrm{GeV} / c$ due to the cluster size, which sets the minimum photons relative angle. However, analysing the shape of the electromagnetic shower defined above in analogy with the electron-hadron separation, it is possible to measure neutral pions up to $200 \mathrm{GeV} / \mathrm{c}$ with a purity of $81-89 \%$ in pp collisions, decreasing with $p_{\mathrm{T}}$ (see [260]). In Fig. 32b, the electron identification purity in central $\mathrm{Pb}-\mathrm{Pb}$ collisions is reported. Together with electron identification in the ALICE TPC, the EMCal detector allows the reconstruction and identification of electrons up to $p_{\mathrm{T}}=30 \mathrm{GeV} / c$.

\subsubsection{Muon spectrometer}

The ALICE muon spectrometer covers the pseudorapidity region $2.5<\eta<4$; it consists of a front hadron absorber, followed by a set of five tracking stations, a dipole magnet, an iron wall acting as muon filter and two trigger stations [40].

The absorber downstream the interaction point corresponds to 10 interaction lengths $\left(\lambda_{I}\right)$. Ten multi-wire proportional chambers arranged in five stations provide the space points to reconstruct the muon trajectories, bent by a $0.7 \mathrm{~T}$ magnetic field generated by means of a $5 \mathrm{~m}$ long dipole magnet. An iron wall of $7.2 \lambda_{\mathrm{I}}$ filters the residual hadrons and muons with a momentum approximately greater than $4 \mathrm{GeV} / c$, to reduce the background due to pion and muon decays which survived the front absorber. It is placed between the tracking chambers and the trigger resistive plate chambers (RPCs), also used for tracking. 

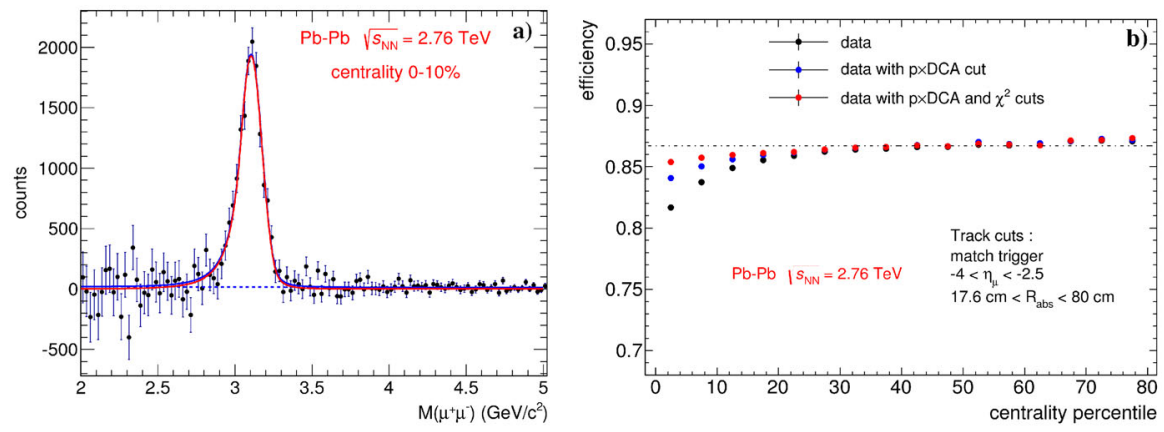

Fig. 33 ALICE. a Dimuon invariant mass spectrum in $0-10 \%$ most central $\mathrm{Pb}-\mathrm{Pb}$ collisions at $\sqrt{s_{\mathrm{NN}}}=2.76 \mathrm{TeV}$, with fit to the $J / \psi$ peak by a Crystal Ball function (from Ref. [41]). b Measured muon track reconstruction efficiency in $\mathrm{Pb}-\mathrm{Pb}$ collisions as a function of the collision centrality

The ALICE muon spectrometer is used to study the light vector mesons $(\rho, \omega, \phi)$ and quarkonia $(J / \psi$ and $\Upsilon$ families) in their dimuon decay channel, and to measure the production of single muons from decays of heavy-flavour hadrons and $\mathrm{W}^{ \pm}, Z$ bosons.

Track reconstruction in the muon spectrometer is based on a Kalman filter algorithm [261,262]; the tracks are then extrapolated to the vertex position measured by the Inner Tracking System (the inner six layer of silicon detectors [40], see Sect. 3.3) and a correction for energy loss and multiple Coulomb scattering in the absorber is applied [262].

Figure 33a shows the dimuon invariant mass spectrum, after background subtraction, in the $0-10 \%$ most central $\mathrm{Pb}-\mathrm{Pb}$ collisions at $\sqrt{s_{\mathrm{NN}}}=2.76 \mathrm{TeV}$, with the fit to the $J / \psi$ peak by an extended Crystal Ball function [263]: the residual background is negligible and the mass resolution at the $J / \psi$ peak is around $\sim 73 \mathrm{MeV} / c^{2}$ [41].

The muon detection efficiency (see Fig. 33b) shows a drop for central $\mathrm{Pb}-\mathrm{Pb}$ collisions $(<20 \%)$, largely due to fake tracks and that can be strongly reduced applying an additional cut on both the $p \times$ DCA (momentum $\times$ distance of closest approach) and the normalized $\chi^{2}$ of the tracks $\left(\chi^{2}<3.5\right)$ [41].

The mass resolution at low masses is limited by multiple scattering inside the front absorber. This effect will be mitigated by the insertion of a silicon pixel detector (the Muon Forward Tracker), which will be placed upstream of the front absorber during the LHC Run3 [264].

\subsection{JLAB: electron-photon discrimination at an electron beam facility}

The CLAS12 Forward Tagger (FT) calorimeter [265] was built to detect the electrons scattered at very small angles in the kinematics of quasi-real photo-production. This regime is of special interest for the search of exotic mesons and rare states, such as scalars and strange mesons [25]. The FT was designed for high efficiency electron-photon discrimination in this angular range, given the large number of photons produced in the target. It consists of an electromagnetic calorimeter (FT-Cal), to identify the electron in the energy range $0.5-4.5 \mathrm{GeV}$ and provide a fast trigger 
signal. A Micromegas tracker (FT-Trk) is used to measure the scattering angles and a scintillation counter (FT-Hodo) provides $\mathrm{e}-\gamma$ particle discrimination.

The FT-Cal design is driven by demanding requirements in terms of radiation hardness, compactness, energy and time resolution. To match these requirements, lead tungstate $\left(\mathrm{PbWO}_{4}\right)$ was used as scintillating material. The FT-Cal is composed of 332 crystals with dimensions $15 \times 15 \times 200 \mathrm{~mm}^{3}$, read out by avalanche photodiodes, with full azimuthal acceptance and forward angle $2^{\circ}<\theta<5^{\circ}$.

The discrimination between photons and electrons producing electromagnetic showers in the FT-Cal is performed via the FT-Hodo. This detector consists of 232 plastic scintillator tiles, read out by SIPMs, segmented in two layers to suppress the splash-back contribution from the FT-Cal.

The precise determination of the scattered electron angle is obtained with a tracker complementing the FT-Cal and FT-Hodo detectors. The FT-Trk uses the same technology adopted by the CLAS12 central and forward Micromegas tracker [266]; it consists of two double-layer detectors and is located in front of the FT-Hodo with geometry chosen to enable the determination of the $(x, y)$ coordinates (perpendicular to the beam $z$-axis) of a track.

The energy and centroid of the clusters in the FT-Cal are used as an initial seed to define the three-momentum of the incident particles. FT-Hodo hits are reconstructed and grouped into clusters, matching position and time in the tiles of the two layers of the detector. These are then matched to clusters in the calorimeter to distinguish charged particles from neutrals that do not give a signal in the FT-Hodo. Calibration and performance assessment were carried out through the analysis of data recorded with the CLAS12 production triggers [265]. Using the calibration constants obtained with the beam-on data, an energy resolution of $3.3 \%$ at $2.2 \mathrm{GeV}$ beam energy was extracted from the fit of the elastic peak in the e $\mathrm{p} \rightarrow$ e p process (see Fig. 34a). With the same calibration constants, the $\pi^{0} \rightarrow \gamma \gamma$ decay was reconstructed at $10.6 \mathrm{GeV}$ beam energy selecting events with both photons detected in the FT-Cal, finding a resolution of $4.4 \mathrm{MeV}$, corresponding to $3.2 \%$ (see Fig. 34b).

The calibration of the FT-Hodo was performed by studying the energy deposition of MIPs, being these the typical signals expected from charged particles impinging on the detector. The time information is critical for background rejection and for fasttrigger purposes. The study of the timing resolutions of the FT-Cal and FT-Hodo was performed by analyzing the time correlation of signals in the FT with the CLAS12 forward time-of-flight (FTOF) detector [267] and the RF signal that is synchronous with the CEBAF accelerator beam bunches. A resolution of $\sigma \simeq 140$ ps was obtained for electrons using the beam RF signal. A consistent value of $\sigma \simeq 150$ ps was found for photons, determining the event start time from other particles detected in the FTOF.

Since photons usually do not interact in the FT-Trk and FT-Hodo, the combined information of presence of clusters, position and timing of the sub-detectors allow to achieve a high photon-electron discrimination efficiency: the probability of a photon being erroneously identified as an electron has been estimated to be $\sim 4 \%$ using the FT-Cal and FT-Hodo combined information and $<1 \%$ with the contribution of the FT-Trk. 

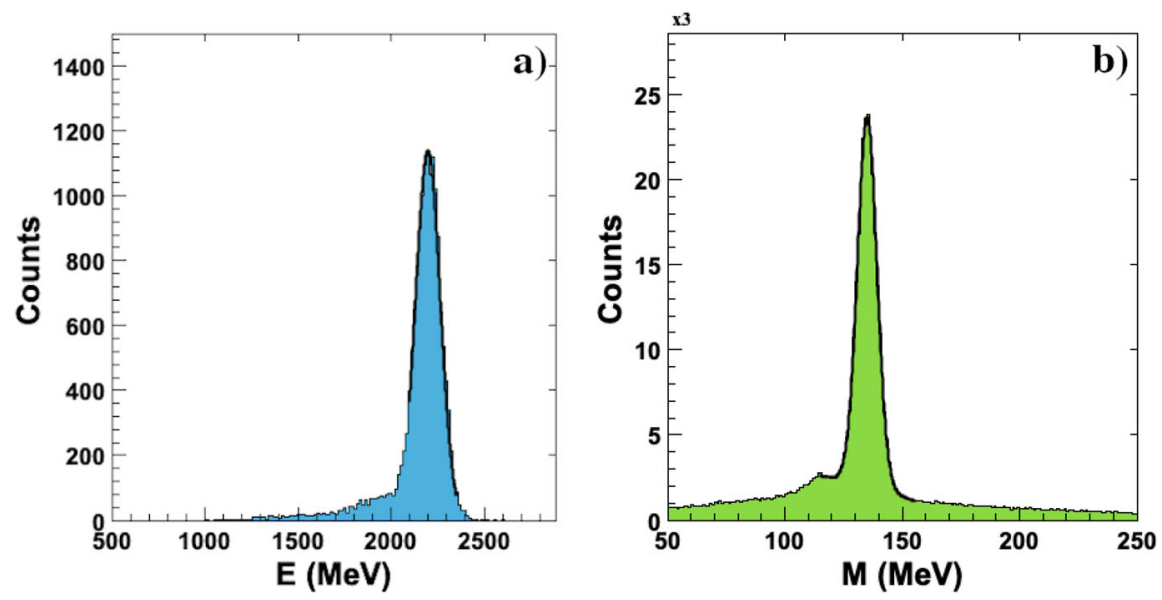

Fig. 34 JLAB. a Electron energy spectrum reconstructed in the FT-Cal at $2.2 \mathrm{GeV}$ beam energy. $\mathbf{b} \pi_{0} \rightarrow \gamma \gamma$ invariant mass spectrum reconstructed at $10.6 \mathrm{GeV}$ beam energy: the $\pi^{0}$ peak is fitted with a Gaussian with $\sigma \sim 4.4 \mathrm{MeV}$. From [265]

\subsection{BGOOD (MAMBO): neutral particles discrimination with BGO}

The BGO calorimeter of the BGOOD experiment is segmented into 480 crystals 24 cm-thick ( $\simeq 21$ radiation lengths), arranged in $15 \times 32$ sectors in the polar and azimuthal angles, respectively. The coverage on the polar angle is $\theta=\left(25^{\circ} \div\right.$ $155^{\circ}$ ) and full in azimuth. The calorimeter is preceded by the cylindrical scintillator barrel, which is used as a $\mathrm{d} E / \mathrm{d} x$ detector for charged particles and its granularity in the azimuthal angle is the same of the BGO sectors. Finally, closest to the target, two cylindrical multi-wire proportional chambers (MWPCs) allow charged particle tracking.

A first fundamental discrimination neutral/charged particles in the central detector is provided by the geometrical coincidence between the BGO sectors, the plastic scintillators of the barrel and the MWPCs. Signals in the BGO which are not associated with hits in any other detector are considered as interactions of neutral particles (photons or neutrons). We define a "cluster" as the group of contiguous crystals associated with the interaction of the same particle with the detector and its "multiplicity" is the number of crystals composing the cluster.

For photon/neutron discrimination, the different processes underlying the detection of the two types of particles in the BGO calorimeter must be considered: in the energy range explored $\left(E_{\gamma} \simeq 20-300 \mathrm{MeV}\right)$ while photons produce an electromagnetic shower in the high density, high atomic number inorganic material of the BGO, neutrons are mainly detected via their elastic and inelastic scattering (or even induced fission) on the different components of the BGO (oxygen, germanium, bismuth).

The main features of the clusters produced by high-energy photons or by neutrons are different. Cluster multiplicity is: (1) roughly proportional to the energy of the detected photon; (2) small and independent of the released energy for the neutron. To define further PID criteria, a clean sample of $\pi^{0}$ photoproduction events on deuterons 


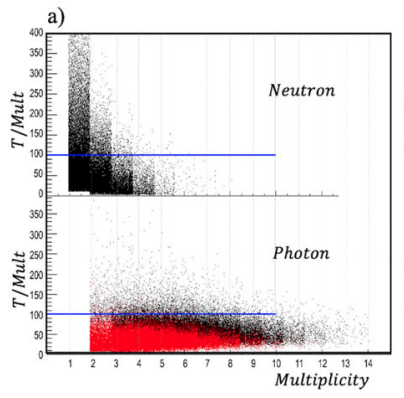

b)

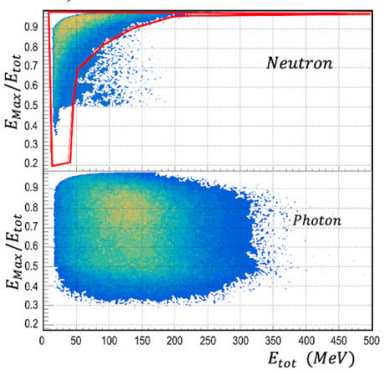

c)

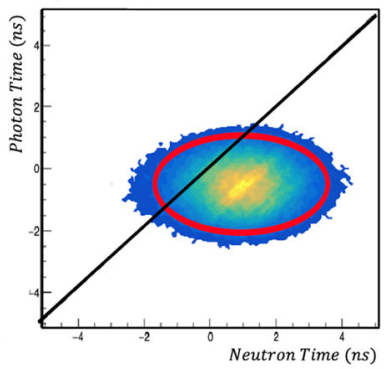

Fig. 35 MAMBO. a Ratio between the total cluster energy and the cluster multiplicity for neutrons and photons (in black and red the more and less energetic $\pi^{0}$ decay photons, respectively). b Ratio between the maximum energy released in a crystal and the total cluster energy for neutrons with multiplicity $>2$ and photons (sum of red and black distributions from $\mathbf{a}$ ). The applied graphical cut is the red contour. $\mathbf{c}$ photon TOF versus neutron TOF distribution for selected events, the applied cut is presented in red. Ambiguity between two neutral signals with the same multiplicity is solved assigning the neutron tag to the signal with larger TOF (see $45^{\circ}$ black line in the picture)

has been selected by means of stringent kinematic cuts on the 2-body reaction. The following PID criteria have been derived:

(1) the ratio between the total cluster energy and the cluster multiplicity is always $<100 \mathrm{MeV} /$ crystal for photons, while for neutrons also hits with larger amount of energy per crystal are observed (see Fig. 35a);

(2) the ratio between the maximum energy released in a crystal of a cluster and the total cluster energy has a different distribution for neutrons and photons with energies $>25 \mathrm{MeV}$. This allows to apply a graphical cut on the neutron distribution for neutrons with multiplicity $>2$ (see Fig. 35b);

(3) the TOF (defined with respect to an arbitrary $t_{0}$ ) is in average higher for neutrons ( $\simeq 1 \mathrm{~ns}$ ) than for photons $(\simeq-0.5 \mathrm{~ns}$ ) (see bisector in Fig. $35 \mathrm{c})$. This allows to apply an ellipsoidal cut (in red in Fig. 35c) to suppress the background due to low-energy photons and out-of-time events.

The time measurement also allows one to solve the ambiguity between low-energy neutral clusters with the same multiplicity, since the cluster with the larger time value is associated with the neutron. Applying the previously described selection criteria to the same sample of events before the kinematical cuts, the sample is reduced of $\sim 40 \%$. In further detail, for a global reduction of $40 \%$ of the sample, cut (1) causes a reduction of $\sim 5 \%$, cut (2) of $\sim 10 \%$ and cut (3) contributes with an additional $25 \%$.

\section{Ring-imaging Cherenkov detectors}

The discovery of the emission of photons by a charged particle travelling in a material at a speed greater than the in medium speed of light (the Cherenkov effect) opened the possibility to design a full class of detectors. The principle can be implemented in several ways. The use of the bare emission of light above a velocity threshold proved to be very effective even in very narrow phase space, the so-called differential Cherenkov detector, as it turned out for example in the experiment which discovered 
the antiproton [268]. Arthur Roberts proposed in 1960 the Cherenkov light-ring imaging "for the accurate measurement of particle velocity and direction" exploiting the directional properties of Cherenkov light. The seminal work of Seguinot and Ypsilantis [269] demonstrated the applicability to large area detectors. The early 80's saw a first generation of devices, which suffered of performance problems, while the second generation, in the late $80 \mathrm{~s}$, worked well enough for the experiments, even with a reduced quantum efficiency of the converters. The evolution of the techniques to detect photons (from the first photon-sensitive vapors coupled to multiwire chambers, to photomultipliers, to modern silicon photomultipliers) has been critical for the feasibility of devices covering large fractions of the phase space, eventually a key interest point. The dependence of the momentum range of particles identifiable with this technique represented an opportunity to better adapt the detectors to the experimental conditions and, where it could represent a limit for the wide momentum range to explore, it triggered ingenious optical solutions and the development of new radiators materials. RICH counters are always complementary to the other PID techniques, extending the energy range of the particle identification. In this section few applications in CSN3 experiments are described, emphasizing the different technical solutions and demonstrating the versatility of the technique.

In the ALICE and CLAS12 experiments, although the energy range of detected particles is similar and below $10 \mathrm{GeV} / c$, the $\mathrm{RICH}$ detectors differ in the radiators and in the design of the optical paths: at ALICE $\mathrm{C}_{6} \mathrm{~F}_{14}$ is used as radiator together with a proximity focus (no optics), providing also a position measurement of the charged particles on the cathodes, while at CLAS12 aerogel was chosen as radiator and geometrical constraints were matched thanks to a mixed spherical/plane mirrors solution.

The EIC experiment, whose first measurement is foreseen in 2030, is considering various options for their RICH detector with the aim to extend the particle separation up to $60 \mathrm{GeV} / c$. It is an opportunity to explore the most recent technical advances, on the front of the radiators (with an eco-friendly possibility), of the design which demands for compactness, and of single-photon detectors like SiPM's or micro-pattern gaseous detectors.

\subsection{ALICE: the largest CsI RICH application for pion, kaon, proton separation}

The ALICE HMPID (High-Momentum Particle IDentification) detector [270] represents the largest-scale application of CsI photocathodes, setting the standard for many detectors using the same technology, and is the largest device of its kind operated at a collider. It consists of seven proximity-focusing RICH counters and exploits MWPCs, coupled with a pad segmented CsI coated photo-cathode, to detect both Cherenkov photons and charged particles. With an active area of $10.3 \mathrm{~m}^{2}$, it covers $\approx 5 \%$ of the ALICE central barrel acceptance in the pseudo-rapidity range $|\eta|<0.5$. The information of the pads is used to define clusters whose centroid is used as the reconstructed position of the photon. The HMPID identifies charged hadrons combining the measurement of the emission angle of the Cherenkov photons produced in a 1.5cm-thick layer of low chromaticity $\mathrm{C}_{6} \mathrm{~F}_{14}$ (perfluorohexane) liquid radiator with an 


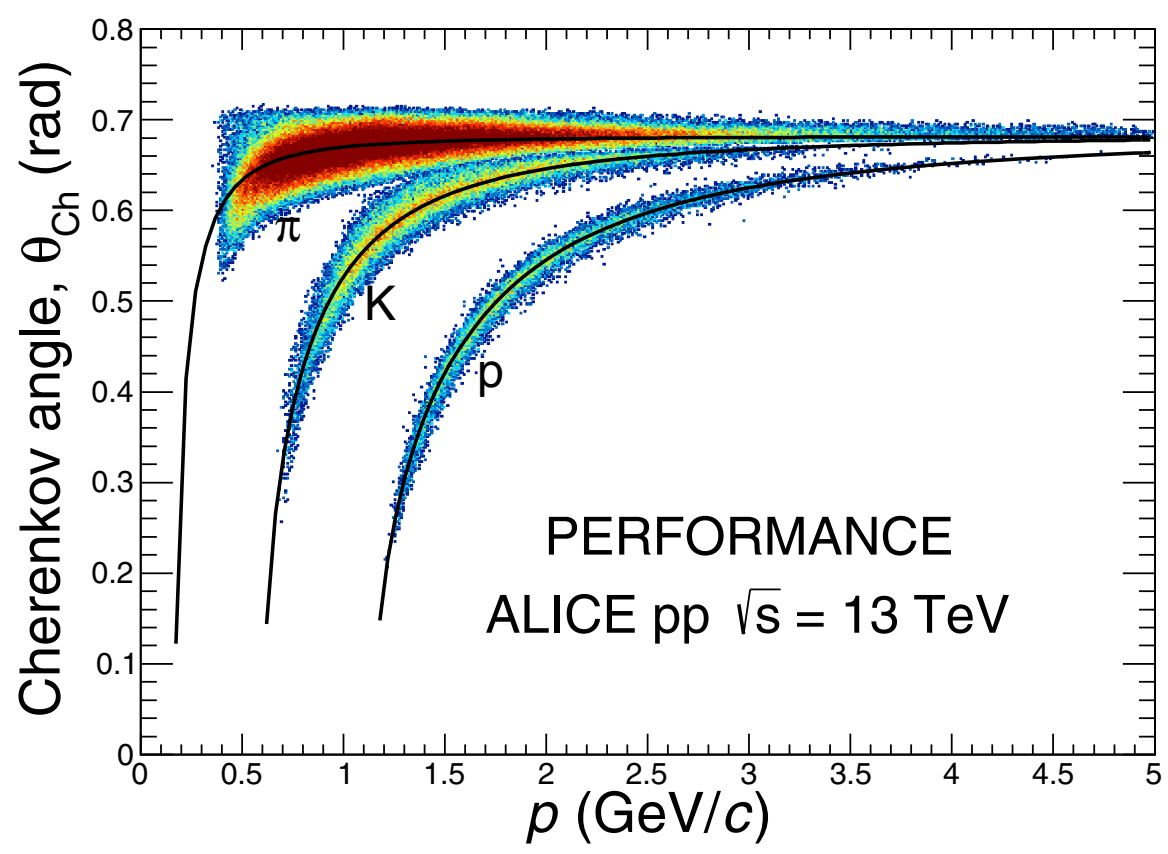

Fig. 36 ALICE. a Cherenkov angle measured in the HMPID vs track momentum. b The separation in units of $\sigma$ for $\pi / \mathrm{K}$ and $\mathrm{K} / \mathrm{p}$ as a function of transverse momentum in the HMPID, from [272]

index of refraction of $n=1.2989$, with the momentum information provided by the main ALICE tracking detectors, the ITS and the TPC (see Sect. 3.3). This requires the track extrapolation from the central tracking detectors to the MIPs reconstructed position on the HMPID photo-cathode. Starting from this coordinates, candidate photon interaction points are selected around the MIP and the photon emission angle is calculated. This procedure is called backtracing [270]. To associate the Cherenkov emission angle, deduced from the photon ring reconstruction, to the track and reject the background contribution, the Hough Transform Method (HTM) [271] is applied. Figure 36, left panel, shows the scatter plot of the measured Cherenkov angle as a function of the track momentum for pp collisions at $\sqrt{s}=13 \mathrm{TeV}$ collected in 2015. The three bands of pions, kaons and (anti-)protons are clearly visible and centered on the theoretical values (black curves).

The particle yields are then extracted from a three-Gaussian fit to the Cherenkov angle distribution in narrow transverse momentum bins. The multiplicity of $\approx 90$ primary tracks plus secondaries produced in the most central $(0-5 \%) \mathrm{Pb}-\mathrm{Pb}$ collisions in the HMPID acceptance corresponds to an average detector occupancy of $\approx 3.5 \%$. In these conditions the probability that HTM detects fake Cherenkov patterns increases. The larger the emission angle, the larger the probability to find background clusters while scanning for Cherenkov photons. The background on the photo-cathodes is uniform and for a larger emission angle a larger area is scanned, so background clusters can reproduce Cherenkov patterns. To remove the background a polynomial function of 6th order is used to fit the distribution of the identified fake patterns. 
Figure 36, right panel, shows the separation in units of $\sigma$ for $\pi / \mathrm{K}$ and $\mathrm{K} / \mathrm{p}$ as a function of transverse momentum in the HMPID, for pp collisions at $\sqrt{s}=13 \mathrm{TeV}$ and $\mathrm{Pb}-\mathrm{Pb}$ collisions at $\sqrt{s_{\mathrm{NN}}}=5.02 \mathrm{TeV}$, respectively. The precision of the fitting of the background distribution represents a source of systematic uncertainty. In pp and $\mathrm{p}-\mathrm{Pb}$ collisions, where the background is negligible and the background fitting is not applied, the total systematic error is smaller. Exploiting the statistical unfolding, the HMPID has provided pion and kaon $p_{\mathrm{T}}$ spectra between $1.5 \mathrm{GeV} / c$ and $4 \mathrm{GeV} / c$ and (anti-)proton spectra in the $p_{\mathrm{T}}$ interval $1.5-6 \mathrm{GeV} / c$. After ten years from the first operation, the HMPID performance is stable and the PID capabilities are at the level of expectations.

\subsection{EIC: perspective of PID techniques at the electron-ion collider}

Different PID technologies are proposed at EIC to identify hadrons over a wide kinematic range and to reinforce electron/hadron separation where electromagnetic calorimetry is the main player.

For hadron PID at high momenta, there are no alternatives to a gaseous RICH. The INFN groups are actively contributing to the development of the modular RICH (mRICH) for low momenta PID, in the R\&D activities toward a dual RICH (dRICH) and in developing gaseous detectors of single photons based on MicroPattern Gaseous Detector (MPGD) technologies, to be used in a windowless RICH. An approach to eco-friendly gaseous RICHes has also been proposed.

The mRICH aims to provide $\pi-\mathrm{K}$ separation in the low-momentum range (3$10 \mathrm{GeV} / c$ ) and e- $\pi$ separation up to $2 \mathrm{GeV} / c$ [273-276]. It is an aerogel $\mathrm{RICH}$, where the simple proximity focusing scheme is enhanced using Fresnel lenses with the goal of raising the upper limit of the effective momentum range, keeping a mandatory compact design.

The need for an extended momentum range can be fulfilled exploiting the availability of high clarity, hydrophobic aerogel ${ }^{7}$ radiators, which has driven the proposal of the dRICH $[277,278]$. The dRICH foresees $\mathrm{C}_{2} \mathrm{~F}_{6}$ as gas radiator $(n \simeq 1.0008)$ and an aerogel radiator $(n \simeq 1.02$ ) for $3 \sigma \pi / K$ separation in the range $3-60 \mathrm{GeV} / c$ and efficient $\mathrm{e} / \pi$ separation from few hundred $\mathrm{MeV}$ up to $\sim 15 \mathrm{GeV} / c$ in the EIC spectrometer forward region. The design has been supported with a complete Geant4 [279] simulation study and extensively using AI techniques with the Bayesian optimization to determine the optimal design parameters [280]. Figure 37 shows a typical simulated event in the dual RICH. A first prototype has been designed and is presently under construction, in view of a test beam exercise in 2021. The readout of the Cherenkov light is another field of development: the well deployed MultiAnode PMTs (MAPMT) which are being used to test the initial RICH prototypes, are not suitable for the setup at EIC because they should operate in a strong magnetic field. SiPMs as photon sensors make an appealing alternative, even if more tests are needed to establish the procedures to keep under control the noise rate, especially after irradiation. Dedicated studies are

\footnotetext{
7 The aerogel is an amorphous solid network of $\mathrm{SiO}_{2}$ nanospheres. The transmission $T$ of light of wavelength $\lambda$ through a material of length $L$ can be parameterised as $T=T_{0} \exp -\frac{C \cdot L}{\lambda^{4}}$ where $T_{0}$ describes the bulk properties of the aerogel and $C$, the clarity, the wavelength dependence.
} 


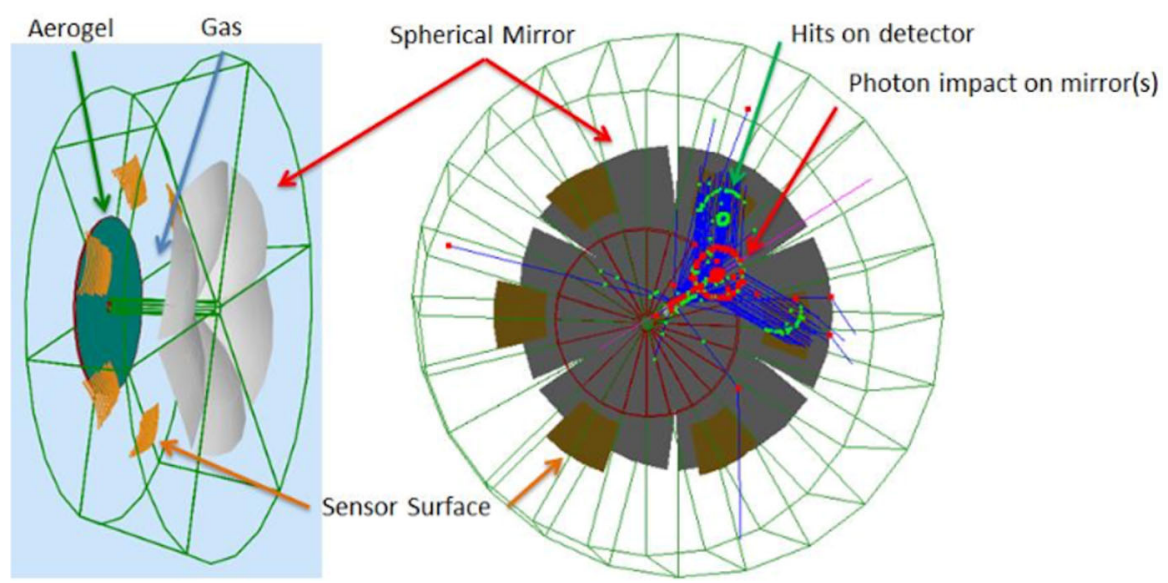

Fig. 37 EIC. Dual RICH detector configuration after AI-driven optimization. Multiple mirror panels (gray) focus rings from both aerogel and $\mathrm{C}_{2} \mathrm{~F}_{6}$ per-fluorocarbon radiators onto the same focal plane (GEANT4 simulation). Adapted from [280]

being performed to reach the goal of SiPM that can operate up to an integrated fluxes of $10^{11} 1-\mathrm{MeV}$ neutron equivalent per $\mathrm{cm}^{2}$ [281], to ensure several years of operation at maximum EIC luminosity, along with the coupling to the front-end ALCOR chip [282], developed for the SiPMs of the DarkSide experiment. A possible direction to explore comes from recent evidence [283] that the target radiation hardness is reachable when operating SiPMs at low temperature (in the range -30 to $-40{ }^{\circ} \mathrm{C}$ ) and undertaking annealing cycles at high temperature $\left(170^{\circ} \mathrm{C}\right)$. An initial exercise of $\mathrm{mRICH}$ read-out using SiPMs as photon sensors has been performed. To take advantage of the higher density of Cherenkov photons in the far UV (the distribution depending on $1 / \lambda^{2}$ ) and to cope with compactness requirements, the windowless design has been introduced [284], which avoids light absorption, using gaseous detectors like the PHENIX HBD [285].

Gaseous detectors are established cost effective solutions to cover large-area single photon detection surface in magnetic field with low material budget. So far, CsI is the only available photo-cathode (PC) in the far UV domain with high quantum efficiency (QE). MPGD based photon detectors (PDs) demonstrated natural answer to control ion backflow (IBF) and photon feedback: few examples are the COMPASS RICH upgrade [286,287], the windowless RICH prototype and test beam with quintuple GEM PDs [284], the TPC-Cherenkov (TPCC) tracker prototype with quadruple GEM PDs [288].

In the EIC context a multi-directional R \& D program is led by INFN:

- establishment of the hybrid PD for a windowless RICH approach to increase the number of detected photons;

- increasing the granularity of the readout elements for fine resolution with limited lever arm. Figure 38 shows a schematic drawing of the COMPASS-like photon detector in the 2018 beam test and an example of the detected Cherenkov ring [289];

- comparison the detector performance using either THGEM (as in COMPASS) or GEMs for the first multiplication stages; 

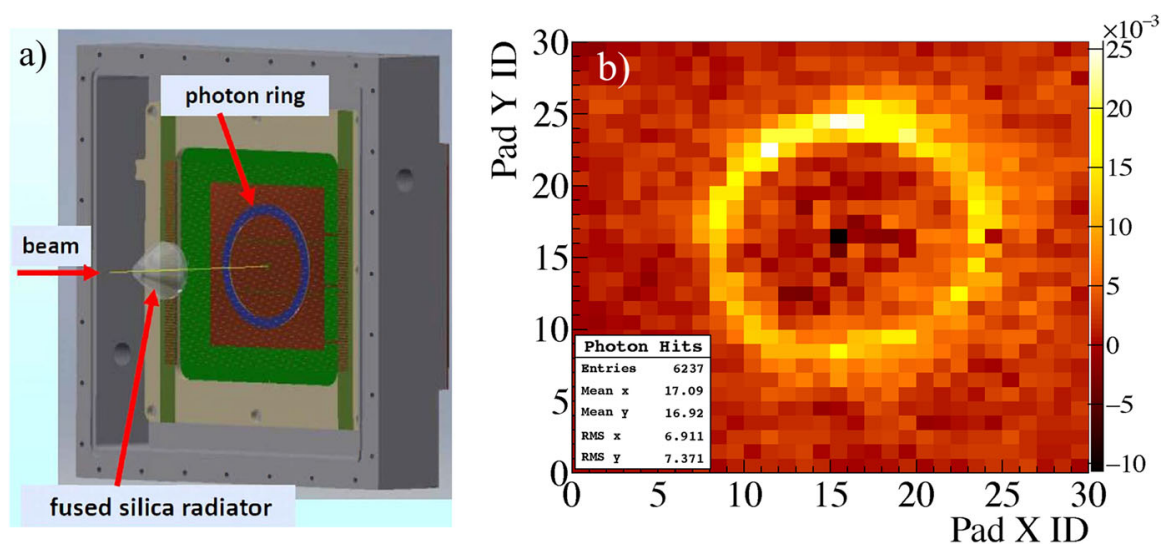

Fig. 38 EIC. a The formation of the ring image on the photon detector prototype by Cherenkov photons generated in a quartz radiator crossed by beam particles. b 2-D histogram of the hits produced by the Cherenkov photons in the small pad-size prototype

- identification of an adequate front-end chip: studies for coupling the hybrid PD with VMM3 ASIC have been initiated;

- coupling of the THGEMs with a novel and more robust PCs by Hydrogenated Nano Diamond powder (HND) to overcome the limitation imposed by the use of CsI [290]. Set of systematic studies are ongoing for complete validation [291,292].

Due to the Global Warming Power of fluorocarbons, their application is expected to be more and more limited. For this reason, pressurised Ar at a few bar level has been proposed as alternative [293]. Initial performance studies have started [294], while the mechanical challenge posed by the need of a light-material vessel standing the pressure is still to be addressed.

\subsection{JLAB: advanced compact and optimised design of RICH at CEBAF}

The baseline detectors composing the CLAS12 spectrometer (time-of-flight and threshold gas Cherenkov counters) are able to efficiently identify electrons, and separate hadron species at momenta below $3 \mathrm{GeV} / c$. The realization of a different kind of detector, based on Cherenkov ring imaging technique (RICH), has been led by INFN to properly identify particles at higher momenta up to $8 \mathrm{GeV} / c$. The design of the CLAS12 RICH posed many challenges. It had to fit inside the CLAS12 spectrometer, imposing geometrical and material budget constraints. The momentum range of interest suggested the use of aerogel (see note 7) as radiator, with refraction index in between gases and liquids $(n=1.05)$, with an unprecedented clarity [295]. A unique hybridoptic solution, with photons at different angles undergoing different path lengths, has been adopted using plane and spherical mirrors [296,297]. The planar mirrors exploit for the first time a $0.7 \mathrm{~mm}$ glass-skin technology, developed for the terrestrial telescopes, to obtain a material budget comparable to carbon fiber at a much reduced cost [298]. The CLAS12 RICH works in the visible and near-UV wavelength range to avoid the more and more evident Rayleigh scattering inside aerogel in the UV region, and it is the first large scale device using flat-panel large area multi-anode photo- 


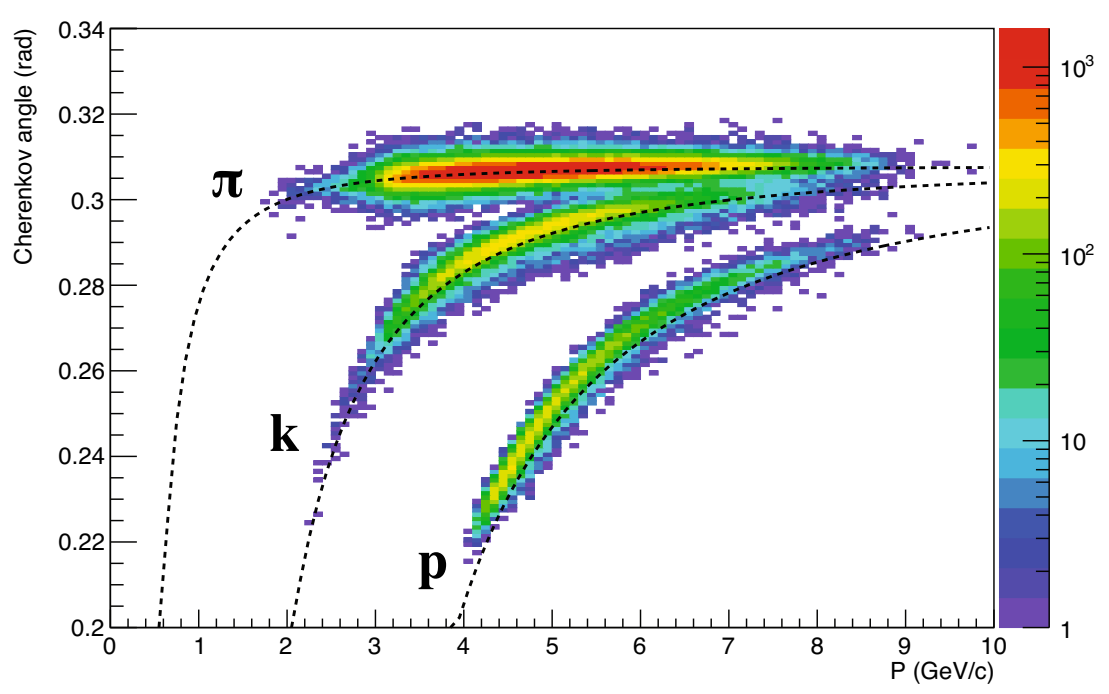

Fig. 39 JLAB. RICH reconstructed mean Cherenkov angle as a function of the particle momentum for positive hadron tracks, impinging on aerogel tile 13 in layer 0. Pions, kaons and protons are separated over the full 3 to $8 \mathrm{GeV} / c$ momentum range. The small deviations with respect to the expected pattern (dashed lines) for the three particle hypotheses are due to the not yet optimized alignment of the RICH components

multipliers (MAPMTs). The RICH front-end electronics, counting 25,000 channels, ensures $100 \%$ efficiency at 1/3 of a photoelectron level, 1:4 gain spread compensation, and time resolution of the order of $1 \mathrm{~ns}$ to distinguish direct from reflected photon hits, while accepting trigger rates up to $20 \mathrm{kHz}$ with a latency of $8 \mu \mathrm{s}$.

The first RICH sector was installed on January 2018, in time for the start of the CLAS12 data-taking [299].

Although the alignment of the various sub-components and the fine calibration of the Cherenkov angle response is still ongoing, the preliminary results show that the detector matches the required performance in discriminating between pions and kaons over the full range between 3 and $8 \mathrm{GeV} / c$, see Fig. 39. For a single detected photon, the measured time resolution is better than $1 \mathrm{~ns}$ and the angular resolution is better than $5 \mathrm{mrad}$. In the direct configuration (expected to be largely unaffected by the ongoing sub-component alignment), the complete ring reconstruction meets a $1.5 \mathrm{mrad}$ resolution per track, corresponding to the design $4 \sigma$ pion-kaon Cherenkov angle separation at the highest $8 \mathrm{GeV} / c$ momentum. A second $\mathrm{RICH}$ sector is under construction and expected to be installed in late 2021 .

\section{Kinematic and Bayesian methods}

Measurement of the yields of rare nuclear processes can be done using kinematic methods. Examples of these are the THM (Trojan Horse Method) [98] largely used by the ASFIN experiment (see Sect. 2.4.1) and the kinematical constraints used by the CHIRONE (see Sect. 2.3.1) and NUCLEX (see Sect. 2.3.4) experiments.

The Bayesian approach is a method to combine the PID signals obtained from different detectors of the same experiment by folding the probabilities with the expected 
abundances of each particle species. ALICE experiment (see Sect. 2.2.1) has used this approach in many analyses [300,301], showing the large improvement obtained in particle identification [302].

\subsection{Kinematic method}

\subsubsection{ASFIN: identification of undetected particles through momentum conservation}

The Trojan Horse Method (THM) [99] allows to study the nuclear reaction with three bodies in the final state by detecting and identifying only two of the three particles and measuring their kinematic quantities (angle of emission and energy). Indeed, the experiment can be considered kinematically complete, since all kinematic variables can be derived from the measured quantities.

This procedure cannot be used when the reaction takes place on unknown target nuclei, e.g. on target contamination. Let us consider the reaction $P+T \rightarrow a+b+s$, where $P$ is the projectile nucleus and $T$ is the target nucleus, $a, b$ and $s$ the produced particles. In case of target contamination, detecting only $a$ and $b$ particles implies that the system can be left undetermined due to the overlapping of different kinematic loci in the same phase-space region, corresponding to reactions having different undetected particles. Therefore, identification of particle $s$ is pivotal to the following data analysis, to reject possible background events.

A useful procedure that allows the determination of the mass number of the undetected particle $s$ and also of the $Q$-value spectrum of the reaction of interest consists in the introduction of two new variables [303]:

$$
\begin{aligned}
X & =\frac{p_{s}^{2}}{2 u} \\
Y & =E_{P}-E_{a}-E_{b},
\end{aligned}
$$

where $E_{P}, E_{a}$ and $E_{b}$ are the kinetic energies of projectile $P$ and particle $a$ and $b$; and $p_{s}$ is the momentum of $s, u \sim 931.5 \mathrm{MeV} / c^{2}$ is the atomic mass unit. The momentum $p_{s}$ is deduced from the energies and emission angles of $a$ and $b$ particles by applying the momentum conservation equation, thus the mass unit is not included in the variable $X$. The quantities $\mathrm{X}$ and $\mathrm{Y}$ are related through the relation:

$$
Y=\frac{1}{A_{s}} X-Q
$$

since $p_{s}^{2}=2 E_{s} A_{s} u$, with $p_{s}, E_{s}$ and $A_{s}$ momentum, kinetic energy and mass in units of $u$ of the $s$ particle and $Q$ the $Q$-value of the reaction. At this point, $A_{s}$ and $Q$ are unknown quantities and if experimental points corresponding to the measured coincidence events are reported on the $X-Y$ plane, one expects them to fall around a straight line, defined by Eq. 7. This line intercepts the $Y$-axis at $Y=-Q$ and has a slope given by $1 / A_{s}$, hence the values of $Q$ and $A_{s}$ can now be deduced from a fit to the data.

This so-called energy-momentum plot (EP-plot) is widely used to cross check the right channel selection in experiments which use THM. For example, in Fig. 40 it 
Fig. 40 ASFIN.

Energy-momentum plot for $\alpha-{ }^{16} \mathrm{O}$ coincidences as reported in [304]. The line is calculated assuming $A_{s}=1$ and a theoretical $Q$-value of $5.889 \mathrm{MeV}$. Events not following the line of unitary mass are discarded in the further analysis. Figure adapted from [304]

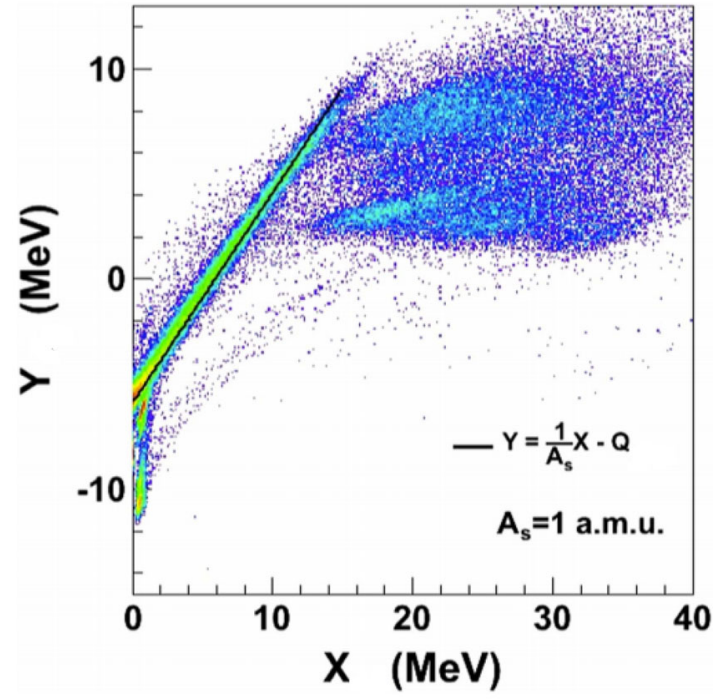

is reported the kinematic technique described above for selecting the ${ }^{19} \mathrm{~F}\left(p, \alpha_{0}\right){ }^{16} \mathrm{O}$ reaction channel, i.e. with ${ }^{16} \mathrm{O}$ in the ground state, from the measured three-body ${ }^{2} \mathrm{H}\left({ }^{19} \mathrm{~F}, \alpha{ }^{16} \mathrm{O}\right) \mathrm{n}$ reaction [304]. In this figure, the events corresponding to the channel of interest gather around the straight line of equation $Y=X-5.889$, as expected if a particle of $\mathrm{A}=1$ unit mass is emitted in a reaction with a $\mathrm{Q}$-value of $5.889 \mathrm{MeV}$.

\subsubsection{CHIRONE: background rejection through kinematic coincidences}

For a $4 \pi$ multidetector like CHIMERA the measurement of kinematical coincidences, in binary or complex reactions, is possible thanks to the complete angular coverage of the detector. The CHIRONE collaboration developed various techniques, based on imposing kinematical constraints, useful to improve the results of data analysis [218,219]. A typical improvement is the extraction of precise angular distributions of emitted particles [305].

Even more important is the cleaning effect on random coincidences obtained using kinematic constraints on momentum and energy conservation. This is a very powerful method that allows to measure very low probability channels with a good signal over background ratio. A relevant example was recently obtained in the measurement of the $\gamma$-ray decay channel of the Hoyle and of the $9.64 \mathrm{MeV}$ excited levels of ${ }^{12} \mathrm{C}$. Such levels are very important for the carbon production in astrophysical environments. Their decay probability is very low, $10^{-4}$ for the Hoyle state [306], while it is of the order of $10^{-6}$ for the $9.64 \mathrm{MeV}$ level, see Ref. [219]. The CHIRONE collaboration was able to perform such measurement using an $\alpha$ beam, accelerated at $64 \mathrm{MeV}$, and a target of ${ }^{12} \mathrm{C}$. The background was strongly suppressed, with the request of momentum and energy conservation applied to all detected particles and $\gamma$-rays. In the case of this experiment, this implies a quadruple coincidence of two charged particles ( $\alpha$ and ${ }^{12} \mathrm{C}$, identified by means of $\Delta \mathrm{E}-\mathrm{E}$ (see Sect. 3.2.1) and $\Delta \mathrm{E}-\mathrm{TOF}$ (see Sect. 6.2.2), respectively) and two $\gamma$-rays. 

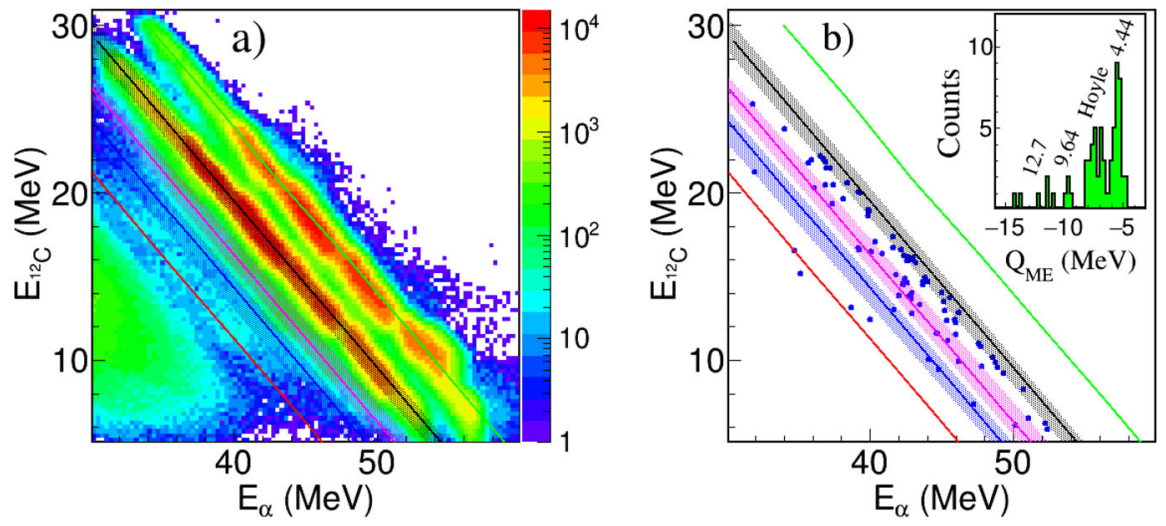

Fig. 41 CHIRONE. a Kinematic loci obtained plotting the energy of recoiling ${ }^{12} \mathrm{C}$ against the energy of scattered $\alpha$ particle, in the $\alpha+{ }^{12} \mathrm{C}$ scattering at $64 \mathrm{MeV}$. The lines and regions of interest are the expected kinematics loci for the elastic scattering (green), 4.44 MeV level (black), 7.65 MeV Hoyle state (purple), 9.64 MeV (blue) and $12.7 \mathrm{MeV}$ level (red). b As in a in coincidence with two $\gamma$-rays. The upper inset shows the $\mathrm{Q}_{\mathrm{ME}}$ obtained for the same events (figure adapted from Ref. [219])

Figure 41a shows the kinematic loci obtained plotting the energy of the ${ }^{12} \mathrm{C}(\mathrm{y}-$ axis) and the energy of the scattered $\alpha$ particle (x-axis). Lines and regions of interest with different colors show the expected kinematic loci for different levels of ${ }^{12} \mathrm{C}$. In this case, only the two kinematic loci of elastic scattering and $4.44 \mathrm{MeV}$ level are evidently populated; events of the $12.7 \mathrm{MeV}$ level are also present, while the other levels are overwhelmed by the background. On the contrary, Fig. 41b shows the same correlation after the kinematics coincidence events with two $\gamma$-rays. In this figure, the contribution of elastic scattering is completely suppressed while the contribution produced by the tail of the $4.44 \mathrm{MeV}$ level, due to spurious coincidences, emerges. This result is visible also in the inset of Fig. $41 \mathrm{~b}$ showing the $Q_{\mathrm{ME}}$ spectrum obtained for the same events of Fig. 41b. The $Q_{\mathrm{ME}}$ is given by the difference between the total detected kinetic energy and the available beam energy: $Q_{\mathrm{ME}}=\operatorname{Energy}\left(\alpha_{\text {scattered }}\right)+\operatorname{Energy}\left({ }^{12} \mathrm{C}\right)$ $\operatorname{Energy}\left(\alpha_{\text {beam }}\right)$. With this method a background rejection factor of the order of $\approx 10^{5}$, that allows to extract information about the Hoyle state, is obtained [219].

\subsubsection{NUCLEX: correlation analysis for rare event selection}

In nuclear reactions, several isotopes are produced in their internal unbound states. These states decay into two or more particles and precise measurements of their momentum vectors allow to reconstruct the invariant mass of their original decaying parent isotope that can, therefore, be identified experimentally. This can be accomplished with OSCAR, FAZIA and GARFIELD multi-detector arrays (see Sect. 3.2.2 for details). The energy position and the shape of the observed resonance peaks carry information also about structure properties of the unbound isotope. Within this context, the NUCLEX collaboration has studied the three $\alpha$ decay of the Hoyle state at $\mathrm{E}_{X}=7.654 \mathrm{MeV}$ in ${ }^{12} \mathrm{C}$, playing a key role in cluster structure and in nuclear astrophysics. Special attention has been devoted to the branching ratios of a direct three- $\alpha$ decay (DD) vs. a sequential decay path passing through the production of ${ }^{8} \mathrm{Be}$, namely 


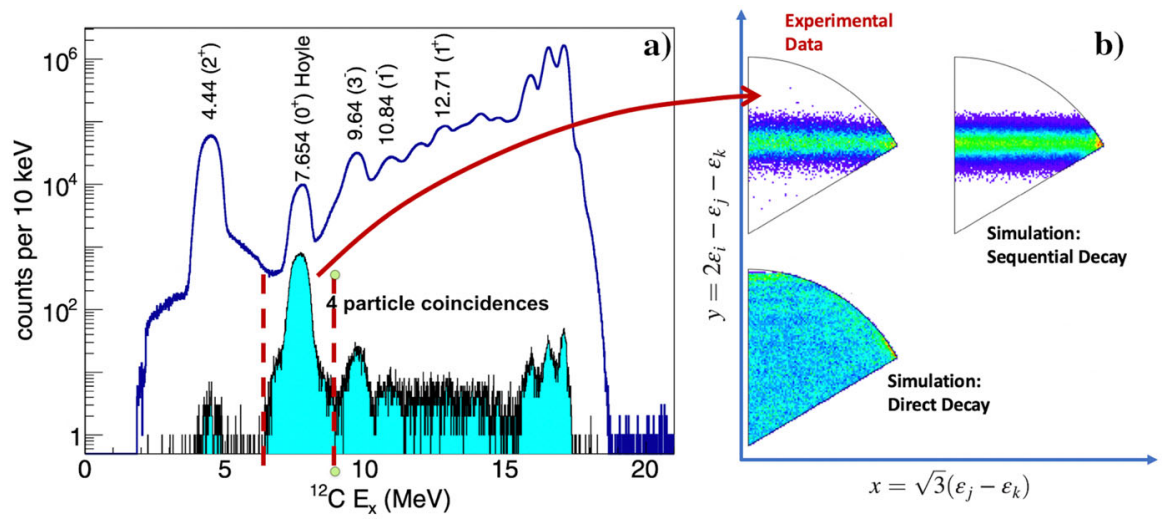

Fig. 42 NUCLEX. a ${ }^{12} \mathrm{C}$ excitation energy spectrum reconstructed with three- and four- $\alpha$ particle coincidences in ${ }^{14} \mathrm{~N}(\mathrm{~d}, \alpha){ }^{12} \mathrm{C}$ at $E=10.5 \mathrm{MeV}$. b Dalitz plot analyses of the three alpha coincidences produced by the Hoyle state. From [307]

${ }^{12} \mathrm{C} \rightarrow \alpha+{ }^{8} \mathrm{Be}$ followed by ${ }^{8} \mathrm{Be} \rightarrow \alpha+\alpha$. In Ref. [307], the Hoyle state was produced with the transfer reaction ${ }^{14} \mathrm{~N}(\mathrm{~d}, \alpha){ }^{12} \mathrm{C}^{\text {Hoyle }}$, studied with deuteron beams at $\mathrm{E}=10.5 \mathrm{MeV}$ accelerated at the LNS, using a hodoscope designed by the NUCLEX groups and constituting also the OSCAR detector project. A $\triangle \mathrm{E}$-E Silicon telescope was used to detect the $1+3 \alpha$ particles produced in the final state, thus deducing the residual excitation energy of the produced ${ }^{12} \mathrm{C}$ nucleus, $\mathrm{E}_{X}$, shown in Fig. 42a as a blue-line histogram. The observed resonance peaks correspond to ${ }^{12} \mathrm{C}$ internal states. By correlating the detected $\alpha$ particle with other three- $\alpha$ coincidences detected by a 64 Silicon pads hodoscope, it was possible to precisely identify the Hoyle state at $\mathrm{E}_{X}=7.654 \mathrm{MeV}$ with negligible background (see blue-filled histogram on Fig. 42). A Dalitz plot analysis of these three $\alpha$ coincidences is shown in Fig. 42b in the form of special two-dimensional Dalitz plots. The top-left Dalitz plot is constructed with the experimental events falling within the Hoyle state peak (see [307] for details on the analysis and variables used). The top-right and bottom-left two-dimensional Dalitz plots are built with a Monte Carlo simulation of the Hoyle state decay into three- $\alpha$ 's in sequential and direct mode, respectively. Using this kinematic approach, the comparison to experimental data clearly shows how the sequential mode fulfills the whole decay width of the Hoyle state. Similar kinematic multi- $\alpha$ coincidence studies have been performed with the GARFIELD array coupled to the the RCo ring counter at the LNL [308,309]. Similar methods are used also with the FAZIA detector in heavy-ion collisions, where a large number of decaying states can be isolated and explored.

\subsection{Bayesian methods}

\subsubsection{ALICE: combining PID techniques using a Bayesian approach}

In ALICE charged particles as $\pi^{ \pm}, \mathrm{K}^{ \pm}$, and $(\overline{\mathrm{p}}) \mathrm{p}$ are identified independently [310313] by the ITS (see Sect. 3.3), TPC (see Sect. 3.3), TOF (see Sect. 6.1.1) and HMPID (see Sect. 8.1) sub-detectors. However there is a large superposition among the trans- 
verse momentum $p_{\mathrm{T}}$ ranges of the ALICE subdetectors with a pion-kaon and kaonproton separation power equal or larger than $2 \sigma$ (see Figure 46 of [41]). The measured $p_{\mathrm{T}}$ spectra are usually combined in the overlapping ranges using a weighted average with the systematic and statistical uncertainties as weights. In this way it is possible to measure charged hadron $p_{\mathrm{T}}$ spectra from few hundreds of $\mathrm{MeV} / c$ up to $20 \mathrm{GeV} / c$

Starting from the detector signals it is possible to evaluate the probability in a specific detector for a given track to be one of the five charged particle species (electron, muon, pion, kaon, (anti-)proton). As an alternative to the previous described method, a Bayesian approach [300,301] to combine PID probabilities from different detectors was also used by ALICE.

The response of each detector can be expressed in terms of its raw signal $S$, e.g. the time of flight $t_{\text {TOF }}$ from the TOF detector, or the energy loss $\mathrm{d} E / \mathrm{d} x$ measured by the ITS or TPC, or the Cherenkov emission angle measured by the HMPID. $P\left(S_{\alpha} \mid H_{i}\right)$ is defined as the conditional probability that a particle of species $H_{i}$ will produce a signal $S_{\alpha}$ in a detector $\alpha$. The index $i$ can refer to electrons, pions, protons, etc. The combination of the probabilities from different detectors, $P_{\alpha}$, can thus be written as a product:

$$
P\left(\vec{S} \mid H_{i}\right)=\prod_{\alpha} P_{\alpha}\left(S_{\alpha} \mid H_{i}\right)
$$

where $\vec{S}=\left(S_{\mathrm{ITS}}, S_{\mathrm{TOF}}, \ldots\right) \cdot P\left(\vec{S} \mid H_{i}\right)$ is then the conditional probability that the set of detector signals $\vec{S}$ will be observed for a given particle species $H_{i}$.

However, the variable of interest in this case is the conditional probability $P\left(H_{i} \mid \vec{S}\right)$ that the detected particle is of the species $H_{i}$, given a certain signal $S$. The relation between the two conditional probabilities, for a combined set of detectors, can be expressed with Bayes theorem [314]:

$$
P\left(H_{i} \mid \vec{S}\right)=\frac{P\left(\vec{S} \mid H_{i}\right) C\left(H_{i}\right)}{\Sigma_{k=e, \pi, p, \ldots} P\left(\vec{S} \mid H_{k}\right) C\left(H_{k}\right)},
$$

where $C\left(H_{i}\right)$ is the a priori probability of measuring the particle species $H_{i}$ (i.e. the prior $)$, and the conditional probability $P\left(H_{i} \mid \vec{S}\right)$ is the posterior probability.

The choice of the prior represents the "best guess" one can make of an unknown quantity (in this case, the true particle yields per event). The subjectivity of the priors choice is then eliminated by means of an iterative procedure described in Ref. [302].

Usually, the $n_{\sigma}$ variable is the most used discriminating variable for PID and it is defined as the deviation of the measured signal from that expected for a given species $H_{i}$, in terms of the detector resolution $\sigma$. The minimum- $\sigma$ PID method is based on the deviation from the expected detector signal, but in this case only the species resulting in the smallest $n_{\sigma}$ value is chosen for each track, making this an exclusive selection. In case of the ALICE experiment, the TPC and TOF responses are combined by adding their respective $n_{\sigma}$ values in quadrature. This approach uses a similar logic to the Bayesian approach, but without requiring the input of any priors. Figure $43 \mathrm{a}$ is a clear example of the improvement of the $\Lambda_{\mathrm{c}}^{+} \rightarrow \mathrm{pK}^{-} \pi^{+}$identification in pp collisions at $\sqrt{s}=7 \mathrm{TeV}$ [302] when a Bayesian PID approach is used. The figure panel a shows the 

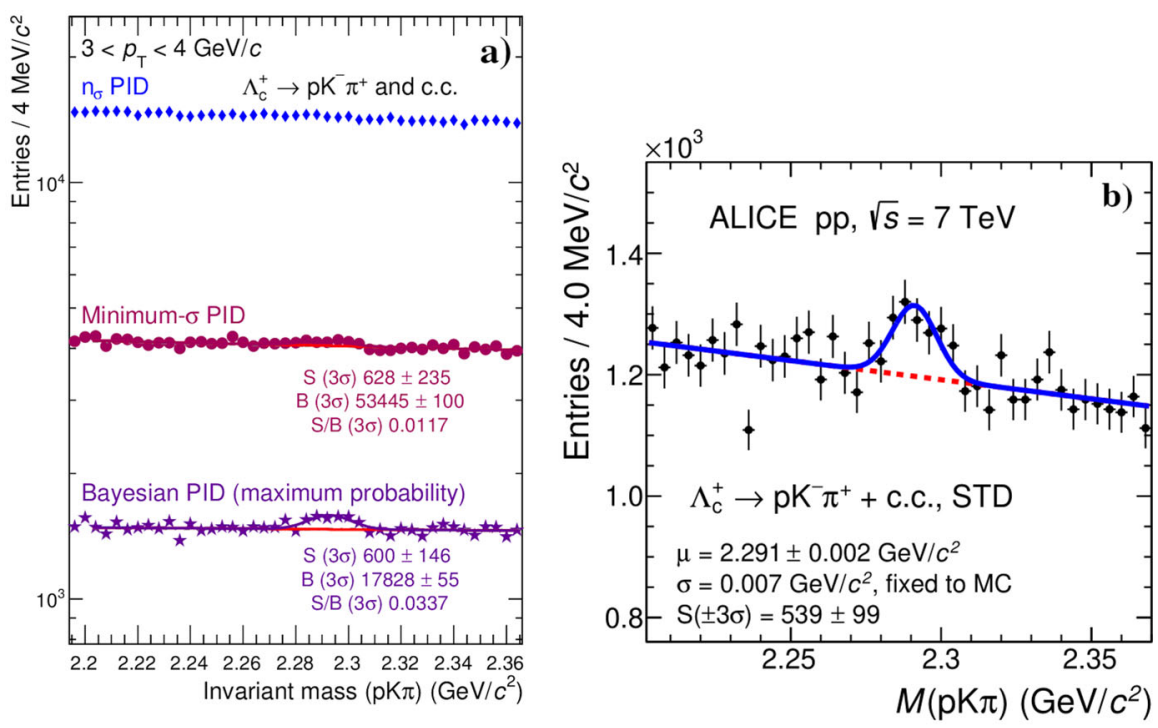

Fig. 43 ALICE. a Invariant mass spectra of $\Lambda_{\mathrm{C}}^{+}$in the range $3<p_{\mathrm{T}}<4 \mathrm{GeV} / c$, when $\pi, \mathrm{K}$ and $\mathrm{p}$ are identified using $n_{\sigma}$ PID, minimum- $\sigma$ PID and Bayesian PID in pp collisions at $\sqrt{s}=7 \mathrm{TeV}$, from [302]. b Invariant mass spectrum of $\Lambda_{\mathrm{c}}^{+}$in the range $3<p_{\mathrm{T}}<4 \mathrm{GeV} / c$ measured in pp collisions at $\sqrt{s}=7 \mathrm{TeV}$ when Bayesian PID method is used to identify $\pi, \mathrm{K}$ and p, from [315]

invariant mass spectra for $\mathrm{pK} \pi$ candidates in the $3<p_{\mathrm{T}}<4 \mathrm{GeV} / \mathrm{c}$ range obtained using the standard $n_{\sigma}$ PID approach, the alternative minimum- $\sigma$ PID and the Bayesian approach. The signal-to-background ratio improves significantly when the Bayesian approach is used. This PID method has been used in $\Lambda_{\mathrm{c}}^{+}$measurements in different systems [315]. The $\Lambda_{\mathrm{c}}^{+}$invariant mass distribution in $3<p_{\mathrm{T}}<4 \mathrm{GeV} / c$ range in pp collisions at $\sqrt{s}=7 \mathrm{TeV}$ is shown in Fig. $43 \mathrm{~b}$ as an example.

\section{Summary}

The present review described the latest trends in detector and analysis methods for particle and nuclei identification techniques, as currently adopted in nuclear physics experiments where INFN-CSN3 is involved. Although the basic physics processes underneath the identification are well established since several decades, the wide energy range to be covered by such experiments has pushed researchers to develop new hardware and software tools that profit of the new technological achievements, as described in these pages.

Table 2 summarizes the PID techniques presented in this review and the energy interval of application for each experiment. Few points emerged clearly: (i) the increasing importance of solid-state detectors (silicon or germanium), both for precise tracking and energy determination, (ii) the possibility to make a complete analysis of the signal shape, further strengthening the capabilities of techniques like PSA or timing, (iii) time resolutions of the order of 100 ps or below can now be achieved, (iv) the still intense use and R\&D on scintillators (liquids or crystals) and (v) the possibility to 


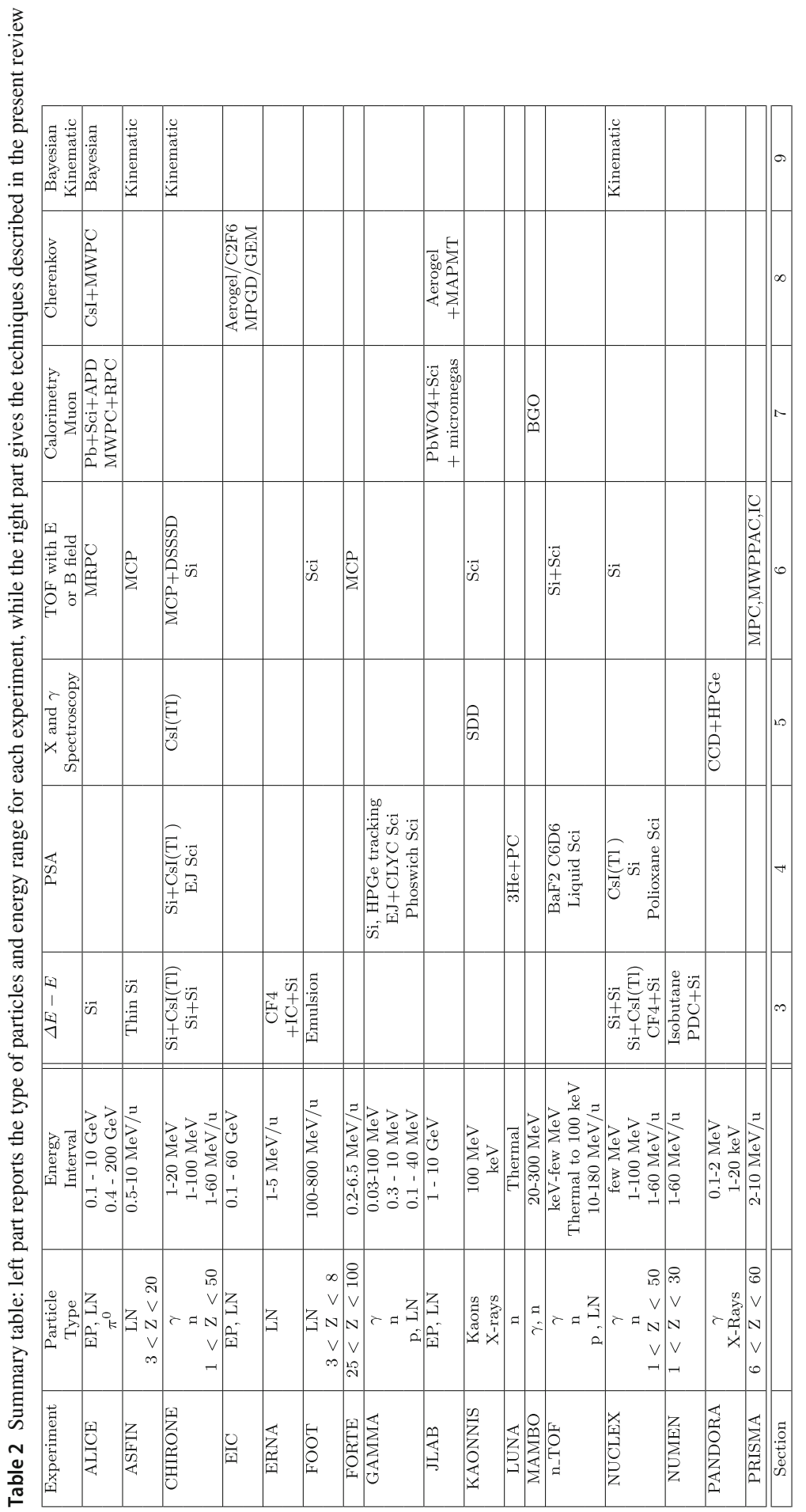

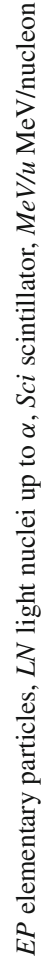


combine different techniques also profiting of the continuous increase of the data that can be collected.

Particle identification will remain a fundamental ingredient for all nuclear physics studies and there are still many opportunities for improvements in the techniques.

Acknowledgements The authors wish to thank P. Antonioli, A. Formicola, S. Palmerini, D. Santonocito, E. Scomparin for comments and suggestions to the draft. The authors wish to thank also the members of the INFN-CSN3 for continuous support and discussions. RD acknowledges funding from Italian Ministry of Education, University and Research (MIUR) through the "Dipartimenti di eccellenza" project Science of the Universe. CC acknowledges the support by the EU Horizon 2020 project AIDA-2020, GA no. 654168, the EU Horizon 2020 programme, STRONG-2020 project, GA no. 824093 and the Program Detector Generic R\&D for an Electron Ion Collider by Brookhaven National Laboratory, in association with Jefferson Lab and the DOE Office of Nuclear Physics. FC acknowledge the support from Gangneung-Wonju National University, Gangneung and Wonju, Korea.

Open Access This article is licensed under a Creative Commons Attribution 4.0 International License, which permits use, sharing, adaptation, distribution and reproduction in any medium or format, as long as you give appropriate credit to the original author(s) and the source, provide a link to the Creative Commons licence, and indicate if changes were made. The images or other third party material in this article are included in the article's Creative Commons licence, unless indicated otherwise in a credit line to the material. If material is not included in the article's Creative Commons licence and your intended use is not permitted by statutory regulation or exceeds the permitted use, you will need to obtain permission directly from the copyright holder. To view a copy of this licence, visit http://creativecommons.org/licenses/by/4.0/.

\section{References}

1. https://web.infn.it/csn3/index.php/it/

2. F. Gramegna et al., Il Nuovo Cimento C 42, 61 (2019). https://doi.org/10.1393/ncc/i2019-19061-6

3. https://www.lns.infn.it/it/potlns.html

4. P. Russotto et al., J. Phys. Conf. Ser. 1014, 012016 (2018). https://doi.org/10.1088/1742-6596/1014/ $1 / 012016$

5. C. Agodi et al., Universe 7, 72 (2021). https://doi.org/10.3390/universe7030072

6. P. Prati, J. Phys. Conf. Ser. 1342, 012088 (2020). https://doi.org/10.1088/1742-6596/1342/1/012088

7. A. Accardi et al., Eur. Phys. J. A 52, 268 (2016). https://doi.org/10.1140/epja/i2016-16268-9

8. NUPECC Long range plan 2017 https://www.esf.org/fileadmin/user_upload/esf/Nupecc-LRP2017. pdf

9. C. Broggini et al., La Rivista del Nuovo Cimento 42, 103 (2019). https://doi.org/10.1393/ncr/i201910157-1

10. R.L. Jaffe, A. Manohar, Nucl. Phys. B 337, 509(1990). https://doi.org/10.1016/0550-3213(90)905069

11. F. Gelis et al., Ann. Rev. Nucl. Part. Sci. 60, 46 (2010). https://doi.org/10.1146/annurev.nucl.010909. 083629

12. C.A. Aidala et al., Rev. Mod. Phys. 85, 655 (2013). https://doi.org/10.1103/RevModPhys.85.655

13. X.-D. Ji, Phys. Rev. D 55, 7114 (1997). https://doi.org/10.1103/PhysRevD.55.7114

14. X.-D. Ji, Phys. Rev. Lett. 78, 610 (1997). https://doi.org/10.1103/PhysRevLett.78.610

15. National Academies of Sciences, Engineering, and Medicine, "An Assessment of U.S.-Based Electron-Ion Collider Science." The National Academies Press, Washington DC, 2018. https://doi. org/10.17226/25171

16. A. Aprahamian et al., DOE/NSF Nuclear Science Advisory Panel (NSAC) Report. https://www.osti. gov/biblio/1296778 (2015)

17. E.C. Aschenauer et al., Phys. Rev. D 92, 094030 (2015). https://doi.org/10.1103/PhysRevD.92. 094030

18. E.-C. Aschenauer et al., J. High Energy Phys. 09, 093 (2013). https://doi.org/10.1007/ JHEP09(2013)093

19. L. Zheng et al., Phys. Rev. D 98, 034011 (2018). https://doi.org/10.1103/PhysRevD.98.034011 
20. M. Arratia et al., Phys. Rev. C 101, 065204 (2020). https://doi.org/10.1103/PhysRevC.101.065204

21. X. Liu et al., Phys. Rev. Lett. 122, 192003 (2019). https://doi.org/10.1103/PhysRevLett.122.192003

22. V.D. Burkert et al., Nucl. Instrum. Methods A 959, 163419 (2020)

23. J. Dudek et al., Eur. Phys. J. A 48, 187 (2012)

24. H. Avakian et al., arXiv:1202.1910v2 [hep-ex] (2012)

25. K. Hicks et al., APS Division Nuclear Physics Hawaii Meeting 2019, abstract id.FH.002. https:// meetings.aps.org/Meeting/DNP19/Session/FH.2

26. C. Curceanu et al., Symmetry 12, 547 (2020). https://doi.org/10.3390/sym12040547

27. C. Curceanu et al., Rev. Mod. Phys. 91, 0255006 (2019). https://doi.org/10.1103/RevModPhys.91. 025006

28. C. Curceanu et al., Condens. Matter. 4(2), 42 (2019). https://doi.org/10.3390/condmat4020042

29. M. Miliucci et al., Condens. Matter 4(1), 31 (2019). https://doi.org/10.3390/condmat4010031

30. C. Milardi et al., Int. J. Mod. Phys. A 24, 360 (2009). https://doi.org/10.1142/S0217751X09043687

31. M. Zobov et al., Phys. Rev. Lett. 104, 174801 (2010). https://doi.org/10.1103/PhysRevLett.104. 174801

32. M. Bazzi et al. (SIDDHARTA collaboration), Phys. Lett. B 704, 113 (2011). https://doi.org/10.1016/ j.physletb.2011.09.011

33. M. Bazzi et al. (SIDDHARTA collaboration), Nucl. Phys. A 881, 88 (2012). https://doi.org/10.1016/ j.nuclphysa.2011.12.008

34. M. Bazzi et al. (SIDDHARTA collaboration), Phys. Lett. B 681, 310 (2009). https://doi.org/10.1016/ j.physletb.2009.10.052

35. M. Bazzi et al. (SIDDHARTA collaboration), Phys. Lett. B 697, 199 (2011). https://doi.org/10.1016/ j.physletb.2011.02.001

36. D. Sirghi et al., J. Phys. Conf. Ser. 1526, 012023 (2020). https://doi.org/10.1088/1742-6596/1526/ $1 / 012023$

37. S. Alef et al., Eur. Phys. J. A 56, 104 (2020). https://doi.org/10.1140/epja/s10050-020-00107-X

38. S. Alef et al., Eur. Phys. J. A 57, 80 (2021). https://doi.org/10.1140/epja/s10050-021-00392-0

39. T. Jude et al., Phys. Lett. B 820, 136559 (2021). https://doi.org/10.1016/j.physletb.2021.136559

40. K. Aamodt et al. [ALICE], J. Instrum. 3, S08002 (2008). https://doi.org/10.1088/1748-0221/3/08/ S08002

41. Abelev et al. [ALICE], Int. J. Mod. Phys. A 29, 1430044 (2014). https://doi.org/10.1142/ S0217751X14300440

42. L. Cifarelli et al., Nuovo Cimento 10, 497 (2016). https://doi.org/10.1393/ncr/i2016-10128-0

43. A. Kalweit et al., Nucl. Phys. A 982, 1 (2019). https://doi.org/10.1016/j.nuclphysa.2018.10.015

44. A. Pagano, Nucl. Phys. News 22(1), 25 (2012). https://doi.org/10.1080/10619127.2011.629922

45. E. De Filippo, A. Pagano, Eur. Phys. J. A 50, 32 (2014). https://doi.org/10.1140/epja/i2014-14032-y

46. P. Russotto et al., Eur. Phys. J. A 56, 12 (2020). https://doi.org/10.1140/epja/s10050-019-00011-z

47. P. Russotto et al., Phys. Rev. C 94, 034608 (2016). https://doi.org/10.1103/PhysRevC.94.034608

48. J. Bishop et al., Phys. Rev. C 100, 034320 (2019). https://doi.org/10.1103/PhysRevC.100.034320

49. E. De Filippo et al., in preparation EPJA

50. A. Di Nitto et al., Phys. Rev. C 93, 044602 (2016). https://doi.org/10.1103/PhysRevC.93.044602

51. A. Di Nitto et al., Nucl. Phys. A 971, 21 (2018). https://doi.org/10.1016/j.nuclphysa.2018.01.008

52. A. Di Nitto et al., Phys. Rev. C 102, 024624 (2020). https://doi.org/10.1103/PhysRevC.102.024624

53. E. Vardaci et al., Eur. Phys. J. A 57, 95 (2021). https://doi.org/10.1140/epja/s10050-021-00400-3

54. E. Vardaci et al., Letter of Intent presented at LNL (2016). https://agenda.infn.it/event/10539/ overview

55. T. Marchi et al., J. Phys. Conf. Ser. 1643, 012036 (2020). https://doi.org/10.1088/1742-6596/1643/ $1 / 012036$

56. E. Vardaci et al., J. Phys. G Nucl. Part. Phys. 46, 115111 (2019). https://doi.org/10.1088/1361-6471/ ab445a

57. A. Di Nitto et al., Phys. Lett. B 784, 199 (2018). https://doi.org/10.1016/j.physletb.2018.07.058

58. K.V. Novikov et al., Phys. Rev. C 102, 044605 (2020). https://doi.org/10.1103/PhysRevC.102.044605

59. E. Vardaci et al., Phys. Rev. C 101, 064612 (2020). https://doi.org/10.1103/PhysRevC.101.064612

60. W. Korten et al., Eur. Phys. J. A 56, 137 (2020). https://doi.org/10.1140/epja/s10050-020-00132-W

61. W. Korten et al., Eur. Phys. J. A 56, 5 (2020). https://doi.org/10.1140/epja/s10050-020-00132-w

62. S. Valdré et al., Phys. Rev. C 93, 034617 (2016). https://doi.org/10.1103/PhysRevC.93.034617

63. L. Morelli et al., Phys. Rev. C 99, 054610 (2019). https://doi.org/10.1103/PhysRevC.99.054610 
64. A. Camaiani et al., Phys. Rev. C 97, 044607 (2018). https://doi.org/10.1103/PhysRevC.97.044607

65. S. Piantelli et al., Phys. Rev. C 96, 034622 (2017). https://doi.org/10.1103/PhysRevC.96.034622

66. A. Camaiani et al., Phys. Rev. C 103, 014605 (2021). https://doi.org/10.1103/PhysRevC.103.014605

67. S. Piantelli et al. (FAZIA Collaboration), Phys. Rev. C 103, 014603 (2021). https://doi.org/10.1103/ PhysRevC.103.014603

68. S. Barlini et al., Phys. Rev. C 87, 054607 (2013). https://doi.org/10.1103/PhysRevC.87.054607

69. R. Bougault et al., Eur. Phys. J. A 50, 47 (2014). https://doi.org/10.1016/j.nima.2019.03.082

70. S. Valdré et al., Nucl. Instrum. Methods A 930, 27 (2019). https://doi.org/10.1016/j.nima.2019.03. 082

71. M. Bruno et al., Eur. Phys. J. A 49, 128 (2013). https://doi.org/10.1140/epja/i2013-13128-2

72. D. Dell'Aquila et al., Nucl. Instrum. Methods A 877, 227 (2018). https://doi.org/10.1016/j.nima. 2017.09.046

73. T. Roger et al., Nucl. Instrum. Methods A 895, 126 (2018). https://doi.org/10.1016/j.nima.2018.04. 003

74. F. Cappuzzello et al., Eur. Phys. J. A 54, 72 (2018). https://doi.org/10.1140/epja/i2018-12509-3

75. E. Santopinto et al., Phys. Rev. C 98, 061601(R) (2018). https://doi.org/10.1103/PhysRevC.98. 061601

76. J.I. Bellone et al., Phys. Lett. B 807, 135528 (2020). https://doi.org/10.1016/j.physletb.2020.135528

77. H. Lenske et al., Prog. Part. Nucl. Phys. 109, 103716 (2019). https://doi.org/10.1016/j.ppnp.2019. 103716

78. F. Cappuzzello et al., Eur. Phys. J. A 51, 145 (2015). https://doi.org/10.1140/epja/i2015-15145-5

79. F. Cappuzzello et al., Eur. Phys. J. A 52, 167 (2016). https://doi.org/10.1140/epja/i2016-16167-1

80. F. Cappuzzello, D. Carbone, M. Cavallaro, Nucl. Instrum. Methods A 638, 74 (2011). https://doi.org/ 10.1016/j.nima.2011.02.045

81. J.R.B. Oliveira et al., J. Phys. G 40, 105101 (2013). https://doi.org/10.1088/0954-3899/40/10/105101

82. D. Carbone et al., Phys. Rev. C 90, 064621 (2014). https://doi.org/10.1103/PhysRevC.90.064621

83. F. Cappuzzello et al., Nat. Commun. 6, 6743 (2015). https://doi.org/10.1038/ncomms 7743

84. V. Soukeras et al., Phys. Rev. C 91, 057601 (2015). https://doi.org/10.1103/PhysRevC.91.057601

85. B. Paes et al., Phys. Rev. C 96, 044612 (2017). https://doi.org/10.1103/PhysRevC.96.044612

86. M.J. Ermamatov et al., Phys. Rev. C 96, 044603 (2017). https://doi.org/10.1103/PhysRevC.96.044603

87. D. Carbone et al., Phys. Rev. C 102, 044606 (2020). https://doi.org/10.1103/PhysRevC.102.044606

88. A. Spatafora et al., Phys. Rev. C 100, 034620 (2019). https://doi.org/10.1103/PhysRevC.100.034620

89. M. Cavallaro et al., Res. Phys. 13, 102191 (2019). https://doi.org/10.1016/j.rinp.2019.102191

90. L. Corradi et al., J. Phys. G Nucl. Part. Phys. 36, 113101 (2009). https://doi.org/10.1088/0954-3899/ $36 / 11 / 113101$

91. F. Galtarossa et al., Phys. Rev. C 97, 054606 (2018). https://doi.org/10.1103/PhysRevC.97.054606

92. D. Montanari et al., Phys. Rev. Lett. 113, 052501 (2014). https://doi.org/10.1103/PhysRevLett.113. 052501

93. L. Corradi et al., Nucl. Instrum. Methods B 317, 743 (2013). https://doi.org/10.1016/j.nimb.2013.04. 093

94. S. Szilner et al., Phys. Rev. C 76, 024604 (2007). https://doi.org/10.1103/PhysRevC.76.024604

95. D. Montanari et al., Phys. Lett. B 697, 288 (2011). https://doi.org/10.1016/j.physletb.2011.01.046

96. N. Mărginean et al., Phys. Lett. B 633, 696 (2006). https://doi.org/10.1016/j.physletb.2005.12.047

97. E. Sahin et al., Phys. Rev. C 91, 034302 (2015). https://doi.org/10.1103/PhysRevC.91.034302

98. R.E. Tribble et al., Rep. Prog. Phys. 77, 106901 (2014). https://doi.org/10.1088/0034-4885/77/10/ 106901

99. C. Spitaleri et al., Eur. Phys. J. A 55, 161 (2019). https://doi.org/10.1140/epja/i2019-12833-0

100. G.L. Guardo et al., Phys. Rev. C 95, 025807 (2017). https://doi.org/10.1103/PhysRevC.95.025807

101. M. La Cognata et al., Astrophys. J. 65, 846 (2017). https://doi.org/10.3847/1538-4357/aa845f

102. L. Lamia et al., Astrophys. J. 879, 23 (2019). https://doi.org/10.3847/1538-4357/ab2234

103. D. Jelavić Malenica et al., Phys. Rev. C 99, 064318 (2019). https://doi.org/10.1103/PhysRevC.99. 064318

104. R.G. Pizzone et al., Eur. Phys. J. A 52, 24 (2016). https://doi.org/10.1140/epja/i2016-16024-3

105. S.E. Woosley et al., Astrophys. J. 607, 921 (2004). https://doi.org/10.1086/383530

106. I. Baraffe et al., Astrophys. J. 615, 378 (2004). https://doi.org/10.1086/423982

107. C. Iliadis, Nuclear Physics of Stars (Wiley-VCH Verlag GmbH \& Co., Weinheim, 2015). https://doi. org/10.1002/9783527618750 
108. F. Strieder, C. Rolfs, Prog. Part. Nucl. Phys. 59, 562 (2007). https://doi.org/10.1016/j.ppnp.2007.02. 001

109. L. Barrón-Palos et al., Nucl. Phys. A 779, 318 (2006). https://doi.org/10.1016/j.nuclphysa.2006.09. 004

110. C. Angulo, Lect. Note Phys. 764, 253 (2009). https://doi.org/10.1007/978-3-540-85839-3_7

111. A. Best et al., Nucl. Instrum. Methods A (2016). https://doi.org/10.1016/j.nima.2015.12.034

112. A. Boeltzig et al., Phys. Lett. B 795, 122 (2019). https://doi.org/10.1016/j.physletb.2019.05.044

113. S. Cristallo et al., Astrophys. J. 859, 105 (2018). https://doi.org/10.3847/1538-4357/aac177

114. G.F. Ciani et al., Eur. Phys. J. A 56, 75 (2020). https://doi.org/10.1140/epja/s10050-020-00077-0

115. L. Csedreki et al., Nucl. Instrum. Methods A (2021). https://doi.org/10.1016/j.nima.2021.165081

116. J. Balibrea-Correa et al., Nucl. Instrum. Methods A 906, 103 (2018). https://doi.org/10.1016/j.nima. 2018.07.086

117. C. Guerrero et al., Eur. Phys. J. A 49, 27 (2013). https://doi.org/10.1140/epja/i2013-13027-6

118. C. Weiss et al., Nucl. Instrum. Methods A 799, 90 (2015). https://doi.org/10.1016/j.nima.2015.07. 027

119. F. Gunsing et al., Eur. Phys. J. Plus 131, 371 (2016). https://doi.org/10.1140/epjp/i2016-16371-4

120. D. Mascali et al., Universe 8, 80 (2022). https://doi.org/10.3390/universe 8020080

121. D. Mascali et al., Eur. Phys. J. A 53, 145 (2017). https://doi.org/10.1140/epja/i2017-12335-1

122. E. Naselli et al., EPJ Web Conf. 227, 02006 (2020). https://doi.org/10.1051/epjconf/202022702006

123. Y. Litvinov et al., Rep. Progr. Phys. 74, 016301 (2011). https://doi.org/10.1088/0034-4885/74/1/ 016301

124. Takahashi et al., Phys. Rev. C 36, 1522 (1987). https://doi.org/10.1103/PhysRevC.36.1522

125. F. Bosch et al., Phys. Rev. Lett. 77, 5190 (1996). https://doi.org/10.1103/PhysRevLett.77.5190

126. M. Jung et al., Phys. Rev. Lett. 69, 2164 (1992). https://doi.org/10.1103/PhysRevLett.69.2164

127. G. Battistoni et al., Front. Phys. (2021). https://doi.org/10.3389/fphy.2020.568242

128. P.A. Zyla et al. (Particle Data Group), Prog. Theor. Exp. Phys. 2020, 083 C01 (2020). https://doi.org/ $10.1093 /$ ptep/ptaa104

129. N. Bohr, Phil. Mag. 25, 10 (1913). https://doi.org/10.1080/14786440108634305

130. J. Zickefoose et al., Phys. Rev. C 97, 065806 (2018). https://doi.org/10.1103/PhysRevC.97.065806

131. M. Romoli et al., Eur. Phys. J. A 54, 142 (2018). https://doi.org/10.1140/epja/i2018-12575-5

132. Canberra Industries Inc., Pips Silicon Detectors Catalogue. http://www.canberra.com/products/ detectors/pips-detectors-single-multiple.asp

133. L. Morales-Gallegos et al., Eur. Phys. J. A (submitted)

134. L. Morales-Gallegos et al., Eur. Phys. J. A 54, 132 (2018). https://doi.org/10.1140/epja/i2018-125648

135. P. Figuera, PoS X LASNPA (2013). https://doi.org/10.22323/1.194.0089

136. G. Thungstrom et al., Nucl. Instrum. Methods A 391, 315 (1997). https://doi.org/10.1016/S01689002(97)00408-7

137. F. Amorini et al., Nucl. Instrum. Methods A 550, 248 (2005). https://doi.org/10.1016/j.nima.2005. 04.070

138. G. Cardella et al., Nucl. Instrum. Methods A 378, 262 (1996). https://doi.org/10.1016/01689002(96)00164-7

139. A. Di Pietro et al., Phys. Atom. Nucl. 69, 1366 (2008). https://doi.org/10.1134/S106377880608014X

140. F. Amorini et al., Nucl. Instrum. Methods A 834, 758 (2010). https://doi.org/10.1016/j.nuclphysa. 2010.01 .138

141. S. Tudisco et al., Sensors 18, 2289 (2018). https://doi.org/10.3390/s18072289

142. S. Tudisco et al., WO/2020/012288, PCT/IB2019/055491 (2019)

143. L. Torrisi, A. Cannavò, IEEE Trans. Electron. Dev. 63, 4445 (2016). https://doi.org/10.1109/ted.2016. 2612237

144. N.S. Martorana et al., Phys. Lett. B 782, 112 (2018). https://doi.org/10.1016/j.physletb.2018.05.019

145. M. Cavallaro et al., Eur. Phys. J. A 48, 59 (2012). https://doi.org/10.1140/epja/i2012-12059-8

146. D. Torresi et al., Nucl. Instrum. Methods A 989, 164918 (2021). https://doi.org/10.1016/j.nima.2020. 164918

147. A. Cunsolo et al., Nucl. Instrum. Methods A 484, 56 (2002). https://doi.org/10.1016/S01689002(01)02004-6

148. F. Cappuzzello et al., Nucl. Instrum. Methods A 621, 419 (2010). https://doi.org/10.1016/j.nima. 2010.05.027 
149. M. Cavallaro et al., Nucl. Instrum. Methods B 463, 334 (2020). https://doi.org/10.1016/j.nimb.2019. 04.069

150. S. Calabrese et al., Nucl. Instrum. Methods A 980, 164500 (2020). https://doi.org/10.1016/j.nima. 2020.164500

151. B. Alessandro et al., J. Instrum. 5, P02008 (2010). https://doi.org/10.1088/1748-0221/5/02/P02008

152. J. Adam et al. [ALICE], Phys. Lett. B 752, 267 (2016). https://doi.org/10.1016/j.physletb.2015.11. 048

153. J. Alme et al. [ALICE], Nucl. Instrum. Methods A 622, 316 (2010). https://doi.org/10.1016/j.nima. 2010.04 .042

154. F. Reidt et al. [ALICE], Nucl. Phys. A 1005, 121793 (2021). https://doi.org/10.1016/j.nuclphysa. 2020.121793

155. G. Galati et al., submitted to Open Physics

156. M.C. Montesi et al., Open Phys. 17, 233 (2019). https://doi.org/10.1515/phys-2019-0024

157. M. Alderighi et al., Nucl. Instrum. Methods A 489, 257 (2002). https://doi.org/10.1016/S01689002(02)00800-8

158. E. De Filippo et al., J. Phys. Conf. Ser 1014, 012003 (2018). https://doi.org/10.1088/1742-6596/ $1014 / 1 / 012003$

159. F. Amorini et al., IEEE Trans. Nucl. Sci. 59, 1772 (2012). https://doi.org/10.1109/TNS.2012.2201499

160. G. Pasquali et al., Nucl. Instrum. Methods A 570, 126 (2007). https://doi.org/10.1016/j.nima.2006. 10.008

161. C. Frosin et al., Nucl. Instrum. Methods A 951, 163018 (2020). https://doi.org/10.1016/j.nima.2019. 163018

162. J. England, G. Field, T. Ophel, Nucl. Instrum. Methods A 280, 291 (1989). https://doi.org/10.1016/ 0168-9002(89)90920-0

163. G. Pausch et al., Nucl. Instrum. Methods A 322, 43 (1992). https://doi.org/10.1016/01689002(92)90356-9

164. M. Alderighi et al., IEEE Trans. Nucl. Sci. 52, 1624 (2005). https://doi.org/10.1109/TNS.2005. 856991

165. M. Pârlog, H. Hamrita, B. Borderie, Nucl. Instrum. Methods A 613, 290 (2010). https://doi.org/10. 1016/j.nima.2009.12.010

166. L. Bardelli et al., Nucl. Instrum. Methods A 654, 272 (2011). https://doi.org/10.1016/j.nima.2011. 06.063

167. P.A. Tove, W. Seibt, Nucl. Instrum. Methods 51, 261 (1967). https://doi.org/10.1016/0029$554 X(67) 90012-2$

168. A.A. Quaranta, A. Taroni, G. Zanarini, IEEE Trans. Nucl. Sci. 15, 373 (1968). https://doi.org/10. 1109/TNS.1968.4324961

169. S. Pirrone et al., Eur. Phys. J. A 55, 22 (2019). https://doi.org/10.1140/epja/i2019-12695-4

170. P. Russotto et al., Phys. Rev. C 91, 014610 (2015). https://doi.org/10.1103/PhysRevC.91.014610

171. D. Mengoni et al., Nucl. Instrum. Methods A 764, 241 (2014). https://doi.org/10.1016/j.nima.2014. 07.054

172. Cieplicka-Oryńczak et al., Eur. Phys. J. A 54, 209 (2018). https://doi.org/10.1140/epja/i2018-126449

173. S. Capra et al., IEEE Trans. Nucl. Sci. 67, 1877 (2020). https://doi.org/10.1109/TNS.2020.3006892

174. A. Pullia et al., Rev. Sci. Instrum. 89, 026107 (2018). https://doi.org/10.1063/1.5012081

175. S. Capra et al., Nucl. Instrum. Methods A 935, 178 (2019). https://doi.org/10.1016/j.nima.2019.05. 039

176. S. Capra, IEEE Trans. Nucl. Sci. 67, 722 (2020). https://doi.org/10.1109/TNS.2020.2975311

177. F.C.L. Crespi et al., Nucl. Instrum. Methods A 602, 520 (2009). https://doi.org/10.1016/j.nima.2009. 01.101

178. J.A. Dueñas et al., Nucl. Instrum. Methods A 743, 44 (2014). https://doi.org/10.1016/j.nima.2014. 01.009

179. M. Assié et al., Eur. Phys. J. A 51, 11 (2015). https://doi.org/10.1140/epja/i2015-15011-6

180. R.J. Aliaga et al., 2015 IEEE Nucl. Sci. Conf. R and Med. Imaging, NSS/MIC 2015, 7581779 (2016). https://doi.org/10.1109/NSSMIC.2015.7581779

181. R.J. Aliaga et al., Nucl. Instrum. Methods A 800, 34 (2015). https://doi.org/10.1016/j.nima.2015.07. 067

182. Z. Sosin, Nucl. Instrum. Methods A 693, 170 (2012). https://doi.org/10.1016/j.nima.2012.07.020 
183. N. Le Neindre et al., Nucl. Instrum. Methods A 701, 145 (2013). https://doi.org/10.1016/j.nima.2012. 11.005

184. G. Pastore et al., Nucl. Instrum. Methods A 860, 42 (2017). https://doi.org/10.1016/j.nima.2017.01. 048

185. E.V. Pagano et al., Nucl. Instrum. Methods A 889, 83 (2018). https://doi.org/10.1016/j.nima.2018. 02.010

186. E.V. Pagano et al., Nucl. Instrum. Methods A 905, 47 (2018). https://doi.org/10.1016/j.nima.2018. 07.034

187. E.C. Pollacco et al., Nucl. Instrum. Methods A 887, 81 (2018). https://doi.org/10.1016/j.nima.2018. 01.020

188. F. Camera, A. Maj PARIS White Book, Krakow (2021). https://rifj.ifj.edu.pl/bitstream/handle/item/ 333/PARIS_WB.pdf

189. J.J. Valiente Dobón et al., Nucl. Instrum. Methods A 927, 81 (2019). https://doi.org/10.1016/j.nima. 2019.02.021

190. A. Giaz et al., Nucl. Instrum. Methods A 804, 212 (2015). https://doi.org/10.1016/j.nima.2015.09. 065

191. G. Hull et al., Nucl. Instrum. Methods A 925, 70 (2019). https://doi.org/10.1016/j.nima.2019.01.094

192. A. Giaz et al., Nucl. Instrum. Methods A 810, 132 (2016). https://doi.org/10.1016/j.nima.2015.11. 119

193. A. Giaz et al., Nucl. Instrum. Methods A 825, 51 (2016). https://doi.org/10.1016/j.nima.2016.03.090

194. L. Buonanno et al., Nuovo Cimento C 44, 30 (2021). https://doi.org/10.1393/ncc/i2021-21030-9

195. A. Giaz et al., Nucl. Instrum. Methods A 772, 103 (2015). https://doi.org/10.1016/j.nima.2014.10. 062

196. N. Blasi et al., Nucl. Instrum. Methods A 839, 23 (2016). https://doi.org/10.1016/j.nima.2016.09.039

197. L. Buonanno et al., 2020 IEEE International Conference on Artificial Intelligence Circuits and Systems. AICAS 2020. 9073914, 168 (2020). https://doi.org/10.1109/AICAS48895.2020.9073914

198. Z.M. Zeng et al., Nucl. Instrum. Methods A 866, 242 (2017). https://doi.org/10.1016/j.nima.2017. 04.009

199. F. Ferraro et al., Front. Astron. Space Sci. 7, 119 (2021). https://doi.org/10.3389/fspas.2020.617946

200. C. Guerrero et al., Nucl. Instrum. Methods A 608, 424 (2009). https://doi.org/10.1016/j.nima.2009. 07.025

201. P.F. Mastinu et al., CERN-n_TOF-PUB-2013-002 (2013). https://cdsweb.cern.ch/record/1558147/ files/n_TOF-PUB-2013-002.pdf

202. P. Schillebeeckx et al., Nucl. Data Sheets 113, 3054 (2012). https://doi.org/10.1016/j.nds.2012.11. 005

203. M. Heil et al., Nucl. Instrum. Methods A 459, 229 (2001). https://doi.org/10.1016/S01689002(00)00993-1

204. K. Wisshak et al., Nucl. Instrum. Methods A 292, 595 (1990). https://doi.org/10.1016/01689002(90)90179-A

205. S. Marrone et al., Nucl. Instrum. Methods A 568, 904 (2006). https://doi.org/10.1016/j.nima.2006. 08.064

206. R. Plag et al., Nucl. Instrum. Methods A 496, 425 (2003). https://doi.org/10.1016/S01689002(02)01749-7

207. S. Marrone et al., Nucl. Instrum. Methods A 490, 299 (2002). https://doi.org/10.1016/S01689002(02)01063-X

208. T. Marchi et al., Sci. Rep. UK 9, 9154 (2019). https://doi.org/10.1038/s41598-019-45307-8

209. A. Bracco et al., Eur. Phys. J. A 51, 99 (2015). https://doi.org/10.1140/epja/i2015-15099-6

210. S. Akkoyun et al., Nucl. Instrum. Methods A 668, 26 (2012). https://doi.org/10.1016/j.nima.2011. 11.081

211. E. Clément et al., Nucl. Instrum. Methods Phys. Res. A 855, 1 (2017). https://doi.org/10.1016/j.nima. 2017.02.063

212. F. Zocca et al., IEEE Trans. Nucl. Sci. 55, 695 (2008). https://doi.org/10.1109/TNS.2008.918739

213. B. De Canditiis et al., Eur. Phys. J. A 56, 276 (2020). https://doi.org/10.1140/epja/s10050-02000287-6

214. F. Recchia et al., Nucl. Instrum. Methods A 604, 555 (2009). https://doi.org/10.1016/j.nima.2009. 02.042

215. M. Siciliano et al., Eur. Phys. J. A 57, 67 (2021). https://doi.org/10.1140/epja/s10050-021-00385-z 
216. P. Napiralla et al., Nucl. Instrum. Methods A 955, 163337 (2020). https://doi.org/10.1016/j.nima. 2019.163337

217. G. Cardella et al., Nucl. Instrum. Methods A 799, 64 (2015). https://doi.org/10.1016/j.nima.2015.07. 054

218. G. Cardella et al., J. Phys. Conf. Ser. 1668, 012004 (2020). https://doi.org/10.1088/1742-6596/1668/ $1 / 012004$

219. G. Cardella et al., Phys. Rev. C 104, 064315 (2021). https://link.aps.org/doi/10.1103/PhysRevC.104. 064315

220. A. Scordo et al., J. Anal. At. Spectrom. 35, 155 (2020). https://doi.org/10.1039/C9JA00269C

221. A. Scordo et al., Condens. Matter 4(2), 38 (2019). https://doi.org/10.3390/condmat4020038

222. A. Scordo et al., J. Instrum. C 13(4), 04002 (2018). https://doi.org/10.1088/1748-0221/13/04/C04002

223. E. Naselli et al., J. Instrum. C 14, 10008 (2019). https://doi.org/10.1088/1748-0221/14/10/C10008

224. D. Mascali et al., Rev. Sci. Instrum. 87, 02 A510 (2016). https://doi.org/10.1063/1.4939201

225. R. Racz et al., Plasma Sources Sci. Technol. 26, 075011 (2017). https://doi.org/10.1088/1361-6595/ aa758f

226. S. Biri et al., J. Instrum. P 16, 03003 (2021). https://doi.org/10.1088/1748-0221/16/03/P03003

227. E. Naselli et al., Condens. Matter 7, 5 (2022). https://doi.org/10.3390/condmat7010005

228. A. Goasduff et al., Nucl. Instrum. Methods A (2021). https://doi.org/10.1016/j.nima.2021.165753

229. A. Akindinov et al., Eur. Phys. J. Plus 128, 44 (2013). https://doi.org/10.1140/epjp/i2013-13044-X

230. G. Dellacasa et al., CERN-LHCC-2000-012 (2000). http://cds.cern.ch/record/430132

231. P. Cortese et al., CERN-LHCC-2002-016 (2002). http://cds.cern.ch/record/545834

232. A. Akindinov et al., Nucl. Instrum. Methods A 533, 74 (2004). https://doi.org/10.1016/j.nima.2004. 07.004

233. F. Carnesecchi et al., J. Instrum. C 14, 06023 (2019). https://doi.org/10.1088/1748-0221/14/06/ C06023

234. J. Adam et al. [ALICE], Eur. Phys. J. Plus 132, 99 (2017). https://doi.org/10.1140/epjp/i2017-112791

235. S. Acharya et al. [ALICE], Phys. Lett. B, 794, 50 (2019). https://doi.org/10.1016/j.physletb.2019.05. 028

236. A. Manna et al., EPJ Web Conf. 239, 01008 (2020). https://doi.org/10.1051/epjconf/202023901008

237. N. Terranova et al., EPJ Web Conf. 239, 01024 (2020). https://doi.org/10.1051/epjconf/ 202023901024

238. R.G. Pizzone et al., Eur. Phys. J. A 56, 283 (2020). https://doi.org/10.1140/epja/s10050-020-002858

239. V.Z. Goldberg et al., Phys. Rev. C 69, 024602 (2004). https://doi.org/10.1103/PhysRevC.69.024602

240. A. Di Pietro et al., J. Phys. Conf. Ser. 366, 012013 (2012). https://doi.org/10.1088/1742-6596/366/ $1 / 012013$

241. G. Ciavola et al., Nucl. Phys. A 701, 54c (2002). https://doi.org/10.1016/S0375-9474(01)01547-0

242. D. Torresi et al., Phys. Rev. C 96, 044317 (2017). https://doi.org/10.1103/PhysRevC.96.044317

243. A. Pagano et al., Nucl. Phys. A 734, 504 (2004). https://doi.org/10.1016/j.nuclphysa.2004.01.093

244. I. Lombardo et al., Nucl. Phys. B Proc. Suppl. 215, 272 (2011). https://doi.org/10.1016/j.nuclphysbps. 2011.04.028

245. G. Traini et al., Il Nuovo Cimento C 43, 16 (2020). https://doi.org/10.1393/ncc/i2020-20016-5

246. M. Morrocchi et al., Nucl. Instrum. Methods A 916, 116 (2019). https://doi.org/10.1016/j.nima.2018. 09.086

247. L. Galli et al., Nucl. Instrum. Methods A 953, 163146 (2020). https://doi.org/10.1016/j.nima.2019. 163146

248. W.S. Cho et al., J. High Energy Phys. 01, 26 (2016). https://doi.org/10.1007/JHEP01(2016)026

249. E.M. Kozulin et al., Instrum. Exp. Tech. 51, 44 (2008). https://doi.org/10.1134/S0020441208010041

250. M.D. Usang et al., Sci. Rep. 9, 1525 (2019). https://doi.org/10.1038/s41598-018-37993-7

251. S. Valdré et al., Il Nuovo Cimento C 43, 10 (2020). https://doi.org/10.1393/ncc/i2020-20010-y

252. A.M. Stefanini et al., Nucl. Phys. A 701,217c (2002). https://doi.org/10.1016/S0375-9474(01)015780

253. S. Beghini et al., Nucl. Instrum. Methods A 551, 364 (2005). https://doi.org/10.1016/j.nima.2005. 06.058

254. A. Latina et al., LNL-INFN(REP) 204/05, 205 (2004) 
255. G. Montagnoli et al., Nucl. Instrum. Methods A 547, 455 (2005). https://doi.org/10.1016/j.nima. 2005.03.158

256. T. Mijatovć, Study of heavy-ion reactions with large solid angle magnetic spectrometers. PhD Thesis, University of Zagreb (2015). https://urn.nsk.hr/urn:nbn:hr:217:870561

257. T. Mijatové et al., Eur. Phys. J. A 52, 113 (2016). https://doi.org/10.1140/epja/i2016-16113-3

258. E. Fioretto et al., Nucl. Instrum. Methods A 899, 73 (2018). https://doi.org/10.1016/j.nima.2018.05. 011

259. Y. Kharlov, PoS, LHCP2018, 231 (2018). https://doi.org/10.22323/1.321.0231

260. S. Acharya et al. [ALICE], arXiv:2104.03116

261. G. Chabratova et al., ALICE-INT-2003-002. https://edms.cern.ch/document/371480/1

262. L. Aphecetche et al., ALICE-INT-2009-044. https://edms.cern.ch/document/1054937/1

263. J. Gaiser, Ph.D. Thesis, SLAC-R-255 (1982). https://inspirehep.net/literature/183554

264. J. Adam et al. [ALICE], CERN-LHCC-2015-001, ALICE-TDR-018 (2015). https://cds.cern.ch/ record/1981898? $\mathrm{ln}=\mathrm{it}$

265. A. Acker et al., Nucl. Instrum. Methods A 959, 163475 (2020). https://doi.org/10.1016/j.nima.2020. 163419

266. A. Acker et al., Nucl. Instrum. Methods A 957, 163423 (2020). https://doi.org/10.1016/j.nima.2020. 163423

267. D.S. Carman et al., Nucl. Instrum. Methods A 960, 163629 (2020). https://doi.org/10.1016/j.nima. 2020.163629

268. O. Chamberlain, E. Segre, C. Wiegand, T. Ypsilantis, Phys. Rev. 100, 947 (1955). https://doi.org/10. 1103/PhysRev.100.947

269. J. Seguinot, T. Ypsilantis, Nucl. Instrum. Methods 142, 377 (1977). https://doi.org/10.1016/0029554X(77)90671-1

270. S. Beole et al. [ALICE], CERN-LHCC-98-19. http://cds.cern.ch/record/381431

271. D. Di Bari [ALICE], Nucl. Instrum. Methods A 502, 300 (2003). https://doi.org/10.1016/S01689002(03)00292-4

272. Nucl. Instrum. Methods A, 876, 33 (2017)

273. X. He et al., Nucl. Instrum. Methods A 952, 162051 (2020). https://doi.org/10.1016/j.nima.2019.04. 005

274. X. He, mrich for eic-past present and future. https://indico.bnl.gov/event/7449/contributions/35910/ attachments/27157/41392/mRICH_YP_PID_Temple_v1.pdf, March 2020. Presentation at Temple Yellow Report Meeting

275. X. He, Description of detector and parameters. https://indico.bnl.gov/event/8419/contributions/ 37212/attachments/27855/42733/mRICH_fastParameterization.pdf, May 2020. Presentation at PID DWG Biweekly Meeting

276. X. He et al., mrich for eic yr. https://indico.bnl.gov/event/8419/contributions/37212/attachments/ 27855/42768/mRICH_YP_PID_May1_2020.pdf, May 2020. Presentation at PID DWG Biweekly Meeting

277. E. Cisbani et al., Dual ring imaging cherenkov status. https://indico.bnl.gov/event/7449/contributions/ 35908/attachments/27151/41432/EICYR-drich-200320.pdf, March 2020. Presentation at Temple Yellow Report Meeting

278. E. Cisbani et al., drich details for yr. https://indico.bnl.gov/event/8297/contributions/36712/ attachments/27616/42422/drich_toward_yr_v0.4.pdf, April 2020. Presentation at PID DWG Biweekly Meeting

279. L. Barion et al., J. Instrum. C 15, 02040 (2020). https://doi.org/10.1088/1748-0221/15/02/c02040

280. E. Cisbani et al., J. Instrum. P 15, 05009 (2020). https://doi.org/10.1088/1748-0221/15/05/p05009

281. E. Garutti et al., Nucl. Instrum. Methods A 926, 69 (2019). https://doi.org/10.1016/j.nima.2018.10. 191

282. R. Kugathasan, J. Instrum. C 15, 05019 (2020). https://doi.org/10.1088/1748-0221/15/05/c05019

283. M. Calvi et al., Nucl. Instrum. Methods A 922, 243 (2019). https://doi.org/10.1016/j.nima.2019.01. 013

284. M. Blatnik et al., IEEE Trans. Nucl. Sci. 62, 3256 (2015). https://doi.org/10.1109/TNS.2015.2487999

285. W. Anderson et al., Nucl. Instrum. Methods Phys. Res. A 646, 35 (2011). https://doi.org/10.1016/j. nima.2011.04.015

286. J. Agarwala et al., Nucl. Instrum. Methods A 952, 161832 (2020). https://doi.org/10.1016/j.nima. 2019.01.058 
287. J. Agarwala et al., Nucl. Instrum. Methods A 936, 416 (2019). https://doi.org/10.1088/1742-6596/ $1498 / 1 / 012007$

288. B. Azmoun et al., IEEE Trans. Nucl. Sci. 66, 1984 (2019). https://doi.org/10.1109/TNS.2019.2928269

289. J. Agarwala et al., J. Phys. Conf. Ser. 1498, 012007 (2020). https://doi.org/10.1088/1742-6596/1498/ $1 / 012007$

290. L. Velardi et al., Appl. Phys. Lett. 108, 083503 (2016). https://doi.org/10.1063/1.4942648

291. J. Agarwala et al., Nucl. Instrum. Methods A 952, 161967 (2020). https://doi.org/10.1016/j.nima. 2019.03.022

292. F.M. Brunbauer et al., J. Instrum. C 15, 09052 (2020). https://doi.org/10.1088/1748-0221/15/09/ c09052

293. S. Dalla Torre, High momentum PID at EIC (in 10 years from now). https://indico.bnl.gov/ event/7449/contributions/35912/attachments/27095/41303/DallaTorre_RICH_high-p_March2020_ Temple.pdf. Presentation at the 1st EIC Yellow Report Workshop at Temple University, 19-21 March 2020

294. G. Volpe, Focusing RICH for high-momentum PID: preliminary results from simulation. https:// indico.bnl.gov/event/9062/contributions/40118/attachments/29711/46342/YR_PIDmeeting_31_ 07_2020.pdf. Presentation at PID DWG Biweekly Meeting

295. AYu. Barnyakov et al., Nucl. Instrum. Methods A 453, 326 (2000). https://doi.org/10.1016/S01689002(00)00653-7

296. M. Contalbrigo et al., Nucl. Instrum. Methods A 964, 163791 (2020). https://doi.org/10.1016/j.nima. 2020.163791

297. G.J. Barber et al., Nucl. Instrum. Methods A 593, 624 (2008). https://doi.org/10.1016/j.nima.2008. 05.050

298. R. Canestrari et al., Proc. SPIE 7437, 743711 (2009). https://doi.org/10.1117/12.826145

299. V. Ziegler et al., Nucl. Instrum. Methods A 959, 163472 (2020). https://doi.org/10.1016/j.nima.2020. 163472

300. F. Carminati et al. [ALICE], J. Phys. G 30, 1517 (2004). https://doi.org/10.1088/0954-3899/30/11/ 001

301. B. Alessandro et al. [ALICE], J. Phys. G 32, 1295 (2006). https://doi.org/10.1088/0954-3899/32/10/ 001

302. J. Adam et al. [ALICE], Eur. Phys. J. Plus 131, 168 (2016). https://doi.org/10.1140/epjp/i201616168-5

303. E. Costanzo et al., Nucl. Instrum. Methods A 295, 373 (1990). https://doi.org/10.1016/01689002(90)90715-I

304. I. Indelicato et al., Astrophys. J. 845, 19 (2017). https://doi.org/10.3847/1538-4357/aa7de7

305. L. Acosta et al., Nucl. Instrum. Methods A 715, 56 (2013). https://doi.org/10.1016/j.nima.2013.03. 028

306. R.G. Markham et al., Nucl. Phys. A 270, 489 (1976). https://doi.org/10.1016/0375-9474(76)904589

307. D. Dell' Aquila et al., Phys. Rev. Lett. 119, 132501 (2017). https://doi.org/10.1103/PhysRevLett.119. 132501

308. L. Morelli et al., J. Phys. G Nucl. Part. Phys. 43, 045110 (2016). https://doi.org/10.1088/0954-3899/ $43 / 4 / 045110$

309. M. Bruno et al., J. Phys. G Nucl. Part. Phys. 46, 125101 (2019). https://doi.org/10.1088/1361-6471/ ab46cd

310. J. Adam et al. [ALICE], Phys. Lett. B 760, 720 (2016). https://doi.org/10.1016/j.physletb.2016.07. 050

311. J. Adam et al. [ALICE], Phys. Rev. C 93, 034913 (2016). https://doi.org/10.1103/PhysRevC.93. 034913

312. J. Adam et al. [ALICE], Eur. Phys. J. C 75, 226 (2015). https://doi.org/10.1140/epjc/s10052-015$3422-9$

313. B. Abelev et al. [ALICE], Phys. Lett. B 736, 196 (2014). https://doi.org/10.1016/j.physletb.2014.07. 011

314. P. Gregory, Bayesian Logical Data Analysis for the Physical Sciences (Cambridge University Press, 2005). https://doi.org/10.1017/CBO9780511791277

315. S. Acharya et al. [ALICE], J. High Energy Phys. 04, 108 (2018). https://doi.org/10.1007/ JHEP04(2018)108 


\section{Authors and Affiliations}

A. Badalà ${ }^{1}$ - M. La Cognata ${ }^{2}$ - R. Nania ${ }^{3}$ - M. Osipenko ${ }^{4} \cdot$ S. Piantelli ${ }^{5}$.

R. Turrisi ${ }^{6}$. L. Barion ${ }^{7}$. S. Capra ${ }^{8,9}$ - D. Carbone ${ }^{2}$. F. Carnesecchi ${ }^{3,26}$.

E. A. R. Casula 11,12 . C. Chatterjee ${ }^{13}$. G. F. Ciani ${ }^{14,25}$ - R. Depalo ${ }^{6,15}$.

A. Di Nitto ${ }^{16,17}$ - A. Fantini ${ }^{18,27}$. A. Goasduff ${ }^{19}$. G. L. Guardo ${ }^{2}$ A. C. $\operatorname{Kraan}^{20}$.

A. Manna ${ }^{3,10}$ - L. Marsicano ${ }^{4}$ N. S. Martorana ${ }^{2,21}$. L. Morales-Gallegos ${ }^{16}$.

E. Naselli' ${ }^{2,21}$ - A. Scordo 22 . S. Valdré ${ }^{5}$. G. Volpe 23,24

1 INFN-CT, Catania, Italy

2 INFN-LNS, Catania, Italy

3 INFN-BO, Bologna, Italy

4 INFN-GE, Genoa, Italy

5 INFN-FI, Florence, Italy

6 INFN-PD, Padua, Italy

7 INFN-FE, Ferrara, Italy

8 Università di Milano, Milan, Italy

9 INFN-MI, Milan, Italy

10 Dipartimento di Fisica e Astronomia, Università di Bologna, Bologna, Italy

11 INFN-CA, Cagliari, Italy

12 Dipartimento di Fisica, Università di Cagliari, Cagliari, Italy

13 INFN-TS, Trieste, Italy

14 Università degli Studi della Campania "L.Vanvitelli”, Caserta, Italy

15 Università degli Studi di Padova, Padua, Italy

16 INFN-NA, Naples, Italy

17 Dipartimento di Fisica "E. Pancini”, Università Federico II, Naples, Italy

18 Universitá degli Studi di Roma "Tor Vergata", Rome, Italy

19 INFN-LNL, Legnaro, PD, Italy

20 INFN-PI, Pisa, Italy

21 Dipartimento di Fisica e Astronomia "E. Majorana", Università di Catania, Catania, Italy

22 INFN-LNF, Frascati, RM, Italy

23 INFN-BA, Bari, Italy

24 Dipartimento Interateneo di Fisica “M. Merlin”, Bari, Italy

25 INFN-LNGS, L'Aquila, Italy

26 CERN, Geneva, Switzerland

27 INFN-Roma Tor Vergata, Rome, Italy 